\title{
Games and contests
}

Citation for published version (APA):

Cingiz, K. (2018). Games and contests. [Doctoral Thesis, Maastricht University]. Maastricht University. https://doi.org/10.26481/dis.20180704kc

Document status and date:

Published: 01/01/2018

DOI:

$10.26481 / \mathrm{dis} .20180704 \mathrm{kc}$

Document Version:

Publisher's PDF, also known as Version of record

\section{Please check the document version of this publication:}

- A submitted manuscript is the version of the article upon submission and before peer-review. There can be important differences between the submitted version and the official published version of record.

People interested in the research are advised to contact the author for the final version of the publication, or visit the DOI to the publisher's website.

- The final author version and the galley proof are versions of the publication after peer review.

- The final published version features the final layout of the paper including the volume, issue and page numbers.

Link to publication

\footnotetext{
General rights rights.

- You may freely distribute the URL identifying the publication in the public portal. please follow below link for the End User Agreement:

www.umlib.nl/taverne-license

Take down policy

If you believe that this document breaches copyright please contact us at:

repository@maastrichtuniversity.nl

providing details and we will investigate your claim.
}

Copyright and moral rights for the publications made accessible in the public portal are retained by the authors and/or other copyright owners and it is a condition of accessing publications that users recognise and abide by the legal requirements associated with these

- Users may download and print one copy of any publication from the public portal for the purpose of private study or research.

- You may not further distribute the material or use it for any profit-making activity or commercial gain

If the publication is distributed under the terms of Article $25 \mathrm{fa}$ of the Dutch Copyright Act, indicated by the "Taverne" license above, 


\section{Games and Contests}

Kutay Cingiz 
Games and Contests

(C) Kutay Cingiz, Maastricht 2018

All rights reserved. No part of this publication may be reproduced, stored in a retrieval system, or transmitted, in any form, or by any means, electronic, mechanical, photocopying, recording or otherwise, without the prior permission in writing from the author.

This book was typeset by the author using $\mathrm{HT}_{\mathrm{E}} \mathrm{X}$.

ISBN 9789462959606

Printed in the Netherlands by Datawyse 


\section{Games and Contests}

\section{DISSERTATION}

to obtain the degree of Doctor at Maastricht University, on the authority of the Rector Magnificus,

Prof.dr. Rianne M. Letschert

in accordance with the decision of the Board of Deans,

to be defended in public

on Wednesday 4 July 2018, at 10:00 hours

by

Kutay Cingiz 


\section{Supervisor:}

Prof. Dr. P. Jean-Jacques Herings

Co-Supervisor:

Dr. Arkadi Predtetchinski

Co-Supervisor:

Dr. János Flesch

Assessment Committee:

Prof. Dr. Frank Thuijsman (chairman)

Prof. Dr. Véronique Bruyère, Université de Mons, Belgium

Prof. Dr. M. Remzi Sanver, Université Paris-Dauphine, France

Dr. Christian Seel 


\section{Acknowledgements}

Don't Panic! This is my PhD thesis. And I had a wonderful time during my doctorate studies. I am surrounded with amazing people who supported me every step of the way. I would like to take this opportunity to rely my thanks to them.

First and foremost, I would like to thank my supervisors Jean-Jacques Herings, Arkadi Predtetchinski and Janos Flesch for their generously devoted time to our discussions, for their structured and efficient approach and for being very patient with me along the way. Their guidance, wisdom and immense knowledge helped me during research and writing of this thesis.

I want to thank Muhamet Yildız for making my research visit to MIT happen, and to Mehmet Ekmekçi for the lecture at Boston Collage and meetings.

I also would like to thank to my coauthors Mehmet İsmail and Nejat Anbarc 1 for a very collaborative and fruitful research.

Many thanks to Ali Nesin not just for providing us a Mathematics education with utmost quality but also teaching us the culture that comes with it. Thank you for allowing us to be part of the Nesin Math Village. I am proud to be a student of him. If Ali Nesin laid the foundation of my academic education then Remzi Sanver is the one who channelled it to Economics. His wisdom and foresightedness is the source of my choices that lead me to Maastricht eventually. 
I want to thank to my professors at Sabanci University, especially Özgür Kıbrıs for being an amazing master thesis supervisor and İzak Atiyas for the courses which I enjoyed very much.

This has not always been years with theorems and proofs but also traveling, music, friendship and family. I want to thank to Pink Floyd, AC/DC and Dire Straits for their marvelous music. I address my thanks to friends in Maastricht who are Murat Öztürk, Mehmet İsmail, Emre Ergin, Deniz Gürhan, Çağrı Öztürk, Sevim Şener, Seher Fazlığlu, Esra Altuntas, Hande Erkut, Burcu Duygu, Nordin Hanssen, Ronald Peeters, Diogo Geraldes, Jessie Lemmens and also to the superset of Turkish friends in Maastricht which is "The Turkish Community". I want to thank our friends and hosts Jeffrey Dines and Christina Lord at our journey in Boston. Go Panthers! I want to thank my friends Ceyhun Erdel, İbrahim Tezgel, Doruk Tunaoğlu, Mutlu Şen in Istanbul for not forgetting me and meeting me whenever I was in Istanbul.

And finally, I rely my absolute gratitude to my family. I would like to thank to my awesome parents, my mother Zeynep Cingiz, my father Hasan Cingiz who support me with all their heart. I would like to thank my brother Koray Ozan Cingiz for being the coolest brother ever. I want to thank to Ayse Muge Yuksel for 4 lovely years together.

I dedicate this book to my family. So long and thanks for all the fishes!

Kutay Cingiz

Maastricht, 2018 


\section{Contents}

Acknowledgements i

1 Introduction $\quad 1$

2 Doing It Now, Later, or Never 7

2.1 Introduction . . . . . . . . . . . . . . 7

2.2 The Procrastination Game . . . . . . . . . . . 10

2.3 The General Model . . . . . . . . . . . . . . . . . 13

2.4 Existence of Equilibrium in the Upper Semicontinuous Case 17

2.5 Existence of Naive $\epsilon$-Equilibrium . . . . . . . . . . . 21

2.6 Existence of Sophisticated $\epsilon$-Equilibrium . . . . . . . . . 22

2.7 Sophisticates Stop Earlier . . . . . . . . . . . 28

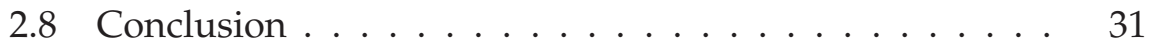

3 Perfect Information Games Where Each Player Acts Only Once 33

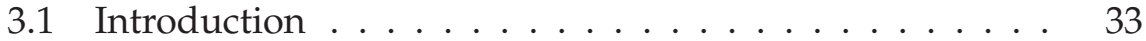

3.2 The model . . . . . . . . . . . . 36

3.3 Frequency-based minority games . . . . . . . . . 39

3.3.1 The leading example and the definition . . . . . 39

3.3.2 Further discussion on admissible sets . . . . . . 41

3.3.3 The main result and overview of the proof . . . 43

3.3.4 The proof of Theorem 3.3.4 . . . . . . . . . . . 44

3.4 Sufficient conditions for existence . . . . . . . . . 50 
3.4.1 Games with qualitative objectives . . . . . . . . 51

3.4.2 Games with upper semicontinuous payoffs . . . . 53

3.4.3 Games continuous outside a countable set . . . . 54

3.4.4 Games played by a finite number of teams . . . . 55

3.5 Two examples . . . . . . . . . . . . . 56

3.6 Conclusion . . . . . . . . . . . . . 60

4 Multi-Battle $n$-Player Dynamic Contests 63

4.1 Introduction . . . . . . . . . . . . 63

4.2 Model. . . . . . . . . . . . . . . 66

4.3 Maximizing the probability of winning . . . . . . 71

4.3.1 Example I: The dynamic contest with 3 identical states . . . . . . . . . . . . . 71

4.3.2 Example II: Dynamic contests with 4 states . . . . 74

4.4 Maximizing the expected number of delegates . . . . 77

4.5 Conclusion . . . . . . . . . . . . . . 80

$\begin{array}{ll}\text { Bibliography } & 91\end{array}$

$\begin{array}{ll}\text { Valorization } & 93\end{array}$

$\begin{array}{ll}\text { Biography } & 97\end{array}$ 


\section{Chapter 1}

\section{Introduction}

Non-cooperative game theory is a study of strategic interactions of rational and self-interested players where only self-enforcing coalitions are possible. One of the fundamental solution concepts of this branch of game theory is subgame perfect equilibrium. Reinhard Selten defined the concept of subgame perfect equilibrium in his 1965 paper Ein Oligopolmodell mit Nachfrageträgheit (An Oligopoly Model with Demand Inertia). This solution concept allows us to distinguish Nash equilibria with credible threats in perfect information games. A credible threat is expected to be carried out whereas a non-credible threat is not rational to be carried out.

Consider a two period sequential game with two players where each acts only once. First player 1 chooses between actions $A$ and $B$ then player 2 after observing the action decides on whether to play $C$ or $D$. The following Figure (1.1) provides the game tree. The Nash equilibria of the given game are $(A, D C)$ and $(A, D D)$. But from the sequential rationality assumption we know that if player 1 plays $B$ then player 2 never plays $D$. Hence the threat of player 2 to play $D$ if player 1 plays $B$ is non-credible. Thus the only subgame perfect equilibrium profile of the game is $(A, D C)$. 


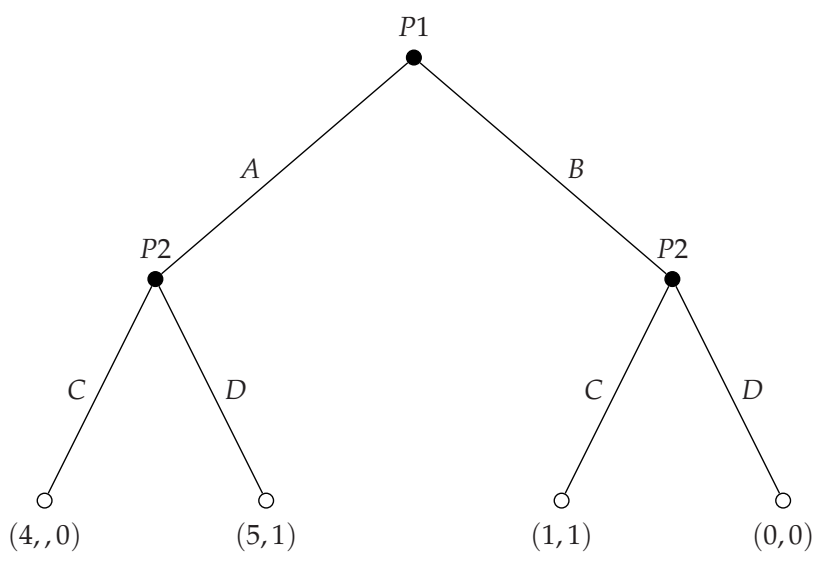

Figure 1.1: A game in extensive form.

Since Selten's introduction of subgame perfect equilibrium, there have been many variations on this solution concept one of which is the subgame perfect $\epsilon$-equilibrium. This solution concept is an approximation of subgame perfect equilibrium.

This book explores the existence of Selten's distinguished equilibrium concept and its approximation in certain classes of games. Moreover we analyze the existence of a subgame perfect equilibrium with proportional campaign resource allocation with respect to delegate numbers in certain classes of contests. The current dissertation consists of three chapters. The first two chapters focus on the class of games played by an infinite sequence of players. And the last chapter focuses on multi battle dynamic contests played by finite number of players.

\section{Outline of Chapters 2-4}

\section{Chapter 2}

In this chapter, we study centipede games played by an infinite sequence of players where each player acts only once. The active player at time 
$t$ chooses actions between stopping the game at time $t$ or to continue it. Consider the example in Figure (1.2) where the payoff for player $i \in \mathbb{N}$ is $1-1 /(t-i+1)$ if the game ends at time $t>i$, and 0 if the game ends at time $t \leq i$ or if no one stops. Following the literature on time-

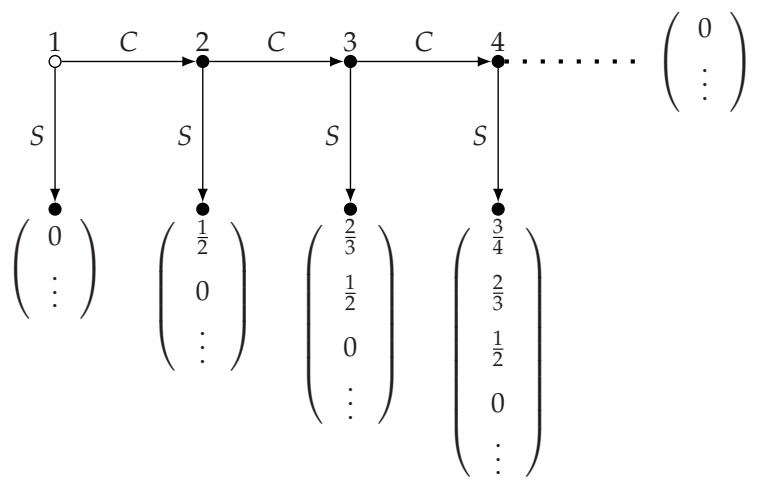

Figure 1.2: A game without naive equilibrium.

inconsistent preferences, we distinguish two types of decision makers, naive and sophisticated, and the corresponding solution concepts, naive $\epsilon$-equilibrium and sophisticated $\epsilon$-equilibrium. A naive decision maker erroneously believes that his current self controls all future decisions whereas a sophisticated decision maker knows that his current self only controls the decision at the current period but not the future periods.

For a naive decision maker, we prove that the infinite centipede game with uniformly bounded payoffs may not admit a naive 0 -equilibria. We then show that upper semicontinuity of payoffs is a sufficient condition for existence of naive 0 -equilibrium. We show the existence of naive $\epsilon$-equilibria for each positive $\epsilon$.

Regarding a sophisticated decision maker, we first show that sophisticated equilibrium does not always exist by giving a counter example. We prove that the infinite centipede game with uniformly bounded payoffs admits sophisticated 0-equilibrium whenever payoffs are upper semicon- 
tinuous. Finally, we show that there exists a sophisticated $\epsilon$-equilibrium, for $\epsilon>0$.

We also show that for every sophisticated $\epsilon$-equilibrium there exists a naive $\epsilon$-equilibrium and for every naive $\epsilon$-equilibrium there exists a sophisticated $\epsilon$-equilibrium such that the stopping probability of every player in the sophisticated $\epsilon$-equilibrium strategy is higher than in the naive $\epsilon$-equilibrium.

\section{Chapter 3}

In this chapter, we study perfect information games played by an infinite sequence of players, each acting only once in the course of the game. We introduce a class of frequency-based minority games where each player faces two actions. One action provides intermediate payoffs irrespective of what the other players do and the other action provides a high payoff whenever a minority of the players use it, otherwise a low payoff. We show that these games admit no subgame perfect $\epsilon$-equilibrium for small positive values of $\epsilon$.

Furthermore we derive a number of sufficient conditions to guarantee existence of subgame perfect $\epsilon$-equilibrium. These conditions are games with qualitative objectives (games where each player's payoff function takes either 0 or 1 ), games with upper semicontinuous payoffs, games continuous outside a countable set (games where the payoffs are continuous when restricted to a sufficiently large domain), and games played by a finite number of teams where each team consists of players with identical payoff functions.

\section{Chapter 4}

There are two main elections, U.S. presidential elections and U.S. presidential primaries, to determine the president of the United States. These elections (apart from their aim) differ by their voting system. Presiden- 
tial primaries is the method of how political parties in the United States decide on their strongest candidate to run for the presidential election. Each party holds a series of state-based elections-primaries - and candidates with the highest aggregate number of delegates throughout these elections become the parties' official nominees for the presidential run. Each party follows either a winner-take-all or proportional rule in each state-based election. The presidential primaries is an example of sequential multiple-battle dynamic contests whereas the presidential elections is an example of static (i.e., not dynamic) multi-battle contests.

Proportionality in campaign resource allocation with respect to delegate numbers is a desirable concept in presidential primaries. We introduce a model for $n$-player multi-battle dynamic contests to study proportionality. We show that there exists a subgame perfect equilibrium in which players allocate their resources proportionally when players maximize their expected number of delegates. However when players maximize their probability of winning, for dynamic contests with at least 4 states and at least 2 delegates, there is always a distribution of delegates over the states such that proportionality does not hold. 



\section{Chapter 2}

\section{Doing It Now, Later, or Never}

\subsection{Introduction}

We study centipede games played by an infinite sequence of players. Each player is active only once. The active player can choose either to stop the game or to continue. As soon as the active player chooses to stop, the game ends.

One of the main application areas of our model concerns the vast literature on decision making with time-inconsistent preferences. It is customary (Strotz (1955), Pollak (1968), Peleg and Yaari (1973), Goldman (1979)) to model a decision maker with time-inconsistent preferences as consisting of a sequence of multiple selves, where day $t$ self makes a decision on behalf of the decision maker on day $t$. This leads to a game played by an infinite sequence of players.

In their well-known paper Doing it now or later, O'Donoghue and Rabin (1999) consider a decision maker who has to decide when to execute a certain task. One important feature of their model is an exogenous deadline: once the deadline is reached, the decision maker has no choice but to execute the task. This model can be seen as a finite centipede game and is a special case of our more general model. In our model, the 
decision maker may have the option to never quit, whence the title of our chapter.

Following the literature on decision making with time-inconsistent preferences, we distinguish two types of decision makers, naive and sophisticated, and examine the corresponding two types of solution concepts.

A naive decision maker acts under the erroneous assumption that his current self controls all future decisions in the game. Thus a naive decision maker intends to follow a strategy that maximizes his payoff over the entire continuation game, but in reality he only carries out the first action. This happens because the strategy that is optimal for the current self need not be optimal for future selves. This behavior is captured by the concept of naive equilibrium.

A sophisticated decision maker, in contrast, is fully aware that his day $t$ self only controls the decision on day $t$, and that the future selves have different preferences. Thus in a sophisticated equilibrium each self of the decision maker best responds to the strategies of the future selves. Hence a sophisticated equilibrium is essentially the subgame perfect equilibrium of the game played by the selves of the decision maker "against" each other.

An example given in Flesch, Kuipers, Mashiah-Yaakovi, Schoenmakers, Solan, and Vrieze (2010), discussed in detail in the following section, shows that in general a sophisticated equilibrium need not exist. This motivates us to consider more permissive solution concepts: naive $\epsilon-$ equilibrium and sophisticated $\epsilon$-equilibrium. A naive $\epsilon$-equilibrium is a strategy profile with the property that every player's strategy can be supported with a belief that makes this strategy and belief combination $\epsilon$-optimal. In a sophisticated $\epsilon$-equilibrium each player is assumed to play an $\epsilon$-best response to the strategies of the subsequent players. 
Our results are as follows. We show that for each $\epsilon>0$ there exists both a naive $\epsilon$-equilibrium and a sophisticated $\epsilon$-equilibrium. These existence results rely on mixed strategies. If we assume that each player's payoff function is upper semicontinuous, then there exist both a naive 0 -equilibrium in pure strategies and a sophisticated 0-equilibrium in pure strategies.

Herings and Rohde (2006) and Luttmer and Mariotti (2006) consider how time-inconsistent decision makers interact in a market environment and give sufficient conditions for equilibrium existence. Nevertheless, Gabrieli and Ghosal (2013) point out that under standard assumptions equilibria may fail to exist in such environments. The heart of the problem is the satiation of the induced preferences of sophisticated decision makers, and the examples of non-existence are robust. On the contrary, the equilibrium existence issues taken up in this chapter are at the level of the individual decision maker rather than the interaction between decision makers and existence problems can be solved by notions of $\epsilon$-equilibrium.

One of the key results in O'Donoghue and Rabin (1999) is that a sophisticated decision maker executes a task earlier than a naive decision maker. We provide a counterpart of this result in our setup. We show that for a given sophisticated $\epsilon$-equilibrium there exists a naive $\epsilon$-equilibrium with the probability of stopping not higher than in the given sophisticated $\epsilon$-equilibrium. Conversely, given a naive $\epsilon$-equilibrium there is a sophisticated $\epsilon$-equilibrium with the probability of stopping not smaller than in the given naive $\epsilon$-equilibrium.

Apart from the literature on time-inconsistent decision making, our results contribute to the literature on the existence of subgame perfect $\epsilon$-equilibrium in perfect information games, see e.g. Flesch et al. (2010), Purves and Sudderth (2011), and De Pril, Flesch, Kuipers, Schoenmakers, and Vrieze (2014). For the most part, this literature focuses on games 
with finitely many players. In contrast, here we consider a class of games played by infinitely many players.

Related to the infinite centipede games as considered here are socalled stopping games, see Solan (2005) and Mashiah-Yaakovi (2009). These are dynamic games where at each period of time each player can choose to stop or to continue. Our work is also related to intergenerational games, where there is a sequence of players such that each player represents an entire generation, see Phelps and Polak (1968) and Balbus, Jaśkiewicz, and Nowak (2015).

The chapter is organized as follows. In Section 2, we discuss the so-called procrastination game. The game serves to illustrate some of the non-trivial aspects of our analysis and to motivate the need for the solution concepts of naive $\epsilon$-equilibrium and sophisticated $\epsilon$-equilibrium. In Section 3, we introduce the general model and define naive and sophisticated $\epsilon$-equilibria. In Section 4 , we focus on a special class of games in which the payoffs are upper semi-continuous, and show the existence of naive 0-equilibrium and sophisticated 0-equilibrium in pure strategies. In Section 5, we examine the existence of naive $\epsilon$-equilibrium and, in Section 6, the existence of sophisticated $\epsilon$-equilibrium. In Section 7, we compare the stopping probabilities of naive and sophisticated decision makers and show that sophisticated decision makers stop earlier.

\subsection{The Procrastination Game}

Consider a decision maker who contemplates quitting smoking. On any given day the decision maker prefers quitting tomorrow to quitting today, and prefers quitting today to never quitting. This is an example of a decision maker with time-inconsistent preferences: quitting on day 2 is the best option from the perspective of day 1 , but it is no longer the best option once it is considered on day 2 itself. 
Following the standard approach to modeling time-inconsistent preferences, we represent the decision maker by a sequence of different selves, where day $t$ self makes a decision on behalf of the decision maker on day $t$. This leads us to the following game tree, where $S$ (stop) stands for quitting smoking and $C$ (continue) represents the option to postpone quitting:

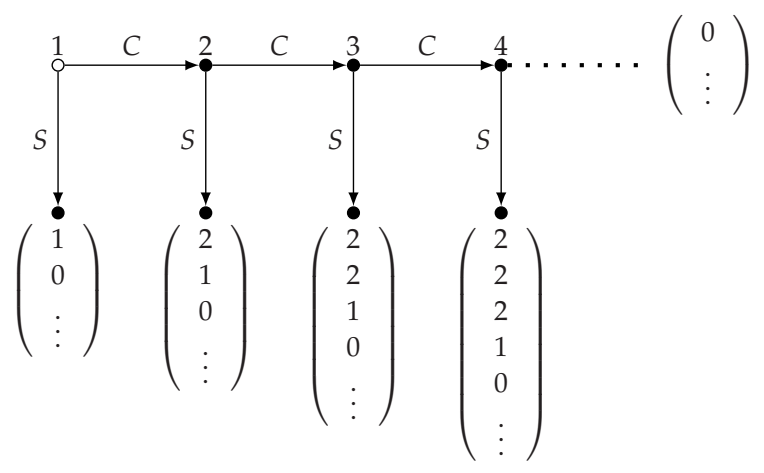

Figure 2.1: Procrastination game.

For the sake of concreteness we choose the following numerical values for the payoffs: the day $t$ self of the decision maker obtains a payoff of 1 if the decision maker quits on day $t$, a payoff of 2 if the the decision maker quits on any day $k>t$, and 0 in all other situations, so in particular if the decision maker never quits. We refer to this situation as the Procrastination game. O'Donoghue and Rabin (1999) consider a decision maker who has to decide when to execute a task such as quitting smoking. One important feature of their model is an exogenous deadline: once the deadline is reached, the decision maker has no choice but to execute the task. In contrast, the decision maker acting in the procrastination game above has the option to never quit. Our general model as detailed in the following section captures both cases with and without an exogenous deadline. 
Whether the decision maker quits, and if so, when, depends on his type. Following much of the literature on time-inconsistent decision making we distinguish two types of decision makers: naive and sophisticated.

A naive decision maker acts under the erroneous assumption that his current self controls all future decisions in the game. Thus a naive decision maker intends to follow a strategy that maximizes his payoff over the entire continuation game, but in reality only carries out the first action. In the procrastination game, this behavior implies never quitting as the decision maker always intends to quit later.

A sophisticated decision maker, in contrast, is fully aware that his day $t$ self only controls the decision on day $t$, and that future selves have different preferences. In the literature, this behavior is captured by the concept of sophisticated equilibrium, which essentially is nothing but a subgame perfect equilibrium of the game with multiple selves.

Somewhat surprisingly, the procrastination game has no sophisticated equilibrium in pure strategies, a fact already noticed (without proof) in Flesch et al. (2010), who introduced this game to show that properties of games with infinitely many players can be substantially different from those having finitely many players. For the sake of completeness, we give a short argument.

Claim 2.2.1. The Procastination game has no sophisticated equilibrium in pure strategies.

Proof. Suppose by way of contradiction that there is a sophisticated equilibrium in pure strategies. We distinguish three cases and derive a contradiction in each case. Let $I$ be the set of players who choose action $S$ conditional on reaching their decision nodes.

Case 1: Each player plays action $C$, so each player receives a payoff of 0 . Player 1 would get a payoff of 1 by deviating to $S$. 
Case 2: Exactly one player, say player $t$, chooses $S$. Since players $t+1, t+2, \ldots$ do not belong to $I$, player $t+1$ receives a payoff of 0 in the subgame starting in time $t+1$. A deviation to $S$ gives player $t+1$ a payoff of 1 .

Case 3: There exist two distinct players, say $t_{1}$ and $t_{2}$, who are elements of $I$. Without loss of generality, suppose $t_{1}<t_{2}$. In the subgame starting in time $t_{1}$, player $t_{1}$ receives a payoff of 1 but he would get 2 by deviating to $C$.

We show in Claim 6.1 that the procrastination game has no sophisticated equilibrium even when mixed strategies are considered. Nonexistence of a sophisticated equilibrium in the procrastination game motivates us to consider approximate solution concepts: naive $\epsilon$-equilibrium and sophisticated $\epsilon$-equilibrium. Under both concepts, the decision maker is assumed to maximize his payoff up to a margin of $\epsilon$. As we demonstrate in Section 6, the procrastination game does have a sophisticated $\epsilon$-equilibrium for each positive $\epsilon$, namely the strategy profile whereby each self stops with probability $\epsilon$.

\subsection{The General Model}

In this section, we describe infinite centipede games and define two solution concepts: naive $\epsilon$-equilibrium and sophisticated $\epsilon$-equilibrium.

In an infinite centipede game $G$, the set of players is the set $\mathbb{N}$ of natural numbers and the set of actions is $A=\{C, S\}$, where $C$ stands for continue and $S$ stands for stop. The game is played as follows. At time 1, player 1 chooses an action. If he chooses action $S$, then the game ends. If he chooses action $C$, then the play proceeds to time 2 where player 2 chooses an action. This is repeated as long as players choose action $C$. 
The payoff for player $i \in \mathbb{N}$ is $a_{t}^{i} \in \mathbb{R}$ if the game ends at time $t$ and $a_{\infty}^{i}$ if no one plays action $S$. We assume that payoffs are uniformly bounded, i.e.,

$$
B=\sup _{i \in \mathbb{N}} \sup _{t \in \mathbb{N}^{*}}\left|a_{t}^{i}\right|<\infty,
$$

where $\mathbb{N}^{*}$ stands for $\mathbb{N} \cup\{\infty\}$. By using the vector notation $a_{t}=\left(a_{t}^{i}\right)_{i \in \mathbb{N}}$ for every $t \in \mathbb{N}^{*}$, a centipede game can be represented as in Figure 2.2.

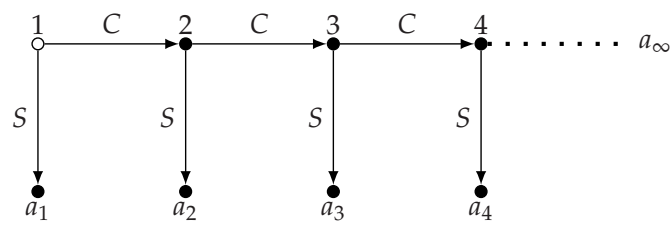

Figure 2.2: An infinite centipede game.

Our model can easily encompass various forms of discounted utility. For instance, when for all players the choice of $C$ during the first $t$ days yields instantaneous benefits $b_{t} \geq 0$ in day $t$, the instantaneous costs of stopping in day $t$ are $c_{t} \geq 0$, after stopping no further instantaneous costs and benefits occur, and player $i$ discounts benefits and costs in day $t$ by the discount factor $\delta_{t}^{i} \in[0,1]$, then we have

$$
a_{t}^{i}=\sum_{k=1}^{t-1} \delta_{k}^{i} b_{k}-\delta_{t}^{i} c_{t} .
$$

If discounting takes the standard exponential form, $\delta_{t}^{i}=(\delta)^{t}$ for some $\delta \in(0,1)$, then boundedness of the sequences $b_{t}$ and $c_{t}$ is sufficient for $a_{\infty}^{i}=\sum_{k=1}^{\infty} \delta_{k}^{i} b_{k}$ to be well-defined and to obtain uniform boundedness as expressed in (2.1).

A strategy for player $i$ is a probability distribution $\sigma^{i}$ on the set of actions $\{C, S\}$. The interpretation is that, if time $i$ is reached, then $\sigma^{i}$ recommends to play $C$ with probability $\sigma^{i}(C)$ and to play $S$ with probability $\sigma^{i}(S)$. The set of strategies for player $i$ is denoted by $\Sigma^{i}$ and the set 
of strategy profiles is denoted by $\Sigma=\mathrm{X}_{i \in \mathbb{N}} \Sigma^{i}$. A strategy $\sigma^{i}$ of player $i$ is uniquely specified by the probability to stop, $\sigma^{i}(S)$. Hence $\Sigma^{i}$ can be identified with $[0,1]$, and $\Sigma$ can be identified with $[0,1]^{\mathbb{N}}$.

A strategy $\sigma^{i}$ is called pure if either $\sigma^{i}(S)=0$ or $\sigma^{i}(S)=1$. Hence a pure strategy is an element of $\{0,1\}$ and a pure strategy profile is an element of $\{0,1\}\}^{\mathbb{N}}$.

Let $\sigma$ be a strategy profile. The expected utility of player $i$, conditional on the game not being stopped before time $t$, is denoted by $u^{i}(\sigma \mid t)$ and can be calculated as:

$$
u^{i}(\sigma \mid t)=\sigma^{t}(S) \cdot a_{t}^{i}+\sum_{k=t+1}^{\infty} \sigma^{k}(S) \prod_{j=t}^{k-1} \sigma^{j}(C) \cdot a_{k}^{i}+\prod_{j=t}^{\infty} \sigma^{j}(C) \cdot a_{\infty}^{i} .
$$

Note that $u^{i}(\sigma \mid i+1)$ is the expected utility of player $i$ when player $i$ plays action $C$ at time $i$.

We now define the concept of naive $\epsilon$-equilibrium.

Definition 2.3.1. Let $\epsilon \geq 0$. A strategy profile $\tau^{*} \in \Sigma$ is called a naive $\epsilon$ equilibrium if there exists a sequence $\left(\tau_{i}\right)_{i \in \mathbb{N}}$ of strategy profiles satisfying the following two conditions:

1. $\tau^{*, i}=\tau_{i}^{i}$ for every player $i \in \mathbb{N}$,

2. $u^{i}\left(\tau_{i} \mid i\right) \geq u^{i}(\sigma \mid i)-\epsilon$ for every player $i \in \mathbb{N}$ and every strategy profile $\sigma \in \Sigma$.

A naive 0-equilibrium is simply called a naive equilibrium.

The idea behind Definition 2.3.1 originates with the literature on time-inconsistent decision making. Thus suppose that, as in the procrastination game of the previous section, player $i$ represents the day $i$ self of a decision maker. The strategy profile $\tau_{i}$ can then be thought of as the complete course of actions that the day $i$ self intends to carry out. Condition 2 says that $\tau_{i}$ is an $\epsilon$-optimal strategy profile in the continuation game when evaluated against day $i$ 's preferences. 
The decision maker is naive as he fails to realize that his day $i$ self only controls the decision on day $i$, and that the strategy profile $\tau_{i}$ need not be $\epsilon$-optimal for the future selves. As a result, the sequence of strategies that the naive decision maker actually carries out is $\left(\tau_{1}^{1}, \tau_{2}^{2}, \ldots\right)$. This sequence is exactly $\tau^{*}$ by Condition 1 of Definition 2.3.1. Thus $\tau^{*}$ could be thought of as the realized behavior of a naive decision maker.

Naive $\epsilon$-equilibrium could also be interpreted without a recourse to time-inconsistent decision making. It represents a situation in which player $i$ fails to take into account the fact that he only controls a single decision node at time $i$, subsequent decisions being taken by other players.

For each player $i \in \mathbb{N}$, we define $A^{i}=\left\{a_{i}^{i}, a_{i+1}^{i}, \ldots\right\} \cup\left\{a_{\infty}^{i}\right\}$, which is the set of possible payoffs for player $i$ conditional on the fact that no player has stopped the game before him. Also, we let

$$
M^{i}=\sup A^{i} .
$$

With this notation, Condition 2 of Definition 2.3.1 is equivalent to

$$
u^{i}\left(\tau_{i} \mid i\right) \geq M^{i}-\epsilon \text { for every player } i \in \mathbb{N} .
$$

As an illustration, consider the Procrastination game in Figure 2.1. In this game, $M^{i}=2$ for each player $i$. It can be verified that the naive $\epsilon$ equilibria are exactly those strategy profiles $\tau^{*}$ for which $\tau^{*, i}(C) \geq 1-\epsilon$ for each player $i$.

Definition 2.3.2. Let $\epsilon \geq 0$. A strategy profile $\sigma^{*} \in \Sigma$ is called a sophisticated $\epsilon$-equilibrium if for each player $i \in \mathbb{N}$ and each strategy $\sigma^{i} \in \Sigma^{i}$ :

$$
u^{i}\left(\sigma^{*} \mid i\right) \geq u^{i}\left(\left(\sigma^{i}, \sigma^{*,-i}\right) \mid i\right)-\epsilon .
$$

A sophisticated 0-equilibrium is simply called a sophisticated equilibrium.

A sophisticated decision maker is fully aware that his day $i$ self only controls the decision on day $i$ and that the future selves may have 
different preferences. Thus in a sophisticated $\epsilon$-equilibrium each self of the decision maker $\epsilon$-best responds to the strategies of the future selves. Hence a sophisticated $\epsilon$-equilibrium is essentially a subgame perfect $\epsilon$-equilibrium of the game.

If player $i^{\prime}$ s opponents play according to $\sigma^{*,-i}$ and player $i$ chooses action $C$ at time $i$, then his payoff is $u^{i}\left(\sigma^{*} \mid i+1\right)$, whereas if player $i$ chooses action $S$, then his payoff is $a_{i}^{i}$. Hence, a strategy profile $\sigma^{*}$ is a sophisticated $\epsilon$-equilibrium if and only if $\sigma^{*}$ satisfies the following two inequalities for every player $i$ :

$$
\begin{gathered}
u^{i}\left(\sigma^{*} \mid i\right) \geq u^{i}\left(\sigma^{*} \mid i+1\right)-\epsilon, \\
u^{i}\left(\sigma^{*} \mid i\right) \geq a_{i}^{i}-\epsilon .
\end{gathered}
$$

In our illustrative example, the Procrastination game in Figure 2.1, there is no sophisticated $\epsilon$-equilibrium in pure strategies for $\epsilon \in[0,1)$ and no sophisticated equilibrium as we will show in Section 6. On the other hand, for $\epsilon>0$, we will provide a proof that this game does admit a sophisticated $\epsilon$-equilibrium in mixed strategies, where each player stops with probability $\epsilon$.

\subsection{Existence of Equilibrium in the Upper Semicon- tinuous Case}

In this section, we establish the existence of naive and sophisticated equilibria if the payoffs in the game are upper semi-continuous, i.e., if for every player $i \in \mathbb{N}$

$$
\limsup _{t \rightarrow \infty} a_{t}^{i} \leq a_{\infty}^{i} .
$$

The payoffs of player $i$ are upper semi-continuous if the payoffs of player $i$ when stopping the game at time $t$ with $t$ going to infinity are less than or equal to the payoffs obtained when never stopping the game. 
A typical example of payoffs that are upper semi-continuous but not continuous concerns the case where stopping the game corresponds to making a costly investment, say for instance in the reduction of carbon dioxide emissions. Making the investment too late leaves too little time to recoup the costs or would not change a disastrous outcome. In such a case, not making the investment at all would be preferred to making the investment at a very late point in time. Another example of payoffs that are upper semi-continuous but not continuous results when stopping corresponds to giving in and the player derives a positive psychological benefit from never doing so. Continuity, and therefore upper semicontinuity, is satisfied in the standard model of exponential discounting when instantaneous costs and benefits are uniformly bounded.

First, we provide necessary and sufficient conditions for the existence of naive equilibrium without continuity assumptions on the payoffs.

Theorem 2.4.1. The following statements are equivalent:

(i) For every player $i \in \mathbb{N}$, the set $A^{i}=\left\{a_{i}^{i}, a_{i+1}^{i}, \ldots\right\} \cup\left\{a_{\infty}^{i}\right\}$ has a maximum.

(ii) There exists a naive equilibrium in pure strategies.

(iii) There exists a naive equilibrium.

Proof. $\left(i \rightarrow\right.$ ii) Consider a player $i$. If the maximum of $A^{i}$ is $a_{\infty}^{i}$, then let $\tau_{i}$ be the pure strategy profile that always chooses action $C$. If the maximum of $A^{i}$ is not $a_{\infty}^{i}$, but some $a_{t}^{i}$ with $t \in \mathbb{N}$, then let $\tau_{i}$ be the pure strategy profile that always chooses action $C$, except at time $t$, where it chooses action $S$. It is clear that the pure strategy profile $\tau^{*}$ defined by $\tau^{*, i}=\tau_{i}^{i}$ for every $i \in \mathbb{N}$ is a naive equilibrium.

$($ ii $\rightarrow$ iii) Obvious.

$($ iii $\rightarrow i$ ) Suppose that there exists a naive equilibrium. Take a player $i$. By (2.3), there exists a strategy profile $\tau_{i}$ such that $u^{i}\left(\tau_{i} \mid i\right) \geq M^{i}$. It follows from (2.2) that $u^{i}\left(\tau_{i} \mid i\right)=M^{i}$ and that there exists a $t \in\{i, i+1, \ldots\} \cup\{\infty\}$ such that $a_{t}^{i}=M^{i}$. Thus, the set $A^{i}$ has a maximum. 
In view of the above theorem, a naive equilibrium does not always exist. A concrete example is the game in Figure 2.3, which we will consider later. However, we have the following existence result for games with upper semicontinuous payoffs.

Theorem 2.4.2. There exists a naive equilibrium in pure strategies if the payoffs are upper semicontinuous.

Proof. Due to (2.6), the set $A^{i}=\left\{a_{i}^{i}, a_{i+1}^{i}, \ldots\right\} \cup\left\{a_{\infty}^{i}\right\}$ has a maximum for every player $i \in \mathbb{N}$. Therefore, by Theorem 2.4.1, there exists a naive equilibrium in pure strategies.

Now we turn to the existence of a sophisticated equilibrium. The proof of the following result employs a truncation approach similar to that in Fudenberg and Levine (1983). One crucial difference however is that we do not assume the payoffs to be continuous, but only upper semicontinuous.

Theorem 2.4.3. There exists a sophisticated equilibrium in pure strategies if the payoffs are upper semicontinuous.

Proof. Consider a centipede game $G$. For every $T \in \mathbb{N}$, we define the $T$-period truncated game $G_{T}$ which is identical to $G$ except for one modification: if all players $1, \ldots, T$ choose to continue, then, regardless of future play, the payoff of each player $i \in \mathbb{N}$ is equal to $a_{T+1}^{i}$. Since the payoffs cannot change after time $T$, this game is essentially a $T$-period game.

For every $T \in \mathbb{N}$, the truncated game $G_{T}$ admits a pure sophisticated equilibrium $\sigma_{T}$ in which $\sigma_{T}^{i}(S)=1$ for every player $i \geq T+1$. Indeed, due to the payoffs in $G_{T}$, we can set $\sigma_{T}^{i}(S)=1$ for every player $i \geq T+1$ and then determine $\sigma_{T}^{T}(S), \ldots, \sigma_{T}^{1}(S)$ by backward induction. If a player is indifferent between playing action $C$ and action $S$ then either action can be taken. The set of pure strategy profiles, as mentioned earlier, can 
be identified with the infinite Cartesian product $\{0,1\}^{\mathbb{N}}$ and is thus a compact metrizable topological space. Hence the sequence $\left(\sigma_{T}\right)_{T=1}^{\infty}$ has an accumulation point $\bar{\sigma} \in\{0,1\}^{\mathbb{N}}$. By taking a subsequence if necessary, we can assume that $\left(\sigma_{T}\right)_{T=1}^{\infty}$ converges to the strategy profile $\bar{\sigma}$. We distinguish two cases.

Case 1: Suppose that there are only finitely many players who play action $S$ in the strategy profile $\bar{\sigma}$. So, there exists a time $t$ such that for all players $i \geq t, \bar{\sigma}^{i}(C)=1$. We prove that $\bar{\sigma}$ induces a sophisticated equilibrium for the subgame of $G$ starting at time $t$. So we need to show that $u^{i}(\bar{\sigma} \mid i) \geq a_{i}^{i}$ for all $i \geq t$. For every $i, T \in \mathbb{N}$, let

$$
m_{T}^{i}=\min \left\{k \geq i \mid \sigma_{T}^{k}(S)=1\right\},
$$

so $m_{T}^{i}$ is the first player at time $i$ or later who stops in the strategy profile $\sigma_{T}$. We have for every $i \geq t$ that

$$
\begin{aligned}
u^{i}(\bar{\sigma} \mid i) & =a_{\infty}^{i} \\
& \geq \limsup _{T \rightarrow \infty} a_{T}^{i} \\
& \geq \limsup _{T \rightarrow \infty} a_{m_{T}^{i}}^{i} \\
& =\limsup _{T \rightarrow \infty} u^{i}\left(\sigma_{T} \mid i\right) \\
& \geq a_{i}^{i} .
\end{aligned}
$$

Equality (2.7) holds, since no one stops from time $t$ onwards in the strategy profile $\bar{\sigma} ;$ (2.8) follows from the assumption of the theorem; (2.9) follows from the definition of limit superior, because $m_{T}^{i} \rightarrow \infty$ as $T \rightarrow \infty$, for every $i \geq t$; (2.10) holds as $u^{i}\left(\sigma_{T} \mid i\right)=a_{m_{T}^{i}}^{i}$ for every $i$ and $T$; and finally (2.11) is true since $\sigma_{T}$ is a sophisticated equilibrium in $G_{T}$. Hence, we have $u^{i}(\bar{\sigma} \mid i) \geq a_{i}^{i}$ for all $i \geq t$, as desired. This means that $\bar{\sigma}$ induces a sophisticated equilibrium for the subgame of $G$ starting at time $t$. Now we can use backward induction from time $t$ to obtain a sophisticated equilibrium in $G$. 
Case 2 : Suppose that there are infinitely many players who play action $S$ in the strategy profile $\bar{\sigma}$. Take an arbitrary player $i$. Let

$$
n^{i}=\min \left\{k>i \mid \bar{\sigma}^{k}(S)=1\right\},
$$

so $n^{i}$ is the first player at time $i+1$ or later who stops in the strategy profile $\bar{\sigma}$. Since $\left(\sigma_{T}\right)_{T=1}^{\infty}$ converges to $\bar{\sigma}$, there exists $T \geq n^{i}$ such that for all $j \leq n^{i}$ we have $\sigma_{T}^{j}=\bar{\sigma}^{j}$. Because $\sigma_{T}$ is a sophisticated equilibrium in the game $G_{T}$, player $i$ does not have a profitable deviation from $\sigma_{T}$ in $G_{T}$. It follows that player $i$ does not have a profitable deviation from $\bar{\sigma}$ in the game $G$. We conclude that $\bar{\sigma}$ is a sophisticated equilibrium of $G$.

\subsection{Existence of Naive $\epsilon$-Equilibrium}

We know from the previous section that a naive equilibrium does not always exist. The following theorem deals with the existence of naive $\epsilon$-equilibrium.

Theorem 2.5.1. For every $\epsilon>0$, there exists a naive $\epsilon$-equilibrium in pure strategies.

Proof. Let $\epsilon>0$ be given. Consider a player $i$. We distinguish two cases in order to define a pure strategy profile $\tau_{i}$.

Case 1: $a_{\infty}^{i} \geq M^{i}-\epsilon$, where $M^{i}$ is given in (2.2). In this case, let $\tau_{i}$ be the pure strategy profile that always chooses action $C$.

Case 2: $a_{\infty}^{i}<M^{i}-\epsilon$. In this case, there exists $t \in\{i, i+1, \ldots\}$ such that $a_{t}^{i} \geq M^{i}-\epsilon$. Let $\tau_{i}$ be the pure strategy profile that always chooses action $C$, except at time $t$, where it chooses action $S$.

Now define the pure strategy profile $\tau^{*}$ by setting $\tau^{*, i}=\tau_{i}^{i}$ for every player $i \in \mathbb{N}$. Then, the strategy profiles $\tau^{*}$ and $\tau_{i}$, for every $i \in \mathbb{N}$, satisfy Condition 1 of Definition 2.3.1 and inequality (2.3), so $\tau^{*}$ is a naive $\epsilon$-equilibrium. 
As an illustration, consider a game where the payoff for player $i \in \mathbb{N}$ is $1-1 /(t-i+1)$ if the game ends at time $t>i$, and 0 if the game ends at time $t \leq i$ or if no one stops. The game is given in Figure 2.3.

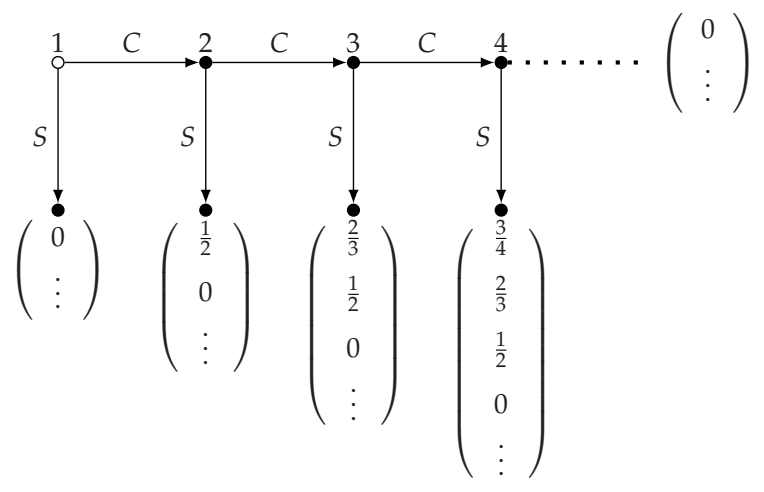

Figure 2.3: A game without naive equilibrium.

By Theorem 2.4.1, there is no naive equilibrium in this game. On the other hand, the pure strategy profile $\tau^{*}$ that always chooses action $C$ is a naive $\epsilon$-equilibrium for every $\epsilon>0$. Indeed, let $\epsilon>0$. For every player $i$, take a time $t_{i}$ such that $t_{i}>i$ and $a_{t_{i}}^{i} \geq 1-\epsilon$. Define $\tau_{i}$ to be the pure strategy profile that always chooses action $C$, except at time $t_{i}$, where it chooses action $S$. Then, for every player $i \in \mathbb{N}$ it holds that $\tau^{*, i}=\tau_{i}^{i}$ and inequality (2.3) is satisfied, so $\tau^{*}$ is a naive $\epsilon$-equilibrium as claimed. Notice also that the strategy profile $\tau^{*}$ is a sophisticated equilibrium of the game.

\subsection{Existence of Sophisticated $\epsilon$-Equilibrium}

In this section, we examine the existence of a sophisticated $\epsilon$-equilibrium. The following claim establishes that the Procrastination game has neither a sophisticated equilibrium nor a pure sophisticated $\epsilon$-equilibrium. 
Claim 2.6.1. The Procrastination game in Figure 2.1 has the following properties:

1. It admits no sophisticated $\epsilon$-equilibrium in pure strategies for any $\epsilon \in$ $[0,1)$.

2. It admits no sophisticated equilibrium.

Proof. First we prove part 1 . Take an $\epsilon \in[0,1)$ and suppose by way of contradiction that $\sigma$ is a sophisticated $\epsilon$-equilibrium in pure strategies. Consider some player $i \in \mathbb{N}$. For any pure strategy $\tau^{i}$ it holds that $u^{i}(\sigma \mid i) \geq u^{i}\left(\left(\tau^{i}, \sigma^{-i}\right) \mid i\right)-\epsilon$. Because pure strategy profiles can only induce payoffs 0,1 or 2 to any player in the game and because $\epsilon<1$, we must have $u^{i}(\sigma \mid i) \geq u^{i}\left(\left(\tau^{i}, \sigma^{-i}\right) \mid i\right)$. Therefore, pure strategy profile $\sigma$ is a sophisticated equilibrium. This contradicts Claim 2.2.1.

Now we prove part 2. Assume to the contrary that $\sigma$ is a sophisticated equilibrium. For every player $k$, let

$$
p(\sigma \mid k)=\prod_{i=k}^{\infty} \sigma^{i}(C)
$$

be the probability that the game never stops, provided that it has not been stopped before time $k$ and that the players play according to $\sigma$.

Assume first that there are two players $i$ and $j$, with $i<j$, such that $\sigma^{i}$ and $\sigma^{j}$ are not pure. Since $\sigma^{i}$ is not pure, we have $u^{i}\left(\left(C, \sigma^{-i}\right) \mid i\right)=$ $u^{i}\left(\left(S, \sigma^{-i}\right) \mid i\right)$. Note that $u^{i}\left(\left(S, \sigma^{-i}\right) \mid i\right)=1$ and

$$
u^{i}\left(\left(C, \sigma^{-i}\right) \mid i\right)=(1-p(\sigma \mid i+1)) \cdot 2,
$$

so $p(\sigma \mid i+1)=0.5$. By a similar argument, we obtain for player $j$ that $p(\sigma \mid j+1)=0.5$. But then

$$
p(\sigma \mid i+1)=\sigma^{i+1}(C) \sigma^{i+2}(C) \cdots \sigma^{j}(C) p(\sigma \mid j+1)
$$

yields $\sigma^{j}(C)=1$, which is a contradiction to the fact that $\sigma^{j}$ is not pure. 
Therefore, there is a time $t$ such that in the subgame that starts at $t$, the strategy profile $\sigma$ is a sophisticated equilibrium in pure strategies. By backward induction, we can then construct a sophisticated equilibrium in pure strategies for the whole game. This is however in contradiction with Claim 2.2.1.

The main result of this section is the following theorem on the existence of a sophisticated $\epsilon$-equilibrium.

Theorem 2.6.2. For every $\epsilon>0$, there exists a sophisticated $\epsilon$-equilibrium.

Proof. Take a centipede game G. Recall the definition of $B$ from (2.1). Choose a number $\delta$ so that $0<\delta \leq \min \left\{\frac{\epsilon}{2 B}, 1\right\}$. Define the normal-form game $G^{*}$ as the game with set of players $\mathbb{N}$, set of strategies for player $i \in \mathbb{N}$ equal to

$$
\Sigma^{* i}=\left\{\sigma^{i} \in \Sigma^{i} \mid \sigma^{i}(S) \in[\delta, 1]\right\},
$$

and utility function $v^{i}$ of player $i \in \mathbb{N}$ defined by

$$
v^{i}(\sigma)=u^{i}(\sigma \mid i)
$$

for each $\sigma$ in $\Sigma^{*}=\times_{i \in \mathbb{N}} \Sigma^{* i}$. The strategy sets of all players are nonempty, convex, and compact. Each player's utility function is affine, so quasi-concave, in that player's own strategy, since

$$
v^{i}(\sigma)=\sigma^{i}(S) a_{i}^{i}+\left(1-\sigma^{i}(S)\right) u^{i}(\sigma \mid i+1),
$$

where $u^{i}(\sigma \mid i+1)$ is unaffected by the strategy $\sigma^{i}$ of player $i$. Crucially, each player's utility function is continuous on $\Sigma^{*}$. The key to the continuity of the payoff functions is that under any strategy profile in $\Sigma^{*}$, the probability that no one ever stops is zero.

The game $G^{*}$ therefore has a Nash equilibrium, say $\sigma^{*}$, by Theorem 6.2 of Peleg and Yaari (1973). In view of equation (2.12), if $u^{i}\left(\sigma^{*} \mid i+\right.$ 1) $<a_{i}^{i}$ then $\sigma^{*, i}(S)=1$, and if $u^{i}\left(\sigma^{*} \mid i+1\right)>a_{i}^{i}$ then $\sigma^{*, i}(S)=\delta$. 
We show that $\sigma^{*}$ is a sophisticated $\epsilon$-equilibrium of $G$. Take any player $i \in \mathbb{N}$. If $u^{i}\left(\sigma^{*} \mid i+1\right)<a_{i}^{i}$ then $\sigma^{*, i}(S)=1$, so $u^{i}\left(\sigma^{*} \mid i\right)=v^{i}\left(\sigma^{*}\right)=a_{i}^{i}$ and (2.4) and (2.5) are satisfied. If $u^{i}\left(\sigma^{*} \mid i+1\right)=a_{i}^{i}$ then $u^{i}\left(\sigma^{*} \mid i\right)=v^{i}\left(\sigma^{*}\right)=$ $a_{i}^{i}$, so (2.4) and (2.5) are satisfied. So assume that $u^{i}\left(\sigma^{*} \mid i+1\right)>a_{i}^{i}$. We have that $\sigma^{*, i}(S)=\delta$, so

$$
\begin{aligned}
u^{i}\left(\sigma^{*} \mid i\right) & =v^{i}\left(\sigma^{*}\right) \\
& =\sigma^{*, i}(S) a_{i}^{i}+\left(1-\sigma^{*, i}(S)\right) u^{i}\left(\sigma^{*} \mid i+1\right) \\
& =\delta a_{i}^{i}+(1-\delta) u^{i}\left(\sigma^{*} \mid i+1\right) \\
& =\delta\left(a_{i}^{i}-u^{i}\left(\sigma^{*} \mid i+1\right)\right)+u^{i}\left(\sigma^{*} \mid i+1\right) \\
& \geq-\delta 2 B+u^{i}\left(\sigma^{*} \mid i+1\right) \\
& \geq u^{i}\left(\sigma^{*} \mid i+1\right)-\epsilon .
\end{aligned}
$$

Hence, inequality (2.4) is satisfied. Furthermore, it holds that

$$
\begin{aligned}
u^{i}\left(\sigma^{*} \mid i\right)-a_{i}^{i} & =v^{i}\left(\sigma^{*}\right)-a_{i}^{i} \\
& =\sigma^{*, i}(S) a_{i}^{i}+\left(1-\sigma^{*, i}(S)\right) u^{i}\left(\sigma^{*} \mid i+1\right)-a_{i}^{i} \\
& =\left(1-\sigma^{*, i}(S)\right)\left(u^{i}\left(\sigma^{*} \mid i+1\right)-a_{i}^{i}\right) \\
& \geq 0,
\end{aligned}
$$

where the inequality follows by $u^{i}\left(\sigma^{*} \mid i+1\right)>a_{i}^{i}$. Hence, inequality (2.5) is also satisfied.

We remark that one could give a direct proof of the theorem using truncations of the game tree. To do so, let $G_{T}$ be the game as in the proof of Theorem 3.21. One could show that $G_{T}$ has a sophisticated $\epsilon$-equilibrium $\sigma_{T}$ that is an element of $\Sigma^{*}$. One can further show that any accumulation point of the sequence $\left\{\sigma_{T}\right\}_{T \in \mathbb{N}}$ is a sophisticated $\epsilon-$ equilibrium of the original game $G$.

According to the definition of $B$ in (2.1), the payoffs are uniformly bounded. One might wonder whether it would be enough to assume only 
that the payoffs are bounded for each player separately. The following example shows that this weaker assumption would not suffice for the existence of a sophisticated $\epsilon$-equilibrium.

Consider the game with the following payoffs for every player $i$ : If the game ends before time $i$ then player $i$ 's payoff is 0 . If the game ends at time $i$ then player $i^{\prime}$ s payoff is $2^{i}$. If the game ends after time $i$ then player $i$ 's payoff is $2^{i+1}$. Finally, if the game never ends then player $i$ 's payoff is 0 . The game tree is given in Figure 2.4.

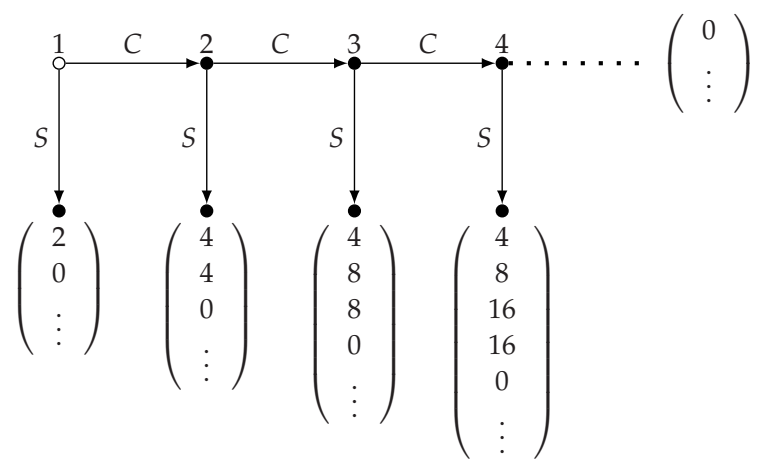

Figure 2.4: A centipede game without a sophisticated $\epsilon$-equilibrium.

It is clear that, for each $i \in \mathbb{N}$, sup $\left|a_{t}^{i}\right|=2^{i+1}$. At the same time it holds that sup sup $\left|a_{t}^{i}\right|=\infty$. $i \in \mathbb{N} t \in \mathbb{N}^{*}$

Claim 2.6.3. For each $\epsilon>0$, the game in Figure 2.4 admits no sophisticated e-equilibrium.

Proof. Take $\epsilon>0$ and suppose by way of contradiction that there is a sophisticated $\epsilon$-equilibrium $\sigma$ for the game in Figure 2.4. As in the proof of Claim 6.1, let $p(\sigma \mid k)=\prod_{i=k}^{\infty} \sigma^{i}(C)$ denote the probability that the game never stops, given that it has not stopped before time $k$ and the players follow the strategy profile $\sigma$. 
Take a player $i \in \mathbb{N}$. Since $\sigma$ is a sophisticated $\epsilon$-equilibrium, inequality (2.5) implies that $u^{i}(\sigma \mid i) \geq 2^{i}-\epsilon$. On the other hand, since $2^{i+1}$ is the highest payoff player $i$ can get and since he gets 0 if the game never stops, we have the following upper bound on the payoff: $u^{i}(\sigma \mid i) \leq(1-p(\sigma \mid i)) \cdot 2^{i+1}+p(\sigma \mid i) \cdot 0$. Combining these facts and rearranging terms, we find that

$$
p(\sigma \mid i) \leq \frac{1}{2}+\frac{\epsilon}{2^{i+1}} .
$$

Notice that the sequence $\{p(\sigma \mid i)\}_{i \in \mathbb{N}}$ is non-decreasing and bounded and hence has a limit. Furthermore, the preceding inequality implies that

$$
\lim _{i \rightarrow \infty} p(\sigma \mid i) \leq \frac{1}{2}
$$

Consider any player $i \in \mathbb{N}$. For each $j>i$ it holds that

$$
p(\sigma \mid i)=\prod_{t=i}^{\infty} \sigma^{t}(C)=\prod_{t=i}^{j-1} \sigma^{t}(C) \cdot p(\sigma \mid j)
$$

Taking the limit as $j$ approaches infinity, we obtain

$$
p(\sigma \mid i)=\lim _{j \rightarrow \infty} \prod_{t=i}^{j-1} \sigma^{t}(C) \cdot \lim _{j \rightarrow \infty} p(\sigma \mid j)=p(\sigma \mid i) \cdot \lim _{j \rightarrow \infty} p(\sigma \mid j) \leq p(\sigma \mid i) \cdot \frac{1}{2},
$$

which implies that $p(\sigma \mid i)=0$.

Thus for each $i \in \mathbb{N}$ it holds that $u^{i}(\sigma \mid i)=\sigma^{i}(S) \cdot 2^{i}+\left(1-\sigma^{i}(S)\right)$. $2^{i+1}$ and $u^{i}(\sigma \mid i+1)=2^{i+1}$. Since $\sigma$ is a sophisticated $\epsilon$-equilibrium, it holds by inequality (2.4) that $u^{i}(\sigma \mid i) \geq u^{i}(\sigma \mid i+1)-\epsilon$, and therefore $\sigma^{i}(S) \leq 2^{-i} \epsilon$.

Now take $t \in \mathbb{N}$ such that $2^{1-t} \epsilon<1$. Since $1-p(\sigma \mid t)$ is the probability that the game eventually stops conditional on time $t$ being reached, we have

$$
1-p(\sigma \mid t)=\sum_{j=t}^{\infty} \sigma^{j}(S) \prod_{i=t}^{j-1} \sigma^{i}(C) \leq \sum_{j=t}^{\infty} \sigma^{j}(S) \leq \sum_{j=t}^{\infty} 2^{-j} \epsilon \leq 2^{1-t} \epsilon,
$$

so $p(\sigma \mid t) \geq 1-2^{1-t} \epsilon>0$, contradicting $p(\sigma \mid t)=0$. 


\subsection{Sophisticates Stop Earlier}

One of the key results in O'Donoghue and Rabin (1999) is that a sophisticated decision maker executes a task earlier than a naive decision maker. In this section we derive the counterpart of this result in our setup. The comparison of sophisticated and naive decision makers in our setup is somewhat complicated by the fact that in general there might exist multiple sophisticated $\epsilon$-equilibria and multiple naive $\epsilon-$ equilibria. We thus have to compare two sets of equilibria. We achieve this by showing that [1] given a sophisticated $\epsilon$-equilibrium there exists a naive $\epsilon$-equilibrium with the probability to stop not greater than in the sophisticated $\epsilon$-equilibrium at any given time, and [2] given a naive $\epsilon$-equilibrium there exists a sophisticated $\epsilon$-equilibrium with the probability to stop not smaller than in the naive $\epsilon$-equilibrium at any given time.

We restrict attention to the case $\epsilon>0$ to guarantee the existence of naive and sophisticated $\epsilon$-equilibria. Indeed, recall that the Procrastination game of section 2 has no sophisticated equilibrium (see Claim 2.6.1), while it does admit a naive equilibrium, namely playing $C$ at every period. The game depicted in Figure 2.3 admits a sophisticated equilibrium but not a naive equilibrium.

Theorem 2.7.1. Let some $\epsilon>0$ be given. For each sophisticated $\epsilon$-equilibrium $\sigma^{*}$, there exists a naive $\epsilon$-equilibrium $\tau^{*}$ such that for all $i \in \mathbb{N}, \tau^{*, i}(S) \leq$ $\sigma^{*, i}(S)$.

Proof. Let $\sigma^{*}$ be a sophisticated $\epsilon$-equilibrium. For every $i \in \mathbb{N}$, we define the strategy profile $\tau_{i} \in \Sigma$ as follows. If $u^{i}\left(\sigma^{*} \mid i\right) \geq M^{i}-\epsilon$, then let $\tau_{i}=\sigma^{*}$. Otherwise, it holds that $u^{i}\left(\sigma^{*} \mid i\right)<M^{i}-\epsilon$. As $a_{i}^{i}-\epsilon \leq u^{i}\left(\sigma^{*} \mid i\right)$ by inequality (2.5), it then holds that $a_{i}^{i}<M^{i}$. Consequently, there exists $t \in\{i+1, i+2, \ldots\} \cup\{\infty\}$ such that $a_{t}^{i} \geq M^{i}-\epsilon$. If $t=\infty$ then we define $\tau_{i}$ by letting $\tau_{i}^{j}(S)=0$ for all $j \in \mathbb{N}$, while if $t \in\{i+1, i+2, \ldots\}$ 
we define $\tau_{i}^{t}(S)=1$ and $\tau_{i}^{j}(S)=0$ for all $j \neq t$. It is easy to see that the strategy profile $\tau_{i}$ satisfies inequality (2.3). Now define $\tau^{*} \in \Sigma$ by letting $\tau^{*, i}=\tau_{i}^{i}$ for each $i \in \mathbb{N}$. Then $\tau^{*}$ is a naive $\epsilon$-equilibrium. Since $\tau^{*, i}(S)$ is either equal to $\sigma^{*, i}(S)$ or 0 , it holds for every $i \in \mathbb{N}$ that $\tau^{*, i}(S) \leq \sigma^{*, i}(S)$.

Theorem 2.7.2. Let some $\epsilon>0$ be given. For each naive $\epsilon$-equilibrium $\tau^{*}$, there exists a sophisticated $\epsilon$-equilibrium $\sigma^{*}$ such that for all $i \in \mathbb{N}, \tau^{*, i}(S) \leq$ $\sigma^{*, i}(S)$.

Proof. Fix a naive $\epsilon$-equilibrium $\tau^{*}$ and let $\left(\tau_{i}\right)_{i \in \mathbb{N}}$ be as in Definition 2.3.1. Choose a number $\delta$ so that $0<\delta \leq \min \left\{\frac{\epsilon}{2 B}, 1\right\}$. For $i \in \mathbb{N}$ let $\pi^{i}=\max \left\{\delta, \tau^{*, i}(S)\right\}$. Define the normal-form game $G^{* *}$ as the game with set of players $\mathbb{N}$, set of strategies for player $i \in \mathbb{N}$ equal to

$$
\Sigma^{* * i}=\left\{\sigma^{i} \in \Sigma^{i} \mid \sigma^{i}(S) \in\left[\pi^{i}, 1\right]\right\},
$$

and utility function $v^{i}$ of player $i \in \mathbb{N}$ defined by

$$
v^{i}(\sigma)=u^{i}(\sigma \mid i)
$$

for each $\sigma$ in $\Sigma^{* *}=\times_{i \in \mathbb{N}} \Sigma^{* * i}$. The strategy sets of all players are nonempty, convex, and compact. The utility functions of all players are continuous on $\Sigma^{* *}$ and affine, so quasi-concave, in their own strategy. The game $G^{* *}$ therefore has a Nash equilibrium, say $\sigma^{*}$, by Theorem 6.2 of Peleg and Yaari (1973). The payoff functions can be written as

$$
v^{i}(\sigma)=\sigma^{i}(S) a_{i}^{i}+\left(1-\sigma^{i}(S)\right) u^{i}(\sigma \mid i+1),
$$

where $u^{i}(\sigma \mid i+1)$ is unaffected by the strategy $\sigma^{i}$ of player $i$. It follows that if $u^{i}\left(\sigma^{*} \mid i+1\right)<a_{i}^{i}$ then $\sigma^{*, i}(S)=1$, and if $u^{i}\left(\sigma^{*} \mid i+1\right)>a_{i}^{i}$ then $\sigma^{*, i}(S)=\pi^{i}$

We show that $\sigma^{*}$ is a sophisticated $\epsilon$-equilibrium of $G$.

Take any player $i \in \mathbb{N}$. If $u^{i}\left(\sigma^{*} \mid i+1\right)<a_{i}^{i}$ then $\sigma^{*, i}(S)=1$, so $u^{i}\left(\sigma^{*} \mid i\right)=v^{i}\left(\sigma^{*}\right)=a_{i}^{i}$ and (2.4) and (2.5) are satisfied. If $u^{i}\left(\sigma^{*} \mid i+1\right)=a_{i}^{i}$ 
then $u^{i}\left(\sigma^{*} \mid i\right)=v^{i}\left(\sigma^{*}\right)=a_{i}^{i}$, so (2.4) and (2.5) are satisfied. So assume that $u^{i}\left(\sigma^{*} \mid i+1\right)>a_{i}^{i}$. We have that $\sigma^{*, i}(S)=\pi^{i}$.

If $\pi^{i}=\delta$, then

$$
\begin{aligned}
u^{i}\left(\sigma^{*} \mid i\right) & =v^{i}\left(\sigma^{*}\right) \\
& =\sigma^{*, i}(S) a_{i}^{i}+\left(1-\sigma^{*, i}(S)\right) u^{i}\left(\sigma^{*} \mid i+1\right) \\
& =\delta a_{i}^{i}+(1-\delta) u^{i}\left(\sigma^{*} \mid i+1\right) \\
& \geq-\delta 2 B+u^{i}\left(\sigma^{*} \mid i+1\right) \\
& \geq u^{i}\left(\sigma^{*} \mid i+1\right)-\epsilon .
\end{aligned}
$$

If $\pi^{i}=\tau^{*, i}(S)$, we have the following chain of inequalities:

$$
\begin{aligned}
u^{i}\left(\sigma^{*} \mid i\right)-u^{i}\left(\sigma^{*} \mid i+1\right) & =\sigma^{*, i}(S) a_{i}^{i}+\left(1-\sigma^{*, i}(S)\right) u^{i}\left(\sigma^{*} \mid i+1\right)-u^{i}\left(\sigma^{*} \mid i+1\right) \\
& =\sigma^{*, i}(S)\left(a_{i}^{i}-u^{i}\left(\sigma^{*} \mid i+1\right)\right) \\
& =\tau^{*, i}(S)\left(a_{i}^{i}-u^{i}\left(\sigma^{*} \mid i+1\right)\right) \\
& \geq \tau^{*, i}(S)\left(a_{i}^{i}-M^{i}\right) \\
& =\tau^{*, i}(S) a_{i}^{i}+\left(1-\tau^{*, i}(S)\right) M^{i}-M^{i} \\
& \geq \tau^{*, i}(S) a_{i}^{i}+\left(1-\tau^{*, i}(S)\right) u^{i}\left(\tau_{i} \mid i+1\right)-M^{i} \\
& =u^{i}\left(\tau_{i} \mid i\right)-M^{i} \\
& \geq-\epsilon
\end{aligned}
$$

where equality (2.13) holds since we assume $\sigma^{*, i}(S)=\pi^{i}=\tau^{*, i}(S)$. Inequality (2.14) holds since $u^{i}\left(\sigma^{*} \mid i+1\right)$ is an expectation of the payoffs under a probability distribution over the set $\left\{a_{i+1}^{i}, a_{i+2}^{i}, \cdots\right\} \cup\left\{a_{\infty}^{i}\right\}$, a subset of $A^{i}$, and hence is bounded above by $M^{i}=\sup A^{i}$. Inequality (2.15) follows since $u^{i}\left(\tau_{i} \mid i+1\right)$ is likewise bounded above by $M^{i}$, and inequality (2.16) follows from inequality (2.3). Hence, $\sigma^{*}$ satisfies inequality (2.4). 
Moreover, we have

$$
\begin{aligned}
u^{i}\left(\sigma^{*} \mid i\right)-a_{i}^{i} & =\sigma^{*, i}(S) a_{i}^{i}+\left(1-\sigma^{*, i}(S)\right) u^{i}\left(\sigma^{*} \mid i+1\right)-a_{i}^{i} \\
& =\left(1-\sigma^{*, i}(S)\right)\left(u^{i}\left(\sigma^{*} \mid i+1\right)-a_{i}^{i}\right) \\
& \geq 0,
\end{aligned}
$$

so inequality (2.5) is also satisfied.

Notice that the Theorems 2.7.1 and 2.7.2 do not preclude the possibility that there exist a naive $\epsilon$-equilibrium $\tau^{*}$ and a sophisticated $\epsilon$-equilibrium $\sigma^{*}$ such that $\tau^{*, i}(S)>\sigma^{*, i}(S)$ for all $i \in \mathbb{N}$. The trivial game where all payoffs are 0 would yield an example.

\subsection{Conclusion}

In this chapter, we have examined infinite centipede games with uniformly bounded payoffs. We have looked at two solution concepts, naive and sophisticated $\epsilon$-equilibria, depending on the type of the decision maker.

Regarding a naive decision maker, we show that there does not always exist a naive 0 -equilibrium. We provide the necessary and sufficient conditions for the existence of a naive 0 -equilibrium and show that upper semicontinuity of payoffs is sufficient for existence. Finally, we show that a naive $\epsilon$-equilibrium always exists, for any $\epsilon>0$.

For a sophisticated decision maker, we also show that sophisticated equilibria do not always exist. We show the existence of a sophisticated 0 -equilibrium in pure strategies when payoffs are upper semicontinuous. Moreover, we show that for every $\epsilon>0$, there exists a sophisticated $\epsilon$-equilibrium.

We also examine the connection between naive and sophisticated decision makers. Let some $\epsilon>0$ be given. We show that for every sophisticated $\epsilon$-equilibrium there exists a naive $\epsilon$-equilibrium such that the 
stopping probability of every player in the sophisticated $\epsilon$-equilibrium strategy is higher than in the naive $\epsilon$-equilibrium strategy. Additionally, we show that for every naive $\epsilon$-equilibrium there exists a sophisticated $\epsilon$-equilibrium such that the stopping probability of every player in the sophisticated $\epsilon$-equilibrium strategy is higher than in the naive $\epsilon$-equilibrium. 


\section{Chapter 3}

\section{Perfect Information Games Where Each Player Acts Only Once}

\subsection{Introduction}

We study perfect information games where each player is active only once. Our emphasis is on the question of existence of subgame perfect $\epsilon$-equilibria. The references below testify to the increasing attention that the concept has been receiving in the recent game-theoretic literature. In addition to the challenging existence issue, the chapter reaches out to a large arena of research on topics such as minority games, timeinconsistent preferences, and intergenerational games.

The contribution of the chapter is twofold. The first contribution is to introduce a class of so-called frequency-based minority games. These are perfect information games where each player becomes active exactly once in the course of the game and has a choice between two actions. One is a risky action: it gives a high payoff if a minority of the players use it and a low payoff otherwise. The other is a safe action: taking this action leads to an intermediate payoff for sure, irrespective 

Once

of what the other players do. The subtlety of the construction lies in the exact meaning of the expression "a minority of the players". We allow for several specifications, the most characteristic of which invokes the frequency of the appearance of the risky action in the population. Our main result on frequency-based minority games states that these games admit no subgame perfect $\epsilon$-equilibria for small positive $\epsilon$.

Closely related to our work are two known counter-examples to existence of subgame perfect $\epsilon$-equilibrium. One is a stopping game played by a sequence of players considered in Flesch, Kuipers, MashiahYaakovi, Schoenmakers, Solan, and Vrieze (2010). The example shows that games of the type studied in this paper need not have subgame perfect $\epsilon$-equilibria in pure strategies. The other example is given in Flesch, Kuipers, Mashiah-Yaakovi, Shmaya, Schoenmakers, Solan, and Vrieze (2014). Being a game with two players, this example falls outside the class of games considered here. The authors show that the game has no subgame perfect $\epsilon$-equilibrium, whether in pure or mixed strategies. Frequency-based minority games thus demonstrate that even in games where each player acts only once, the existence of subgame perfect $\epsilon-$ equilibrium is not guaranteed.

Our second contribution is to provide several conditions for the existence of subgame perfect $\epsilon$-equilibrium. Many of these conditions have appeared previously in the literature in various contexts. We now discuss each condition in turn.

[I] Games with qualitative objectives (also called Boolean objectives): These are games where each players' payoff function takes only two values, 0 or 1 . This class is motivated by a vast literature in computer science on qualitative games. The inventory of qualitative objectives considered in the computer science literature includes reachability, safety, Büchi, parity, and Müller objectives. For the precise definitions of these objectives, we refer to the reviews by Chatterjee and Henzinger (2012) 
and Bruyère (2017). We show that games with qualitative objectives admit subgame perfect 0 -equilibria in pure strategies. The proof is based on the iterative procedure in Flesch et al. (2010).

[II] Games with upper semicontinuous payoffs. We show that games of this class admit a subgame perfect 0 -equilibrium in pure strategies. This result complements the findings of Purves and Sudderth (2011), who consider games with finitely many players.

[III] Games continuous outside a countable set. These are games in which the payoffs are continuous when restricted to a sufficiently large part of the domain. This somewhat technical condition is related to the work of Flesch and Predtetchinski (2016b). We show that this class of games admits a subgame perfect $\epsilon$-equilibrium for every positive value of $\epsilon$. The result generalizes the corresponding result of Cingiz, Flesch, Herings, and Predtetchinski (2016).

[IV] Games played by a finite number of teams. A team is a group of players with identical payoff functions. In this class of games the set of players can be partitioned into finitely many teams. The idea of teams consisting of individual players has attracted considerable attention (see e.g. von Stengel and Koller (1997), Solan (2000), Gossner and Tomala (2007), von Stengel and Zamir (2010), Gossner and Hörner (2010)). We show that games of this class admit a subgame perfect 0 -equilibrium in pure strategies.

Games where each player acts only once arise naturally in economics. One example-which we studied in the previous chapter-is decision making with time-inconsistent preferences, as modeled by a sequence of multiple selves (Strotz (1956), Pollak (1968), Peleg and Yaari (1973)). A special case of this is hyperbolic discounting (Phelps and Pollak (1968), Laibson (1994, 1997), Jaśkiewicz and Nowak (2014)). Another example relates to intergenerational games, where a player in the infinite sequence of players represents a generation (Phelps and Polak (1968), Balbus, 

Once

Jaskiewicz, Nowak (2015)). In a similar vein, Asheim (2010) considers a model with an infinite stream of generations to analyze intergenerational social welfare. A class of continuous games games played by a sequence of players is studied for example in Hellwig, Leininger, Reny, and Robson (1990). Callander and Hörner (2009) study social learning using games played by a sequence of players.

As an illustration, we consider two examples in some detail. The examples are motivated by the work of Peleg (1969) and Voorneveld (2010) on minority games. Minority games have been studied in a variety of very different contexts. For an extensive list of related work we refer to Renault, Scarsini, and Tomala (2007) and Renault, Scarlatti, and Scarsini (2008). Unlike much of the work on minority games that focusses on the setup with simultaneous moves, here we consider sequential games of perfect information.

The chapter is organized as follows. In Section 2, we provide the general model and formal definitions. In Section 3, we introduce frequencybased minority games and show that they admit no subgame perfect $\epsilon$-equilibria for small positive values of $\epsilon$. In Section 4 , we give several sufficient conditions for the existence of subgame perfect $\epsilon$-equilibrium. In Section 5, we study the two examples of perfect information games as adapted from Peleg (1969) and Voorneveld (2010).

\subsection{The model}

Preliminaries: Let $\mathbb{N}$ denote the set of natural numbers $\{1,2, \ldots\}$. Let $A$ be a non-empty finite set. For every $t \in \mathbb{N}$, let $H_{t}$ denote the set of sequences of elements of $A$ of length $t-1$. Thus, in particular, $H_{1}$ is a singleton consisting of the empty sequence ø. Let $H=\cup_{t \in \mathbb{N}} H_{t}$. Elements of $H$ are called histories. Let $A^{\mathbb{N}}$ denote the set of infinite sequences of elements of $A$. Elements of $A^{\mathbb{N}}$ are called plays. A typical play is written 
as $p=\left(a_{1}, a_{2}, \ldots\right)$. Finite sequences $\varnothing,\left(a_{1}\right),\left(a_{1}, a_{2}\right), \ldots$ are said to be prefixes of the play $\left(a_{1}, a_{2}, \ldots\right)$. A play $p$ is said to extend a history $h \in H$ if $h$ is a prefix of $p$. In a similar way, we define the prefix of a history and a history extending another history.

Given a history $h \in H$, we define the cylinder set $C(h)$ as the set of plays $p \in A^{\mathbb{N}}$ such that $h$ is a prefix of $p$. The collection of all cylinder sets is the basis of a topology on $A^{\mathbb{N}}$. In what follows, $A^{\mathbb{N}}$ is always assumed to be endowed with the topology generated by the cylinder sets. This topology is equal to the product topology, where each $A$ is given the discrete topology, and is well-known to be a Polish topology.

We let $\mathcal{B}$ denote the Borel sigma-algebra on $A^{\mathbb{N}}$, the smallest sigmaalgebra containing all the open sets. Since $A$ is assumed to be finite, $\mathcal{B}$ coincides with the sigma-algebra generated by the cylinder sets.

The game: We consider games where the set of players is equal to $\mathbb{N}$. Player $t \in \mathbb{N}$ is active in exactly one period, period $t$. In period $t$, player $t$ chooses an action $a_{t}$ from the set $A$. The chosen action is observed by all players. Depending on the infinite sequence of actions chosen $p=\left(a_{1}, a_{2}, \ldots\right)$, player $t$ receives a payoff equal to $u_{t}(p)$. Throughout the chapter we assume that, for every player $t, u_{t}$ takes only finitely many values and is Borel measurable on $A^{\mathbb{N}}$.

Let some player $t \in \mathbb{N}$ be given. A strategy $\sigma_{t}$ for player $t$ is a mapping $\sigma_{t}: H_{t} \rightarrow \Delta(A)$. Recall that $H_{t}$ is defined as the set of histories of length $t-1$, so $H_{t}$ corresponds to the set of histories where player $t$ is active. The set $\Delta(A)$ contains all probability measures on $A$. A strategy profile is denoted by $\sigma=\left(\sigma_{t}\right)_{t \in \mathbb{N}}$. The set of strategies for player $t \in \mathbb{N}$ is denoted by $\Sigma_{t}$ and the set of strategy profiles by $\Sigma=\times_{t \in \mathbb{N}} \Sigma_{t}$.

A strategy $\sigma_{t}$ for player $t \in \mathbb{N}$ is called pure if for every $h \in H_{t}, \sigma_{t}(h)$ places all the probability on a single action. The set of pure strategies for player $t$ is denoted by $S_{t}$. A pure strategy $s_{t} \in S_{t}$ is treated as a function $s_{t}: H_{t} \rightarrow A$. The set of pure strategy profiles is denoted by $S=\times_{t \in \mathbb{N}} S_{t}$. 
We define $\rho: S \times H \rightarrow A^{\mathbb{N}}$ by letting $\rho(s \mid h)$ denote the play induced by the pure strategy profile $s$ conditional on reaching history $h$. Formally, for every $t \in \mathbb{N}$, for every $h \in H_{t}$, we let $\rho(s \mid h)$ be the play $\left(h, a_{t}, a_{t+1}, \ldots\right)$ where $a_{t}=s_{t}(h), a_{t+1}=s_{t+1}\left(h, a_{t}\right)$, and so on. The payoff for a player $t \in \mathbb{N}$ induced by a pure strategy profile $s \in S$ at $h \in H_{t}$ is denoted by $v_{t}(s \mid h)=u_{t}(\rho(s \mid h))$.

Given a strategy profile $\sigma \in \Sigma$ and a history $h \in H$, we let $\mu_{\sigma, h}$ denote the Borel probability measure on $A^{\mathbb{N}}$ induced by $\sigma$ in the subgame starting with history $h$. Thus, $\mu_{\sigma, h}$ places probability 1 on the cylinder set $C(h)$. The expected payoff for a player $t \in \mathbb{N}$, induced by $\sigma$ at $h$, is equal to

$$
v_{t}(\sigma \mid h)=\mathbb{E}_{\mu_{\sigma, h}}\left(u_{t}\right)=\int_{A^{\mathbb{N}}} u_{t} \mathrm{~d} \mu_{\sigma, h} .
$$

We denote $v_{t}(\sigma \mid \varnothing)$ by $v_{t}(\sigma)$. This brings us to the following definitions.

$\epsilon$-Equilibrium: For $\epsilon \geq 0$, a strategy profile $\sigma \in \Sigma$ is an $\epsilon$-equilibrium if for every player $t \in \mathbb{N}$, for every strategy $\sigma_{t}^{\prime} \in \Sigma_{t}$,

$$
v_{t}(\sigma) \geq v_{t}\left(\sigma_{-t}, \sigma_{t}^{\prime}\right)-\epsilon
$$

Mertens and Neyman (see Mertens (1987)) have shown that, for any game satisfying our assumptions, a pure strategy 0 -equilibrium exists. ${ }^{1}$

A strategy profile that constitutes an $\epsilon$-equilibrium for a game does not necessarily induce an $\epsilon$-equilibrium in every subgame, even though that would be highly desirable for a solution concept. This is the main motivation for considering the following refinement of $\epsilon$-equilibrium.

Subgame perfect $\epsilon$-equilibrium: For $\epsilon \geq 0$, a strategy profile $\sigma \in \Sigma$ is a subgame perfect $\epsilon$-equilibrium if for every history $h \in H$, for every player $t \in \mathbb{N}$, for every strategy $\sigma_{t}^{\prime} \in \Sigma_{t}$,

$$
v_{t}(\sigma \mid h) \geq v_{t}\left(\sigma_{-t}, \sigma_{t}^{\prime} \mid h\right)-\epsilon .
$$

\footnotetext{
${ }^{1}$ The argument is given for games with finitely many players. It is clear that it remains valid in the present setting where the number of players is infinite.
} 
Thus a strategy profile $\sigma \in \Sigma$ is a subgame perfect $\epsilon$-equilibrium if and only if, for every $h \in H, \sigma$ induces an $\epsilon$-equilibrium in the subgame starting with history $h$ if and only if for every $t \in \mathbb{N}$, for every $h \in H_{t}$, for every $a_{t} \in A$,

$$
v_{t}(\sigma \mid h) \geq v_{t}\left(\sigma_{-t}, a_{t} \mid h\right)-\epsilon .
$$

The main emphasis of the chapter is to study the existence of subgame perfect $\epsilon$-equilibrium in the class of games satisfying our assumptions.

\subsection{Frequency-based minority games}

This section is devoted to a class of games that do not have a subgame perfect $\epsilon$-equilibrium for small positive $\epsilon$. It is divided into three subsections. The first subsection presents the leading example and a definition of frequency-based minority games. The second subsection contains additional examples and remarks on this class. In the third subsection we state our main result, and the fourth subsection contains the proof.

\subsubsection{The leading example and the definition}

Before we give a definition of the class of frequency-based minority games, we start with an example.

Example 3.3.1. Consider a game where the action set is given by $A=$ $\{0,1\}$. For $t \in \mathbb{N}$, player $t$ 's payoff function is given by

$$
u_{t}\left(a_{1}, a_{2}, \ldots\right)= \begin{cases}1, & \text { if } a_{t}=1, \\ 2, & \text { if } a_{t}=0 \text { and }\left(a_{1}, a_{2}, \ldots\right) \in F \\ 0, & \text { if } a_{t}=0 \text { and }\left(a_{1}, a_{2}, \ldots\right) \in A^{\mathbb{N}} \backslash F,\end{cases}
$$

where

$$
F=\left\{\left(a_{1}, a_{2}, \ldots\right) \in A^{\mathbb{N}}: \limsup _{t \rightarrow \infty} \frac{1}{t}\left(a_{1}+\cdots+a_{t}\right)>\frac{1}{2}\right\}
$$


Action 1 is a safe action that leads to a payoff of 1 irrespective of the actions chosen by the other players. Action 0 is a risky action. It leads to a payoff of 2 if the play of the game belongs to the set $F$ and to a payoff of 0 otherwise, so the risky action pays off if it is chosen by a minority of the players. The set $F$ expresses the condition that only a minority of the players use action 0 . More precisely, $F$ consists of the plays such that the limsup frequency of action 1 exceeds $1 / 2$.

As follows from our main result, the game in Example 3.3.1 has no subgame perfect $\epsilon$-equilibrium for any $\epsilon$ sufficiently small. Rather than proving this result specifically for the game of Example 3.3.1, we identify a whole class of games where the same result applies. These are the so-called frequency-based minority games.

We say that a set $F \subset A^{\mathbb{N}}$ is admissible if

[F1] $F$ is a Borel subset of $A^{\mathbb{N}}$.

[F2] $F$ is a tail set in the following sense: if $\left(a_{1}, a_{2}, \ldots\right) \in F$ and $\left(a_{1}^{\prime}, a_{2}^{\prime}, \ldots\right) \in$ $A^{\mathbb{N}}$ is such that $a_{n}^{\prime} \neq a_{n}$ for at most finitely many $n \in \mathbb{N}$, then $\left(a_{1}^{\prime}, a_{2}^{\prime}, \ldots\right) \in F$.

[F3] $F$ is closed under $\leq:$ if $\left(a_{1}, a_{2}, \ldots\right) \in F$ and $\left(a_{1}^{\prime}, a_{2}^{\prime}, \ldots\right) \in A^{\mathbb{N}}$ is such that, for every $t \in \mathbb{N}, a_{t} \leq a_{t}^{\prime}$, then $\left(a_{1}^{\prime}, a_{2}^{\prime}, \ldots\right) \in F$.

[F4] Let $\beta_{1}, \beta_{2}, \ldots$ be a sequence of i.i.d. random variables taking values in $\{0,1\}$. There exists $v^{*} \in(0,1)$ such that if each $\beta_{t}$ is equal to 1 with probability $v^{*}$, then

$$
\mathbb{P}\left(\left(\beta_{1}, \beta_{2}, \ldots\right) \in F\right)=1 .
$$

[F5] Let $\beta_{1}^{\prime}, \beta_{2}^{\prime}, \ldots$ be a sequence of i.i.d. random variables taking values in $\{0,1\}$. There exists $v_{*} \in(0,1)$ such that if each $\beta_{t}^{\prime}$ is equal to 1 with probability $v_{*}$, then

$$
\mathbb{P}\left(\left(\beta_{1}^{\prime}, \beta_{2}^{\prime}, \ldots\right) \in F\right)=0 .
$$


The set $F$ describes the plays being such that action 0 is played by a minority of the players. Condition [F1] is a technical measurability condition. By Condition [F2], whether a play belongs to $F$ or not depends only on the tail of the play. Condition [F3] expresses that if the set of players choosing action 1 at some play that belongs to $F$ is a subset of the set of players choosing action 1 at another play, then the other play belongs to $F$ as well. By Condition [F4] it holds that if all players choose action 1 independently with sufficiently high probability, then the play belongs to $F$ with probability 1 . Similarly, by Condition [F5], if all players choose action 1 independently with sufficiently low probability, then the play belongs to $F$ with probability 0 .

Definition 3.3.2. A game is a frequency-based minority game if $A=\{0,1\}$, the set $F$ is admissible, and, for every $t \in \mathbb{N}$, player $t^{\prime}$ s payoff function is given by equation (3.3).

\subsubsection{Further discussion on admissible sets}

It is easily verified that the set $F$ in Example 3.3.1 is admissible, so the game in Example 3.3.1 is a frequency-based minority game. In particular it satisfies condition [F4] with for instance $v^{*}=2 / 3$ and condition [F5] with $v_{*}=1 / 3$ as a consequence of the law of large numbers. The choice of $1 / 2$ as a lower bound on the frequency of action $1 \mathrm{in}(3.4)$ is inessential. Any number in the open interval $(0,1)$ yields a set $F$ that is admissible. Likewise the set

$$
\left\{\left(a_{1}, a_{2}, \ldots\right) \in A^{\mathbb{N}}: \liminf _{t \rightarrow \infty} \frac{1}{t}\left(a_{1}+\cdots+a_{t}\right)>\frac{1}{2}\right\}
$$

is admissible. A somewhat less obvious example of an admissible set is

$$
\left\{\left(a_{1}, a_{2}, \ldots\right) \in A^{\mathbb{N}}: \ell\left(a_{1}, a_{2}, \ldots\right)>\frac{1}{2}\right\},
$$

where $\ell$ is a medial limit, a special type of Banach limit, as described in Mertens, Sorin and Zamir (2015, page 29). This medial limit $\ell$ is a 
positive linear functional that assigns a real number to every bounded sequence of real numbers, and has various appealing properties. For example, for every $\left(a_{1}, a_{2}, \ldots\right) \in A^{\mathbb{N}}$ it holds that

$$
\begin{aligned}
& \ell\left(a_{1}, a_{2}, \ldots\right)=\ell\left(a_{1}, \frac{1}{2}\left(a_{1}+a_{2}\right), \frac{1}{3}\left(a_{1}+a_{2}+a_{3}\right), \ldots\right), \\
& \ell\left(a_{1}, a_{2}, a_{3}, \ldots\right)=\ell\left(a_{2}, a_{3}, \ldots\right), \\
& \liminf _{t \rightarrow \infty} \frac{1}{t}\left(a_{1}+\cdots+a_{t}\right) \leq \ell\left(a_{1}, a_{2}, \ldots\right) \leq \limsup _{t \rightarrow \infty} \frac{1}{t}\left(a_{1}+\cdots+a_{t}\right) .
\end{aligned}
$$

It is easy to make use of examples of admissible sets to create new examples, since the collection of admissible sets is closed under finite unions and finite intersections.

It follows from the topological zero-one law (Kechris [1995], Theorem 8.47) that an admissible set is either meagre or comeagre. The Hewitt-Savage zero-one law (Shiryaev [1996], page 382) implies that if $F$ is admissible and $\beta_{1}, \beta_{2}, \ldots$ is an i.i.d. sequence of Bernoulli random variables, then the probability $\mathbb{P}\left(\left(\beta_{1}, \beta_{2}, \ldots\right) \in F\right)$ is either 0 or 1 .

Next we remark that any admissible set is sandwiched between the sets $E$ and $E^{\prime}$ defined as follows:

$$
\begin{aligned}
E & =\left\{\left(a_{1}, a_{2}, \ldots\right) \in A^{\mathbb{N}} \mid\left\{t \in \mathbb{N}: a_{t}=0\right\} \text { is finite }\right\} . \\
E^{\prime} & =\left\{\left(a_{1}, a_{2}, \ldots\right) \in A^{\mathbb{N}} \mid\left\{t \in \mathbb{N}: a_{t}=1\right\} \text { is infinite }\right\} .
\end{aligned}
$$

Claim 3.3.3. If $F$ is an admissible set, then $E \subset F \subset E^{\prime}$.

Proof. The set $F$ is non-empty by [F4]. By [F3] it must contain the play $(1,1, \ldots)$. It now follows by [F2] that it contains all plays in $E$.

Suppose that $F$ is not a subset of $E^{\prime}$. Then $F$ contains a play $\left(a_{1}, a_{2}, \ldots\right)$ such that only finitely many players choose action 1 . Replacing the finitely many 1's by 0 's and making use of [F2], we conclude that $F$ contains the play $(0,0, \ldots)$. But then, by $[\mathrm{F} 3]$, we have $F=A^{\mathbb{N}}$, contradicting [F5]. 
The sets $E$ and $E^{\prime}$ are not admissible. More precisely, the set $E$ satisfies [F1], [F2], [F3] and [F5], but not [F4]. Indeed, if $\beta_{1}, \beta_{2}, \ldots$ is a sequence of i.i.d. distributed random variables, each taking value 1 with probability $v>0$, then with probability 1 the realization of infinitely many random variables $\beta_{t}$ is equal to 0 . The set $E^{\prime}$ is not admissible either. Indeed, $E^{\prime}$ satisfies [F1], [F2], [F3] [F4], but not [F5].

\subsubsection{The main result and overview of the proof}

The main result of this section is the following theorem.

Theorem 3.3.4. Let $G$ be a frequency-based minority game with admissible set F. Let $v^{*}$ be as in [F4] and $v_{*}$ as in [F5]. Then $G$ has no subgame perfect $\epsilon$-equilibrium whenever $\epsilon<\min \left\{v_{*},\left(1-v^{*}\right) / 3\right\}$.

It is easy to see that $G$ has no subgame perfect 0 -equilibrium in pure strategies. Indeed, suppose that $\sigma$ is a pure subgame perfect 0 equilibrium of $G$. Then after each history, the play induced by $\sigma$ lies in $F$. This is because each player can guarantee a payoff of 1 by taking the safe action 1. If the induced play would not be in $F$, then the players taking action 0 would be receiving a payoff of 0 , contradicting the fact that $\sigma$ is subgame perfect 0 -equilibrium. But then, since the continuation play after any history is an element of $F$, every player prefers to choose action 0 , leading to a contradiction.

The difficulty of Theorem 3.3.4 lies in working with mixed strategies. A formal proof of the theorem is given in the next subsection. Here we present an intuitive and a deliberately non-rigorous account of the proof.

We will argue that if $\sigma$ is a subgame perfect $\epsilon$-equilibrium, then for each history $h$ with $\mu_{\sigma, h}(F)<\epsilon$, the active player takes action 1 with a probability of at least $1-3 \epsilon$ (this is Step 2 below). Very roughly speaking, this implies that, as soon as the game reaches a stage where it becomes "unlikely" that action 1 is chosen by the majority of the players, 
the players start using action 1 with a very "high" probability. It is intuitively clear that no strategy profile can satisfy this condition. The formal argument invokes coupling.

Coupling is a technique from probability theory, which is useful whenever the distributions of two distinct random variables need to be compared to each other. The technique consists of creating a new, larger probability space, and two new random variables, having the same distributions as the two original ones, with some additional property that affords a simpler comparison of the two.

We couple two processes: one is the process $\alpha_{1}, \alpha_{2}, \ldots$ on $A$ governed by the strategy profile $\sigma$, the other one is the process $\beta_{1}, \beta_{2}, \ldots$ of i.i.d. Bernoulli random variables, each taking the value 1 with probability $1-3 \epsilon$. We couple the process so that $\alpha_{t} \geq \beta_{t}$ whenever at period $t$ the conditional probability of $F$ drops below $\epsilon$ (this is our Step 3 ). The advantage of having this coupling is that by [F4], the probability distribution of $\beta_{1}, \beta_{2}, \ldots$ assigns probability 1 to the set $F$. Using Lévy's zero-one law this allows us to conclude that also the distribution of $\alpha_{1}, \alpha_{2}, \ldots$ assigns probability 1 to $F$ (Step 4). This quickly leads to a contradiction.

\subsubsection{The proof of Theorem 3.3.4}

To prove the main result, we adopt the following notational convention. Given a player $t \in \mathbb{N}$, a history $h \in H_{t}$, and a strategy profile $\sigma$, we use $\sigma_{t}(h)$ to denote the probability that $\sigma_{t}(h)$ places on action 1 . The probability that the resulting play belongs to $F$ is denoted by $\tau(\sigma \mid h)$, so

$$
\tau(\sigma \mid h)=\mu_{\sigma, h}(F)
$$

We employ coupling twice, in Steps 1 and 3, but it is only essential in Step 3. The use of coupling in Step 1 could be replaced by an appeal to a much more general Theorem 5.8 in Lindvall [1992]. We choose to give 
an explicit construction of the coupling because it is very similar to the construction employed in Step 3.

SteP 1: Let $\sigma \in \Sigma$ be a strategy profile. If, for every $t \in \mathbb{N}$, for every $h \in H_{t}, \sigma_{t}(h) \geq v^{*}$, then $\tau(\sigma \mid \varnothing)=1$. If, for every $t \in \mathbb{N}$, for every $h \in H_{t}$, $\sigma_{t}(h) \leq v_{*}$, then $\tau(\sigma \mid \varnothing)=0$.

ProOf Of STEP 1: We prove the first statement. The proof of the second statement is similar.

We first construct a probability space $(\Omega, \mathcal{S}, \mathbb{P})$ and, for every $t \in \mathbb{N}$, random variables $\alpha_{t}: \Omega \rightarrow\{0,1\}$ and $\beta_{t}: \Omega \rightarrow\{0,1\}$ satisfying the following three properties:

1. For every $t \in \mathbb{N}$, for every $\left(a_{1}, \ldots, a_{t-1}\right) \in H_{t}$,

$$
\mathbb{P}\left(\alpha_{t}=1 \mid \alpha_{1}=a_{1}, \ldots, \alpha_{t-1}=a_{t-1}\right)=\sigma_{t}\left(a_{1}, \ldots, a_{t-1}\right) .
$$

2. The random variables $\beta_{1}, \beta_{2}, \ldots$ are i.i.d. with, for every $t \in \mathbb{N}$, $\mathbb{P}\left(\beta_{t}=1\right)=v^{*}$.

3. For every $t \in \mathbb{N}, \alpha_{t} \geq \beta_{t}$.

Let $B=\{0,1\}$ and consider a game as in Section 3.2 where the action set is equal to $A \times B=\{0,1\} \times\{0,1\}$. We define the strategy profile $\lambda: \times_{t \in \mathbb{N}}(A \times B)^{t-1} \rightarrow \Delta(A \times B)$ as follows. For every player $t \in \mathbb{N}$, for every history $\left(a_{1}, b_{1}, \ldots, a_{t-1}, b_{t-1}\right) \in(A \times B)^{t-1}$, for every action $\left(a_{t}, b_{t}\right) \in A \times B$, the value of $\lambda_{t}\left(a_{1}, b_{1}, \ldots, a_{t-1}, b_{t-1}\right)\left(a_{t}, b_{t}\right)$ is as given in Table 1.

Let $\Omega$ be the set $(A \times B)^{\mathbb{N}}, \mathcal{S}$ the sigma-algebra of Borel sets of $\Omega$, and $\mathbb{P}$ the probability measure $\mu_{\lambda, \varnothing}$. For every $t \in \mathbb{N}$, let $\alpha_{t}$ and $\beta_{t}$ be the random variables defined by $\alpha_{t}\left(a_{1}, b_{1}, a_{2}, b_{2}, \ldots\right)=a_{t}$ and $\beta_{t}\left(a_{1}, b_{1}, a_{2}, b_{2}, \ldots\right)=b_{t}$. It is easy to check that $\alpha_{t}$ and $\beta_{t}$ have the three desired properties. 
Chapter 3. Perfect Information Games Where Each Player Acts Only Once

\begin{tabular}{|c|cc|}
\hline & $b_{t}=0$ & $b_{t}=1$ \\
\hline$a_{t}=0$ & $1-\sigma_{t}(h)$ & 0 \\
$a_{t}=1$ & $\sigma_{t}(h)-v^{*}$ & $v^{*}$ \\
\hline
\end{tabular}

Table 3.1: The value of $\lambda_{t}\left(a_{1}, b_{1}, \ldots, a_{t-1}, b_{t-1}\right)\left(a_{t}, b_{t}\right)$, where $h=\left(a_{1}, \ldots, a_{t-1}\right)$.

The sequence $\left(\alpha_{1}, \alpha_{2}, \ldots\right)$ induces the probability measure $\mu_{\sigma, \varnothing}$ on the set of plays $A^{\mathbb{N}}$. It follows that

$$
1=\mathbb{P}\left(\left(\beta_{1}, \beta_{2}, \ldots\right) \in F\right) \leq \mathbb{P}\left(\left(\alpha_{1}, \alpha_{2}, \ldots\right) \in F\right)=\mu_{\sigma, \varnothing}(F)=\tau(\sigma \mid \varnothing),
$$

where the first equality follows from [F4] and the inequality from [F3].

Now choose $\epsilon>0$ such that $\epsilon<v_{*}$ and $1-3 \epsilon>v^{*}$.

Suppose $\sigma \in \Sigma$ is a subgame perfect $\epsilon$-equilibrium of $G$.

STEP 2: For every $t \in \mathbb{N}$, for every $h \in H_{t}$ such that $\tau(\sigma \mid h)<\epsilon$, it holds that $\sigma_{t}(h) \geq 1-3 \epsilon$.

Proof OF SteP 2: Let some $t \in \mathbb{N}$ and some $h \in H_{t}$ be given. It holds that

$$
\tau(\sigma \mid h)=\sigma_{t}(h) \cdot \tau(\sigma \mid h, 1)+\left(1-\sigma_{t}(h)\right) \cdot \tau(\sigma \mid h, 0)
$$

or, equivalently,

$$
\left(1-\sigma_{t}(h)\right) \cdot \tau(\sigma \mid h, 0)=\tau(\sigma \mid h)-\sigma_{t}(h) \cdot \tau(\sigma \mid h, 1) .
$$

Now player $t^{\prime}$ s expected payoff at history $h$ is

$$
v_{t}(\sigma \mid h)=\sigma_{t}(h)+2 \cdot\left(1-\sigma_{t}(h)\right) \cdot \tau(\sigma \mid h, 0) .
$$

Plugging equation (3.5) into equation (3.6), we get

$$
v_{t}(\sigma \mid h)=\sigma_{t}(h)+2 \cdot\left(\tau(\sigma \mid h)-\sigma_{t}(h) \cdot \tau(\sigma \mid h, 1)\right) .
$$


Since playing action 1 yields a payoff of 1 and since $\sigma$ is a subgame perfect $\epsilon$-equilibrium, we have $v_{t}(\sigma \mid h) \geq 1-\epsilon$. It follows that

$1-\epsilon \leq v_{t}(\sigma \mid h)=\sigma_{t}(h)+2 \cdot\left(\tau(\sigma \mid h)-\sigma_{t}(h) \cdot \tau(\sigma \mid h, 1)\right) \leq \sigma_{t}(h)+2 \cdot \tau(\sigma \mid h)$.

This implies the statement of Step 2.

STEP 3: Definition of appropriate random variables $\alpha_{1}, \alpha_{2}, \ldots$ and $\beta_{1}, \beta_{2}, \ldots$

We construct a probability space $(\Omega, \mathcal{S}, \mathbb{P})$ and, for every $t \in \mathbb{N}$, random variables $\alpha_{t}: \Omega \rightarrow\{0,1\}$ and $\beta_{t}: \Omega \rightarrow\{0,1\}$ satisfying the following three properties:

1. For every $t \in \mathbb{N}$, for every $\left(a_{1}, \ldots, a_{t-1}\right) \in H_{t}$,

$$
\mathbb{P}\left(\alpha_{t}=1 \mid \alpha_{1}=a_{1}, \ldots, \alpha_{t-1}=a_{t-1}\right)=\sigma_{t}\left(a_{1}, \ldots, a_{t-1}\right) .
$$

2. The random variables $\beta_{1}, \beta_{2}, \ldots$ are i.i.d. with, for every $t \in \mathbb{N}$, $\mathbb{P}\left(\beta_{t}=1\right)=1-3 \epsilon$.

3. For every $t \in \mathbb{N}$, for every $\left(a_{1}, \ldots, a_{t-1}\right) \in H_{t}, \tau\left(\sigma \mid a_{1}, \ldots, a_{t-1}\right)<\epsilon$ implies $\alpha_{t} \geq \beta_{t}$.

Let $B=\{0,1\}$ and consider a game as in Section 3.2 where the action set is equal to $A \times B=\{0,1\} \times\{0,1\}$. We define the strategy profile $\lambda: \times_{t \in \mathbb{N}}(A \times B)^{t-1} \rightarrow \Delta(A \times B)$ as follows. For every player $t \in \mathbb{N}$, for every history $\left(a_{1}, b_{1}, \ldots, a_{t-1}, b_{t-1}\right) \in(A \times B)^{t-1}$, for every action $\left(a_{t}, b_{t}\right) \in A \times B$, the value of $\lambda_{t}\left(a_{1}, b_{1}, \ldots, a_{t-1}, b_{t-1}\right)\left(a_{t}, b_{t}\right)$ is as given in Table 2.

Notice that the probability that $\left(a_{t}, b_{t}\right)=(1,0)$ when $\tau(\sigma \mid h)<\epsilon$ is equal to $\sigma_{t}(h)-(1-3 \epsilon)$, which is non-negative by Step 2 .

Let $\Omega$ be the set $(A \times B)^{\mathbb{N}}, \mathcal{S}$ the sigma-algebra of Borel sets of $\Omega$, and $\mathbb{P}$ the probability measure $\mu_{\lambda, \varnothing}$. For every $t \in \mathbb{N}$, let $\alpha_{t}$ and $\beta_{t}$ be the random variables defined by $\alpha_{t}\left(a_{1}, b_{1}, a_{2}, b_{2}, \ldots\right)=a_{t}$ and 
Chapter 3. Perfect Information Games Where Each Player Acts Only Once

\begin{tabular}{|c|c|c|c|c|}
\hline & \multicolumn{2}{|c|}{$b_{t}=0$} & \multicolumn{2}{|c|}{$b_{t}=1$} \\
\hline$a_{t}=0$ & \multicolumn{2}{|c|}{$3 \epsilon \cdot\left(1-\sigma_{t}(h)\right)$} & \multicolumn{2}{|c|}{$(1-3 \epsilon) \cdot\left(1-\sigma_{t}(h)\right.$} \\
\hline$a_{t}=1$ & \multicolumn{2}{|c|}{$3 \epsilon \cdot \sigma_{t}(h)$} & \multicolumn{2}{|c|}{$(1-3 \epsilon) \cdot \sigma_{t}(h)$} \\
\hline & \multicolumn{4}{|c|}{$\tau(\sigma \mid h)<\epsilon$} \\
\hline & & & $=0$ & $b_{t}=1$ \\
\hline & $a_{t}=0$ & $1-$ & $\sigma_{t}(h)$ & 0 \\
\hline & $a_{t}=1$ & $\sigma_{t}(h)-$ & $(1-3 \epsilon)$ & $1-3 \epsilon$ \\
\hline
\end{tabular}

Table 3.2: The value of $\lambda_{t}\left(a_{1}, b_{1}, \ldots, a_{t-1}, b_{t-1}\right)\left(a_{t}, b_{t}\right)$, where $h=\left(a_{1}, \ldots, a_{t-1}\right)$.

$\beta_{t}\left(a_{1}, b_{1}, a_{2}, b_{2}, \ldots\right)=b_{t}$. It is easy to check that $\alpha_{t}$ and $\beta_{t}$ have the three desired properties. This completes the definition of the sequences.

Property 1 , the fact that $1-3 \epsilon>v^{*}$, and Step 1 imply that

$$
\mathbb{P}\left(\left(\beta_{1}, \beta_{2}, \ldots\right) \in F\right)=1 \text {. }
$$

Furthermore, the sequence $\left(\alpha_{1}, \alpha_{2}, \ldots\right)$ induces the probability measure $\mu_{\sigma, \varnothing}$ on the set of plays $A^{\mathbb{N}}$. It follows that

$$
\mathbb{P}\left(\left(\alpha_{1}, \alpha_{2}, \ldots\right) \in F\right)=\mu_{\sigma, \varnothing}(F)=\tau(\sigma \mid \varnothing) .
$$

STEP 4: $\tau(\sigma \mid \varnothing)=1$.

ProOf OF Step 4: For every $t \in \mathbb{N}$, define the random variable $\psi_{t}: \Omega \rightarrow$ $\{0,1\}$ by letting $\psi_{t}\left(a_{1}, b_{1}, a_{2}, b_{2}, \ldots\right)=1$ if $\tau\left(\sigma \mid a_{1}, \ldots, a_{t-1}\right)<\epsilon$ and $\psi_{t}\left(a_{1}, b_{1}, a_{2}, b_{2}, \ldots\right)=0$, otherwise. Notice that $\psi_{t}=1$ implies $\beta_{t} \leq \alpha_{t}$. Define the random variable $\psi: \Omega \rightarrow A$ by $\psi\left(a_{1}, b_{1}, a_{2}, b_{2}, \ldots\right)=1$ if $\left(a_{1}, a_{2}, \ldots\right) \in F^{\mathrm{C}}$ and $\psi\left(a_{1}, b_{1}, a_{2}, b_{2}, \ldots\right)=0$ if $\left(a_{1}, a_{2}, \ldots\right) \in F$. Notice that by definition $\mathbb{P}\left(\left(\alpha_{1}, \alpha_{2}, \ldots\right) \in F^{\mathrm{C}}\right)=\mathbb{P}(\psi=1)$. 
We argue that $\psi_{t}$ converges $\mathbb{P}$-almost surely to $\psi$. To see this, let $1_{F}: A^{\mathbb{N}} \rightarrow\{0,1\}$ be the indicator function of the set $F$ and let $C$ be the set of plays $\left(a_{1}, a_{2}, \ldots\right) \in A^{\mathbb{N}}$ such that $\tau\left(\sigma \mid a_{1}, \ldots, a_{t}\right)$ converges to $1_{F}\left(a_{1}, a_{2}, \ldots\right)$ as $t$ approaches infinity. Applying Lévy's zero-one law to the set $F$, we conclude that $\mu_{\sigma, \varnothing}(C)=1 .^{2}$ Now we have

$$
\mathbb{P}\left(\psi_{t} \rightarrow \psi\right) \geq \mathbb{P}\left(\left(\alpha_{1}, \alpha_{2}, \ldots\right) \in C\right)=\mu_{\sigma, \varnothing}(C)=1,
$$

where the first equality follows because the sequence $\left(\alpha_{1}, \alpha_{2}, \ldots\right)$ induces the probability measure $\mu_{\sigma, \varnothing}$ on $A^{\mathbb{N}}$.

Combining these facts we obtain the following chain of equalities and inequalities:

$$
\begin{aligned}
& \mathbb{P}\left(\left(\alpha_{1}, \alpha_{2}, \ldots\right) \in F^{\mathrm{c}}\right) \\
& =\mathbb{P}(\psi=1) \\
& =\mathbb{P}\left(\left(\beta_{1}, \beta_{2}, \ldots\right) \in F \text { and } \psi=1\right) \\
& =\mathbb{P}\left(\exists m \in \mathbb{N} \text { s.t. }\left(\beta_{1}, \beta_{2}, \ldots\right) \in F \text { and } \psi_{m}=\psi_{m+1}=\cdots=1\right) \\
& \leq \mathbb{P}\left(\exists m \in \mathbb{N} \text { s.t. }\left(\alpha_{1}, \alpha_{2}, \ldots\right) \in F \text { and } \psi_{m}=\psi_{m+1}=\cdots=1\right) \\
& =\mathbb{P}\left(\left(\alpha_{1}, \alpha_{2}, \ldots\right) \in F \text { and } \psi=1\right) \\
& =\mathbb{P}(\psi=0 \text { and } \psi=1) \\
& =0,
\end{aligned}
$$

where (3.8) holds by definition of $\psi,(3.9)$ is true because of (3.7), and equation (3.10) is true because $\psi_{t}$ converges $\mathbb{P}$-almost surely to $\psi$. To prove inequality (3.11), suppose that $\left(\beta_{1}, \beta_{2}, \ldots\right) \in F$ and $\psi_{m}=\psi_{m+1}=$ $\cdots=1$. By construction, we have that $\beta_{t} \leq \alpha_{t}$ whenever $\psi_{t}=1$. Since $F$ is closed under $\leq$ by [F3] we conclude that the vector $\left(\beta_{1}, \ldots, \beta_{m-1}\right.$, $\left.\alpha_{m}, \alpha_{m+1}, \ldots\right)$ is in $F$. Now using the tail property [F2] we can replace the finite prefix $\left(\beta_{1}, \ldots, \beta_{m-1}\right)$ by $\left(\alpha_{1}, \ldots, \alpha_{m-1}\right)$ and conclude that $\left(\alpha_{1}, \alpha_{2}, \ldots\right)$

\footnotetext{
${ }^{2}$ See Appendix A for a precise statement of Lévy's zero-one law.
} 
Chapter 3. Perfect Information Games Where Each Player Acts Only Once

is in F. Inequality (3.11) follows. Finally (3.12) holds because $\psi_{t}$ converges $\mathbb{P}$-almost surely to $\psi$ and (3.13) holds by definition of $\psi$.

STEP 5: For every $t \in \mathbb{N}$, for every $h \in H_{t}, \tau(\sigma \mid h)=1$.

Proof Of Step 5: Let some $t \in \mathbb{N}$ and some $h \in H_{t}$ be given. We define

$$
F^{\prime}=\left\{\left(a_{1}, a_{2}, \ldots\right) \in A^{\mathbb{N}}:\left(h, a_{1}, a_{2}, \ldots\right) \in F\right\} .
$$

It is not difficult to show that $F^{\prime}$ is an admissible set. In particular, it holds that $\mathbb{P}\left(\left(\beta_{1}, \beta_{2}, \ldots\right) \in F\right)=\mathbb{P}\left(\left(\beta_{1}, \beta_{2}, \ldots\right) \in F^{\prime}\right)$ for any sequence $\left(\beta_{1}, \beta_{2}, \ldots\right)$ of i.i.d. Bernoulli random variables. This implies that $F^{\prime}$ satisfies [F4] and [F5] as we can take $v^{* \prime}=v^{*}$ and $v_{*}^{\prime}=v_{*}$.

Let $G^{\prime}$ be the frequency-based minority game corresponding to $F^{\prime}$. Define the strategy profile $\sigma^{\prime} \in \Sigma$ by letting $\sigma_{t^{\prime}}\left(h^{\prime}\right)=\sigma_{t+t^{\prime}-1}\left(h, h^{\prime}\right)$ for every $t^{\prime} \in \mathbb{N}$ and $h^{\prime} \in H_{t^{\prime}}$. Then $\sigma^{\prime}$ is a subgame perfect $\epsilon$-equilibrium of $G^{\prime}$. Hence Step 4 implies that $\mu_{\sigma^{\prime}, \varnothing}\left(F^{\prime}\right)=1$. The result of Step 5 now follows since $\mu_{\sigma, h}(F)=\mu_{\sigma^{\prime}, \varnothing}\left(F^{\prime}\right)$.

STEP 6: The game G has no subgame perfect $\epsilon$-equilibrium.

ProOF OF STEP 6: Let some $t \in \mathbb{N}$ and some $h \in H_{t}$ be given. Since $\tau(\sigma \mid h, 0)=1$ by Step 5 , action 0 yields player $t$ a payoff of 2 and action 1 a payoff of 1 . It follows that $\sigma_{t}(h) \leq \epsilon<v_{*}$. By Step 1, we have $\tau(\sigma \mid \varnothing)=0$, yielding a contradiction to the statement of Step 4 .

\subsection{Sufficient conditions for existence}

For games with finitely many players, a number of sufficient conditions for the existence of subgame perfect $\epsilon$-equilibrium have been identified, see for instance Kuipers, Flesch, Schoenmakers, and Vrieze (2016) and Flesch and Predtetchinski (2016a, 2016b). Much less is known about games with infinitely many players.

Below we discuss four classes of games for which subgame perfect $\epsilon$-equilibria exist: games with qualitative objectives, games with upper 
semicontinuous payoffs, games continuous outside a countable set, and games played by a finite number of teams. Many of the conditions that we discuss have previously appeared in the literature in various contexts.

Throughout Section 3.4, we fix a game G satisfying the assumptions made in Section 3.2.

\subsubsection{Games with qualitative objectives}

The game $G$ is said to have qualitative objectives if for each player $t \in \mathbb{N}$ the payoff function $u_{t}$ takes at most two values, 0 and 1. Example 3.5.1 in the next section is a game with qualitative payoffs. The literature on computer science provides many examples of qualitative objectives. Examples are the objectives of reachability and safety and the criteria of Büchi, parity, and Müller, see the reviews by Chatterjee and Henzinger (2012) and Bruyère (2017). For $t \in \mathbb{N}$, we let $W_{t}$ denote the set of plays $p \in A^{\mathbb{N}}$ for which $u_{t}(p)=1$. We think of $W_{t}$ as the winning set of player $t$.

Theorem 3.4.1. If the game $G$ has qualitative objectives, then it has a subgame perfect 0 -equilibrium in pure strategies.

We apply the iterative algorithm developed in Flesch et al. (2010) to prove Theorem 3.4.1. For each ordinal number $\xi$, we recursively define the collection $\left\{P_{\xi}(h): h \in H\right\}$ of sets of plays and the collection $\left\{\alpha_{\xi}(h): h \in H\right\}$ of real numbers augmented with $+\infty$. For ordinal number 0 , for every $t \in \mathbb{N}$, for every history $h \in H_{t}$, we define

$$
\begin{gathered}
P_{0}(h)=\left\{p \in A^{\mathbb{N}}: h \text { is a prefix of } p\right\}, \\
\alpha_{0}(h)=\min _{p \in P_{0}(h)} u_{t}(p) .
\end{gathered}
$$


For every successor ordinal $\xi+1$, for every $t \in \mathbb{N}$, for every $h \in H_{t}$, we let

$$
\begin{aligned}
& \alpha_{\xi+1}(h)=\max _{a \in A} \min _{p \in P_{\xi}(h, a)} u_{t}(p), \\
& P_{\xi+1}(h)=\left\{p \in \bigcup_{a \in A} P_{\xi}(h, a): u_{t}(p) \geq \alpha_{\xi+1}(h)\right\},
\end{aligned}
$$

with the convention that the minimum over the empty set is equal to $+\infty$. For every limit ordinal $\xi$, for every $t \in \mathbb{N}$, for every history $h \in H_{t}$, we let

$$
\begin{aligned}
& P_{\xi}(h)=\bigcap_{\lambda<\xi} P_{\lambda}(h), \\
& \alpha_{\xi}(h)=\min _{p \in P_{\xi}(h)} u_{t}(p) .
\end{aligned}
$$

Proof of Theorem 3.4.1: One can show, exactly as in Flesch et al. (2010), that for each $h \in H$ the sequence $P_{\xi}(h)$ is non-increasing by inclusion and that the sequence $\alpha_{\xi}(h)$ is non-decreasing. Moreover, the game admits a subgame perfect 0 -equilibrium in pure strategies if and only if the sets $P_{\xi}(h)$ are non-empty for every ordinal $\xi$ and every history $h$. Furthermore, the set $\cap_{\xi} P_{\xi}(\varnothing)$ is exactly the set of plays that could be induced by subgame perfect 0 -equilibria of the game.

We show that $P_{\xi}(h)$ is a non-empty set for each history $h$ by induction on $\xi$. The statement is clearly true for $\xi=0$. Suppose the statement is true for the ordinal $\xi$. Consider a history $h \in H_{t}$ and let $a$ be an action that reaches the maximum in (3.16). Then $P_{\xi+1}(h) \supset P_{\xi}(h, a)$, hence $P_{\xi+1}(h)$ is non-empty, as desired.

We now turn to the more challenging case of a limit ordinal $\xi$. Suppose that $P_{\eta}(h)$ is non-empty for each $h \in H$ and each ordinal $\eta<\xi$. Let $h \in H_{t}$ be given. We recursively define a play $p=\left(h, a_{t}, a_{t+1}, \ldots\right)$. To define $a_{t}$ we distinguish two cases. 
Case 1: If $\alpha_{\eta}(h)=0$ for each $\eta<\xi$, let $a_{t}$ be any element of $A$.

Case 2: Otherwise, let $\eta<\xi$ be the least successor ordinal such that $\alpha_{\eta}(h)=1$. By (3.16), there is an action $a_{t} \in A$ such that

$$
\min _{p \in P_{\eta-1}\left(h, a_{t}\right)} u_{t}(p)=1
$$

Notice that $P_{\eta}\left(h, a_{t}\right) \subset W_{t}$.

To proceed with the definition of $p$ let $h_{t+1}=\left(h, a_{t}\right)$ and repeat the argument ad infinitum. The construction guarantees that $p \in P_{\xi}(h)$.

Grädel and Ummels (2008) consider games with qualitative objectives in a setup that is complementary to ours: there are finitely many players and each player can move infinitely many times. The authors show that the games of this class admit a subgame perfect 0-equilibrium. Unlike Grädel and Ummels (2008) who rely on Borel determinacy (Martin, 1975) to obtain their result, our technique does not require that the winning sets $W_{t}$ be Borel measurable.

\subsubsection{Games with upper semicontinuous payoffs}

The payoff function $u_{t}$ of player $t \in \mathbb{N}$ is upper semicontinuous if for every sequence $\left(p^{n}\right)_{n \in \mathbb{N}}$ of plays in $A^{\mathbb{N}}$ that converges to a limit $p \in$ $A^{\mathbb{N}}$ it holds that $\lim \sup _{n \rightarrow \infty} u_{t}\left(p^{n}\right) \leq u_{t}(p)$. Equivalently, $u_{t}$ is upper semicontinuous if for each real number $r \in \mathbb{R}$ the set $\left\{p \in A^{\mathbb{N}}: u_{t}(p) \geq\right.$ $r\}$ is closed. The game $G$ is said to have upper semicontinuous payoffs if every player $t \in \mathbb{N}$ has upper semicontinuous payoffs.

Theorem 3.4.2. If the game $G$ has upper semicontinuous payoffs, then it admits a subgame perfect 0-equilibrium in pure strategies.

Proof. As in the proof of Theorem 3.4.1, the result follows once we show that the set $P_{\xi}(h)$ as defined in Subsection 3.4.1 is a non-empty compact set for each history $h$ and each ordinal $\xi$. The proof is by induction on 

Once

$\xi$. For $\xi=0$ the statement is clearly true. Assume that for some ordinal number $\xi$ the statement is true. Take $h \in H_{t}$, and let $a \in A$ be an action that attains the maximum in (3.16). Since $P_{\xi+1}(h) \supset P_{\xi}(h, a)$, the set $P_{\xi+1}(h)$ is non-empty. Since, for every $a \in A$, the set $P_{\xi}(h, a)$ is compact by assumption and since $A$ is finite, the set $\cup_{a \in A} P_{\xi}(h, a)$ is also compact. The set $\left\{p \in A^{\mathbb{N}}: u_{t}(p) \geq \alpha_{\xi+1}(h)\right\}$ is compact because $u_{t}$ is upper semicontinuous. We conclude that $P_{\tilde{\xi}+1}(h)$ is compact.

Let $\xi$ be a limit ordinal and the statement is true for each $\lambda<\xi$. Then the statement is true for $\xi$ because the intersection of a nested family of non-empty and compact sets is non-empty and compact.

This result complements the result in Purves and Sudderth (2011), which shows that perfect information games with a finite number of players and upper semicontinuous payoff functions with finite range admit a subgame perfect 0 -equilibrium in pure strategies. Their method of proof relies on induction on the number of payoffs in the game and therefore seems difficult to extend to games with infinitely many players.

A related work is Le Roux and Pauly (2014), which considers games with bounded upper semicontinuous payoffs but does not assume that the payoff functions only take finitely many payoffs. The authors show that the game admits a 0 -equilibrium in pure strategies.

An interesting open problem is the existence of subgame perfect $\epsilon$-equilibria in games with lower semicontinuous payoffs. Due to an example in Flesch et al. (2010), we do know that such games do not always admit a subgame perfect 0 -equilibrium.

\subsubsection{Games continuous outside a countable set}

A subset $C$ of $A^{\mathbb{N}}$ is said to be co-countable if the set $A^{\mathbb{N}} \backslash C$ is countable. The game $G$ is said to be continuous outside a countable set if there exists a co-countable subset $C$ of $A^{\mathbb{N}}$ such that for each player $t \in \mathbb{N}$ the restriction of the payoff function $u_{t}$ to the set $C$, denoted by $u_{t} \mid C$, is a 
continuous function. ${ }^{3}$ The game $G$ is said to have uniformly bounded payoff functions if there exists $B<\infty$ such that, for every $t \in \mathbb{N}$, for every $p \in A^{\mathbb{N}},\left|u_{t}(p)\right|<B$. Example 3.5.2 in Section 3.5 provides an illustration of such a game.

Theorem 3.4.3. If the game $G$ has uniformly bounded payoff functions and is continuous outside a countable set, then, for every $\epsilon>0$, it has a subgame perfect $\epsilon$-equilibrium.

Proof. Let $\epsilon>0$. Let $\delta>0$ be such that $2 B M \delta<\epsilon$ and $M \delta<1$, where $M$ is the cardinality of the action set $A$. For each player $t \in \mathbb{N}$, consider a restricted strategy space $\Sigma_{t}^{\delta}$ consisting of strategies $\sigma_{t}$ such that for each $h \in H_{t}$ the probability distribution $\sigma_{t}(h)$ places a probability of at least $\delta$ on each action in $A$. Let $\Sigma^{\delta}$ be the corresponding set of strategy profiles. It is clear that for each $\sigma$ in $\Sigma^{\delta}$ the induced probability measure $\mu_{\sigma, h}$ places probability zero on each singleton set $\{p\}$ and consequently also on each countable subset of $A^{\mathbb{N}}$. In particular $\mu_{\sigma, h}$ assigns probability 0 to the complement of the set $C$. It follows that the payoff function $v_{i}(\sigma \mid h)$ is continuous on $\Sigma^{\delta}$. A standard fixed point argument can now be invoked to prove the existence of a subgame perfect 0 -equilibrium of the game with strategy spaces $\Sigma^{\delta}$. Any such strategy profile is a subgame perfect $\epsilon$-equilibrium of the original game.

Theorem 3.4.3 generalizes a result in Cingiz et al. (2016) who consider so-called centipede games played by a sequence of players.

\subsubsection{Games played by a finite number of teams}

A team is a group of players with identical payoff functions. The game $G$ is said to be played by a finite number of teams if there exists a finite

\footnotetext{
${ }^{3}$ The condition that a game is continuous outside a countable set is weaker than the condition used in Flesch and Predtetchinski (2016b).
} 
partition $\left\{T_{1}, \ldots, T_{n}\right\}$ of the player set $\mathbb{N}$ such that $u_{t}=u_{t^{\prime}}$ whenever $t, t^{\prime} \in T_{k}$ for some $k \in\{1, \ldots, n\}$.

Theorem 3.4.4. If the game $G$ is played by a finite number of teams, then it has a subgame perfect 0-equilibrium in pure strategies.

Proof. Consider a game $G^{\prime}$ where the set of players is $\{1, \ldots, n\}$, player $k^{\prime}$ s payoff function is $u_{t}$ for $t \in T_{k}$, and player $k$ makes a move at each period $t \in T_{k}$. So, the game $G^{\prime}$ has finitely many players. Moreover, as in $G$ the payoff function of each player is assumed to have a finite range, the payoff functions have finite range in $G^{\prime}$ as well. Hence, by Flesch et al. (2010) or by Bruyère et al. (2017), the game $G^{\prime}$ a strategy profile that is immune to one shot deviations, i.e. a strategy profile with the property that no player can improve his payoff at any history by deviating only once. Such a strategy profile induces a subgame perfect 0 -equilibrium of the game $G^{\prime}$.

\subsection{Two examples}

In this section we study two examples in detail. The examples are motivated by the work of Peleg (1969) and Voorneveld (2010) on minority games. The main difference here is that we look at perfect information games, whereas both Peleg and Voorneveld study games with simultaneous moves. Both examples admit a subgame perfect $\epsilon$-equilibrium. It turns out that the first example is a game with qualitative objectives, while the second game is continuous outside a countable set.

We start with a perfect information version of the game studied in Voorneveld (2010). 
Example 3.5.1. The action set is equal to $A=\{0,1\}$. For every $t \in \mathbb{N}$, player $t$ 's payoff function is given by

$$
u_{t}\left(a_{1}, a_{2}, \ldots\right)= \begin{cases}1, & \text { if } a_{t}=1 \text { and } f\left(a_{1}, a_{2}, \ldots\right) \leq 1 / 2 \\ 1, & \text { if } a_{t}=0 \text { and } f\left(a_{1}, a_{2}, \ldots\right)>1 / 2 \\ 0, & \text { otherwise }\end{cases}
$$

where

$$
f\left(a_{1}, a_{2}, \ldots\right)=\limsup _{t \rightarrow \infty} \frac{1}{t}\left(a_{1}+\cdots+a_{t}\right) .
$$

The number $f\left(a_{1}, a_{2}, \ldots\right)$ measures the frequency of action 1 in the sequence $\left(a_{1}, a_{2}, \ldots\right)$. The function $f$ is Borel-measurable. Player $t$ receives a payoff of 1 if he chooses the minority action. Otherwise player $t$ receives a payoff of 0 . More precisely, player $t$ receives a payoff of 1 if he chooses action 1 and the frequency of players choosing action 1 is less than or equal to $1 / 2$. Player $t$ also receives a payoff of 1 if he chooses action 0 and the frequency of players choosing action 1 is strictly above $1 / 2$.

As follows from Theorem 3.4.1, Example 3.5.1 has a pure strategy subgame perfect 0-equilibrium. The strategy profile $s^{\mathrm{V}}=\left(s_{t}^{\mathrm{V}}\right)_{t \in \mathbb{N}} \in S$, where

$$
\begin{aligned}
s_{1}^{\mathrm{V}}(\varnothing) & =1, \\
s_{t}^{\mathrm{V}}\left(a_{1}, a_{2}, \ldots, a_{t-1}\right) & =a_{t-1}, \quad t \in \mathbb{N} \backslash\{1\},\left(a_{1}, a_{2}, \ldots, a_{t-1}\right) \in H_{t},
\end{aligned}
$$

so player 1 plays action 1 and each player $t>1$ takes the same action as player $t-1$, is an example of such an equilibrium.

We now turn to our second example, the perfect information version of the minority game presented in Peleg (1969). 
Example 3.5.2. The action set is equal to $A=\{0,1\}$. For every $t \in \mathbb{N}$, player $t^{\prime}$ s payoff function is given by ${ }^{4}$

$$
u_{t}\left(a_{1}, a_{2}, \ldots\right)= \begin{cases}1, & \text { if } a_{t}=1, \\ 2, & \text { if } a_{t}=0 \text { and }\left(a_{1}, a_{2}, \ldots\right) \in E, \\ 0, & \text { if } a_{t}=0 \text { and }\left(a_{1}, a_{2}, \ldots\right) \in A^{\mathbb{N}} \backslash E,\end{cases}
$$

where $E$ is the set of plays such that only finitely many players take action 0 , that is

$$
E=\left\{\left(a_{1}, a_{2}, \ldots\right) \in A^{\mathbb{N}} \mid\left\{t \in \mathbb{N}: a_{t}=0\right\} \text { is finite }\right\} .
$$

Player $t$ has the option to take the safe action 1 and receive a payoff of 1 irrespective of the actions chosen by the other players. Player $t$ can also take the risky action 0 and receive a payoff of 2 when only finitely many other players choose action 0 . Otherwise, the payoff when taking the risky action is equal to 0 .

The game in Example 3.5.2 is continuous outside a countable set. To see this, let $C=A^{\mathbb{N}} \backslash E$ and note that the set $E$ is countable. For every player $t \in \mathbb{N}$, the function $u_{t} \mid C$ only depends on $a_{t}$ and is hence continuous. As follows from Theorem 3.4.3, the game admits a subgame perfect $\epsilon$-equilibrium for every positive $\epsilon$, for instance the strategy profile where each player takes action 1 with probability $1-\epsilon$ and action 0 with probability $\epsilon$. This result is tight, as the following claim shows.

Claim 3.5.3. The game of Example 3.5.2 has no subgame perfect 0-equilibrium.

Proof. Suppose that the game has a subgame perfect 0-equilibrium, say $\gamma \in \Sigma$. Let $E^{\mathrm{c}}$ be the complement of $E$, so $E^{\mathrm{c}}$ denotes the set of plays $\left(a_{1}, a_{2}, \ldots\right)$ for which the set $\left\{t \in \mathbb{N}: a_{t}=0\right\}$ is infinite. We proceed by considering two cases.

\footnotetext{
${ }^{4}$ We have added one to the payoffs in Peleg (1969) and have relabeled action 0 as action 1 and action 1 as action 0 . Clearly, this is inconsequential for the analysis of the example.
} 
CASE 1: For every $h \in H, \mu_{\gamma, h}\left(E^{\mathrm{c}}\right)=0$.

Consider a player $t \in \mathbb{N}$ and a history $h \in H_{t}$. We have $v_{t}(\gamma \mid h, 1)=1$ and $v_{t}(\gamma \mid h, 0)=2$. Since $\gamma$ is a subgame perfect 0 -equilibrium, $\gamma_{t}(h)$ puts probability 1 on action 0 . Since this is true for every player and every history, we obtain a contradiction to the assumption of case 1.

CASE 2: For some $h^{\prime} \in H, \mu_{\gamma, h^{\prime}}\left(E^{\mathrm{c}}\right)>0$.

By Lévy's zero-one law (see Appendix A), there exists a history $h \in H$ extending $h^{\prime}$ such that

$$
\mu_{\gamma, h}\left(E^{\mathrm{c}}\right)>1 / 2 \text {. }
$$

For every $k \in \mathbb{N}$, let $h^{k}=\left(h, 1^{k-1}, 0\right)$ be the history where after history $h$ first $k-1$ players take action 1 and next the last player takes action 0 . For every $k \in \mathbb{N}$, we define $F_{k}=C\left(h^{k}\right)$, the set of plays extending history $h^{k}$, so the set of plays where it takes exactly $k$ periods after history $h$ to observe action 0 . We define the singleton set of plays $F_{\infty}=\{(h, 1,1, \ldots)\}$. Observe that $\left\{F_{1}, F_{2}, \ldots, F_{\infty}\right\}$ is a partition of $C(h)$. We argue that

$$
\mu_{\gamma, h}\left(E^{\mathrm{c}} \cap F_{k}\right) \leq \frac{1}{2} \mu_{\gamma, h}\left(F_{k}\right), \quad k=1,2, \ldots, \infty .
$$

Intuitively, with respect to the measure $\mu_{\gamma, h}$, less than or equal to half of the plays in $F_{k}$ are such that the action 0 is taken infinitely many times.

Take some $k \in \mathbb{N}$. If $\mu_{\gamma, h}\left(F_{k}\right)=0$ then also $\mu_{\gamma, h}\left(E^{\mathrm{c}} \cap F_{k}\right)=0$ and inequality (3.20) holds. Let us therefore consider the case $\mu_{\gamma, h}\left(F_{k}\right)>0$. Since $F_{k}$ is equal to the cylinder set $C\left(h^{k}\right)$, we have

$$
\mu_{\gamma, h}\left(E^{\mathrm{c}} \cap F_{k}\right)=\mu_{\gamma, h^{k}}\left(E^{\mathrm{c}}\right) \cdot \mu_{\gamma, h}\left(F_{k}\right) .
$$

Let $t$ be the player who is active at history $g^{k}=\left(h, 1^{k-1}\right)$. Then player $t$ chooses action 0 with positive probability at $g^{k}$. Since $\gamma$ is a subgame perfect 0-equilibrium, it follows that $v_{t}\left(\gamma \mid g^{k}, 0\right) \geq v_{t}\left(\gamma \mid g^{k}, 1\right)=1$. It holds that

$$
v_{t}\left(\gamma \mid g^{k}, 0\right)=v_{t}\left(\gamma \mid h^{k}\right)=\mu_{\gamma, h^{k}}\left(E^{\mathrm{c}}\right) \cdot 0+\left(1-\mu_{\gamma, h^{k}}\left(E^{\mathrm{c}}\right)\right) \cdot 2,
$$


so we obtain $\mu_{\gamma, h^{k}}\left(E^{\mathrm{c}}\right) \leq 1 / 2$. Using equality (3.21), inequality (3.20) follows.

The set $E^{\mathrm{c}}$ is disjoint from $F_{\infty}$, hence $\mu_{\gamma, h}\left(E^{\mathrm{c}} \cap F_{\infty}\right)=0$, so inequality (3.20) holds.

Finally, since $\left\{F_{1}, F_{2}, \ldots, F_{\infty}\right\}$ is a partition of $C(h)$, we have

$$
\mu_{\gamma, h}\left(E^{\mathrm{c}}\right)=\mu_{\gamma, h}\left(E^{\mathrm{c}} \cap C(h)\right)=\sum_{k=1}^{\infty} \mu_{\gamma, h}\left(E^{\mathrm{c}} \cap F_{k}\right) \leq \frac{1}{2} \cdot \sum_{k=1}^{\infty} \mu_{\gamma, h}\left(F_{k}\right) \leq \frac{1}{2},
$$

obtaining a contradiction to (3.19).

As noted earlier, the result of Mertens and Neyman implies that the game of Example 3.5.2 has a 0-equilibrium. One particular 0-equilibrium of the game is as follows: all players take action 1 as long as no player has chosen action 0 . As soon as some player takes action 0 , all subsequent players take action 0 as well. This 0 -equilibrium is not subgame perfect. At a history such that some player has chosen action 0 , the 0 -equilibrium prescribes to take action 0 leading to a payoff of 0 . Switching to action 1 gives a payoff of 1 and is therefore a profitable deviation.

\subsection{Conclusion}

In this chapter, we examined perfect information games played by an infinite sequence of players, each acting only once in the course of the game. We showed that a class of so-called frequency-based minority games does not admit subgame perfect $\epsilon$-equilibrium for any $\epsilon>0$ sufficiently small.

We identified a number of sufficient conditions for the existence of subgame perfect $\epsilon$-equilibrium: games with qualitative objectives, games with upper semicontinuous payoffs, games continuous outside a countable set, and games played by a finite number of teams. 


\section{Appendix A: Lévy's zero-one law}

A general statement of Lévy's zero-one law can be found in e.g. Bogachev (2007), Example 10.3.15. Here we state a version that is sufficient for our purposes. Consider a Borel subset $P$ of $A^{\mathbb{N}}$ and let $1_{P}: A^{\mathbb{N}} \rightarrow\{0,1\}$ denote the indicator function of $P$, that is $1_{P}(p)=1$ if $p \in P$ and $1_{P}(p)=$ 0 if $p \in A^{\mathbb{N}} \backslash P$. Let $\sigma \in \Sigma$ be a strategy profile. For $t \in \mathbb{N}$, we define $\tau_{t}: A^{\mathbb{N}} \rightarrow[0,1]$ by

$$
\tau_{t}\left(a_{1}, a_{2}, \ldots\right)=\mu_{\sigma,\left(a_{1}, \ldots, a_{t}\right)}(P), \quad\left(a_{1}, a_{2}, \ldots\right) \in A^{\mathbb{N}},
$$

as the probability that the play belongs to $P$ conditional on a history of length $t$.

Theorem A.1 Let $\sigma \in \Sigma$ be a strategy profile and $h \in H$ be a history. Then the sequence $\tau_{1}, \tau_{2}, \ldots$ converges to $1_{P} \mu_{\sigma, h}$-almost surely.

According to Theorem A.1, the probability measure $\mu_{\sigma, h}$ assigns probability 1 to the set of plays $p$ for which $\tau_{t}(p)$ converges to $1_{P}(p)$. 



\section{Chapter 4}

\section{Multi-Battle $n$-Player Dynamic Contests}

\subsection{Introduction}

Many contests consist of multiple battles, where the final success or failure is determined by the outcome of these battles. For instance, to win a singles tennis match a player needs to win two or three sets before the opponent does so. ${ }^{1}$ Although sports competitions often constitute the most direct applications of multi-battle contests, such contests have already received considerable attention in other areas such as $R \& D$ races and politics as well. ${ }^{2}$ In politics, the presidential elections provide an example of simultaneous but static (i.e., not dynamic) multi-battle contests, while presidential primaries provide an example of sequential multiplebattle dynamic contests, as for each of the two major political parties

\footnotetext{
${ }^{1}$ In the PGA Tour, which brings professional male golfers together to play in a number of tournaments each year (LPGA does so for female golfers), each tournament consists of multiple battles in that golfers attempt to minimize the total number of shots they take across 72 holes.

${ }^{2}$ Harris and Vickers (1985), for instance, construed a patent race as a multi-battle contest, in which two players alternate in expending resources in a sequence of single battles. These battles or sub-contests serve as the components of the overall R\&D contest. Just like in a singles tennis match, the player who is first to win a given number of battles wins the contest, by obtaining the patent.
} 
(Democrats and Republicans) the candidate who wins the majority of the delegates across all states wins the party's nomination.

Studying a static model of resource allocation in U.S. presidential campaigns in a prominent paper, in which the goal of the candidates is to maximize their expected electoral vote, Brams and Davis (1974) highlighted the concepts of 'population of states' as well as 'population proportionality' in campaign resource allocation. ${ }^{3}$ Brams and Davis (1974, p. 113) concluded that "the winner-take-all feature of the Electoral College-i.e., that the popular-vote winner in each state wins all the electoral votes of that state-induces candidates to allocate campaign resources roughly in proportion to the $3 / 2$ 's power of the electoral votes of each state." Moreover, they show that if candidates receive delegates in proportion to their spendings on each state then population proportionality holds.

Later Lake (1979) argued that one would need to assume that the candidates maximize only their probability of winning the election, i.e., one would simply try to receive a majority of electoral votes, instead of complying with Brams and Davis' $(1973,1974)$ assumption that they maximize their expected electoral vote. Nevertheless, Lake's (1979) main result echoes Brams and Davis' (1974) impossibility of population proportionality result in that in Lake's model too it turns out that presidential candidates find it optimal to spend a disproportionately large amount of their funds in the larger states. The main difference between the two models, however, is that Brams and Davis' (1974) model, which assumes that the candidates maximize their expected electoral vote, predicts that candidates allocate campaign resources roughly in proportion to the 3/2's power of the electoral votes of each state while Lake's (1979) model,

\footnotetext{
${ }^{3}$ As noted by Brams and Davis (1974), the population of a state need not exactly reflect the proportion of the voting-age population who are registered and actually vote in a presidential election. Following Brams and Davis (1974), we too have taken the simplest course of using population as a first-approximation estimate of the proportion of voters.
} 
which assumes that the candidates maximize their probability of winning instead, predicts that candidates allocate campaign resources in proportion to the Banzhaf power index of each voter in the electorate. Nevertheless, as noted by Lake (1979, p. 130), "the Banzhaf and 3/2's rules give virtually the same results."

Sela and Erez (2013) study a two player dynamic Tullock contest. Each player maximizes the sum of the expected payoffs (similar to expected delegate maximization in our setting) for all districts. They provide a subgame prefect equilibrium such that if the winning value (number of delegates) is equal between the stages and for each resource unit that a player allocates, he loses $0 \leq \alpha \leq 1$ units of resource from his budget, then the players' resource allocations are weakly decreasing over the stages. Duffy and Matros (2015) study static contests with two players with asymmetric yet similar budgets-generalizing Lake's (1979) paper; they provide the Nash equilibrium in contests with up to four states. In a similar setting, Deck, Sarangi, and Wiser (2017) study symmetric static contests with two players. Under the assumption that players do not have budget constraints, they find the Nash equilibrium of the symmetric game (Electoral College). ${ }^{4}$

While voting occurs simultaneously in U.S. presidential elections, individuals vote sequentially in U.S. presidential primaries leading to the presidential elections, which consists of a series of elections-primariesheld across many states with different population sizes over several months.

This chapter asks the following question: Does one always have the impossibility of population proportionality in campaign resource allocation in the sequential multi-battle $n$-player dynamic-contest environment

\footnotetext{
${ }^{4}$ Additional works on dynamic resource allocation contests include the followings. Dziubinski, Goyal and Minarsch (2017) study multi-battle dynamic contests on networks in which neighboring 'kingdoms' battle in a sequential order. In a two-player and two-stage campaign resource allocation game Kovenock and Roberson (2009) characterize the unique subgame perfect equilibrium.
} 


\begin{tabular}{|l|l|}
\hline Win probability maximization & Expected delegate maximization \\
\hline Population proportionality & Population proportionality \\
does not hold (Theorem 4.3.1) & holds (Theorem 4.4.1) \\
\hline
\end{tabular}

Table 4.1: A summary of our results

of presidential primaries? We show that when players maximize their expected number of delegates (just like Brams and Davis, 1974, have assumed) there is a subgame perfect equilibrium in which players allocate their resources proportionally. However, when players maximize their probability of winning (just like Lake, 1979, has assumed), for dynamic contests with at least 4 number of states and at least 2 delegates, proportionality does not satisfy.

The chapter is organized as follows. In Section 2, we introduce a novel model for $n$-player multi-battle dynamic contests. In Section 3, we analyze the dynamic contests in which players maximize their probability of winning. We provide two examples with three and four states. In Section 4 , we show that population proportionality satisfies in dynamic contests whenever players maximize their expected number of delegates.

\subsection{Model}

We consider dynamic contests where there are $m$ states (battle fields), indexed by $t=1,2, \ldots, m$, and $n$ players, indexed by $i=1,2, \ldots, n$. The battles take place in a predetermined sequential order in states (e.g., New York, California, etc.). Each player $i$ has a fixed budget $W_{i}$ that he can allocate over the states. In each state $t$, the number of delegates that can be won is denoted by $x^{t}$. Each time period $t$, the battle at state $t$ takes place and each player $i$ simultaneously chooses a pure action (allocation) denoted by $w_{i}^{t}$ which is smaller than or equal to the budget, $W_{i}$, minus 
the already spent allocation by player $i$ till state $t$. Given the chosen actions in state $t, w^{t}:=\left(w_{1}^{t}, \ldots, w_{n}^{t}\right)$, the probability of player $i$ winning all delegates of state $t$ is defined by a contest success function

$$
p_{i}^{t}\left(w^{t}\right)= \begin{cases}\frac{w_{i}^{t}}{\sum_{j} w_{j}^{t}} & \text { if } \sum_{j} w_{j}^{t}>0 \\ \frac{1}{n} & \text { if } \sum_{j} w_{j}^{t}=0 .\end{cases}
$$

Thus, a dynamic contest is a constant sum game, as what a player wins is a loss for the other players. To avoid trivial cases, we assume that for any $t, x^{t}<\sum_{t^{\prime} \neq t} x^{t^{\prime}}$, that is, there is no "dictatorial state." Let $x_{i}^{t}$ be the number of delegates player $i$ wins at state $t$, which is $x^{t}$ with probability $p_{i}^{t}\left(w^{t}\right)$ and 0 with probability $1-p_{i}^{t}\left(w^{t}\right)$.

The set of histories of length $t$ is denoted by $H^{t}$. A history of length $t \geq 1$ is a sequence

$$
h^{t}:=\left(\left(\left(w_{1}^{1}, x_{1}^{1}\right), \ldots,\left(w_{n}^{1}, x_{n}^{1}\right)\right), \ldots,\left(\left(w_{1}^{t}, x_{1}^{t}\right), \ldots,\left(w_{n}^{t}, x_{n}^{t}\right)\right)\right)
$$

satisfying the following conditions

(i) For each $1 \leq i \leq n$ and for each $1 \leq t^{\prime} \leq t, w_{i}^{t^{\prime}} \in\left[0, W_{i}-\sum_{j<t^{\prime}} w_{i}^{j}\right]$.

(ii) For each state $t^{\prime} \leq t$, there exists a unique player $i$ such that $x_{i}^{t^{\prime}}=x^{t^{\prime}}$ and for all $j \neq i, x_{j}^{t^{\prime}}=0$.

The first property states that each action at any given state $t$ is bounded by the budget set which diminishes after each action taken in previous battles. The second property states that the winner of a battle gets all the delegates in that state (winner-takes-all). The history $H^{0}$ consists of only the empty sequence ø. Let $H=H^{0} \cup H^{1} \cup \ldots \cup H^{m}$. Note that, the history $h^{t-1}$ is presented to all players at time $t$. There is a subset $\overline{H^{t}} \subset H$ consisting of histories of length $t$ where the game comes to an end at state $t$. We call $\overline{H^{t}}$ the set of terminal histories of length $t$. If the game has not ended before state $m$ then the game ends at state $m$. We will specify terminal histories in detail later on. 
The remaining budget of player $i$ after history $h^{t} \in H$ is defined as $B_{i}\left(h^{t}\right)=W_{i}-\sum_{j \leq t} w_{i}^{j}$ where for every $j \leq t, w_{i}^{j}$ is a realized spending of history $h^{t}$. The realized winning schedule of a given history $h^{t} \in H$, denoted by $V\left(h^{t}\right)$, is the sequence of players that won the battles at states $1, \ldots, t$. Thus $V\left(h^{t}\right) \in\{1, \ldots, n\}^{t}$. For example, if $h^{3}=\left(\left(\left(w_{1}^{1}, x_{1}^{1}\right),\left(w_{2}^{1}, 0\right)\right),\left(\left(w_{1}^{2}, 0\right),\left(w_{2}^{2}, x_{2}^{2}\right)\right),\left(\left(w_{1}^{3}, 0\right),\left(w_{2}^{3}, x_{2}^{3}\right)\right)\right)$ in a twoplayer dynamic contest with $m>3$, then $V\left(h^{3}\right)=(1,2,2)$.

For player $i$, a pure strategy $\sigma_{i}$ is a sequence of $\sigma_{i}^{t}$ s such that for each $t, \sigma_{i}^{t}$ assigns, to every $h^{t-1} \in H^{t-1}$, allocation $\sigma_{i}^{t}\left(h^{t-1}\right) \in\left[0, B_{i}\left(h^{t-1}\right)\right]$. A pure strategy profile is denoted by $\sigma=\left(\sigma_{i}\right)_{i \leq n}$. The set of pure strategies of player $i \leq n$ is denoted by $\Sigma_{i}$ and the set of pure strategy profiles by $\Sigma=\times_{i \leq n} \Sigma_{i}$. For any $\sigma \in \Sigma$, let $(\sigma \mid h)=\left(\left(\sigma_{1} \mid h\right), \ldots,\left(\sigma_{n} \mid h\right)\right)$ denote the strategy profile induced by $\sigma$ in the subgame starting from history $h$.

Throughout the chapter, we will analyze two different dynamic contests in which the players either (i) maximize the probability of winning the contest, or (ii) maximize the expected number of delegates.

Maximizing probability of winning: A player wins the dynamic contest if he or she receives the plurality of delegates. In this part, we assume that players maximize the probability of winning. If at some history a player is guaranteed to lose, then the player's remaining budget after that history is 0 . Thus players who guaranteed to lose stay in the game and proportionally spend 0 at the remaining states. Furthermore, if at some history a player is already guaranteed to win, then the contest ends at this history. Accordingly, an element $\bar{h}^{t} \in H$ is called terminal if the contest ends with battle at state $t$-if either $t=m$ or there exists a player $i$ such that

$$
\sum_{j \leq t} x_{i}^{j}>\max \left\{\sum_{j \leq t} x_{i^{\prime}}^{j} \mid i^{\prime} \neq i\right\}+x^{t+1}+\ldots+x^{m} .
$$


Let $\bar{H}=\bigcup_{t \leq m} \overline{H^{t}}$ be the set of all terminal histories. For any given $\bar{h}^{t} \in \bar{H}$, let $C\left(\bar{h}^{t}\right)$ be the set of players that have won the highest number of delegates up to and including state $t$, which is defined by $C\left(\bar{h}^{t}\right)=$ $\operatorname{argmax}_{i \leq n} \sum_{j \leq t} x_{i}^{j}$. For $\bar{h}^{t} \in \bar{H}$, player $i$ receives a payoff equal to

$$
u_{i}\left(\bar{h}^{t}\right)= \begin{cases}\frac{1}{\left|C\left(\bar{h}^{t}\right)\right|} & \text { if } i \in C\left(\bar{h}^{t}\right) \\ 0 & \text { otherwise. }\end{cases}
$$

For every $t \leq m$, we define $\rho: \Sigma \times H^{t} \rightarrow \mathcal{P}(\bar{H})$ where $\mathcal{P}(\bar{H})$ is the power set of $\bar{H}$ such that $\rho\left(\sigma \mid h^{t}\right)$ denotes the set of terminal histories that are reached with positive probability with respect to $\sigma$ conditional on reaching history $h^{t} \in H^{t}$. The probability of a terminal history $\bar{h}$ being reached with respect to $\sigma$ conditional on reaching $h^{t}$ is denoted as $q\left(\sigma, \bar{h} \mid h^{t}\right)$. The payoff for player $i \leq n$ induced by a pure strategy profile $\sigma \in \Sigma$ at any history $h^{t} \in H^{t}$ is defined as

$$
v_{i}\left(\sigma \mid h^{t}\right)=\sum_{\bar{h} \in \rho\left(\sigma \mid h^{t}\right)} q\left(\sigma, \bar{h} \mid h^{t}\right) u_{i}(\bar{h})
$$

We denote $v_{i}(\sigma \mid \varnothing)$ by $v_{i}(\sigma)$. Equation $(4.5)$ and $q\left(\sigma, \bar{h} \mid h^{t}\right)$ define the payoff of each player $i$ as follows. For any given terminal history $\bar{h}$ in $\rho\left(\sigma \mid h^{t}\right)$, we multiply the probability of reaching the given history $\bar{h}$ with the utility player $i$ gets at terminal history $\bar{h}$ and then we sum over all terminal histories in $\rho\left(\sigma \mid h^{t}\right)$.

Maximizing number of delegates: Now suppose that players maximize the number of delegates they collect, so the terminal histories are exactly the histories with length $m$. The set of terminal histories is denoted by $\overline{H^{m}}$, which is equal to $H^{m}$. For any $\bar{h}^{m} \in \overline{H^{m}}$, player $i$ receives a payoff equal to

$$
\bar{u}_{i}\left(\bar{h}^{m}\right)=\sum_{t \leq m} x_{i}^{t}
$$

where $\bar{h}^{m}:=\left(\left(\left(w_{1}^{1}, x_{1}^{1}\right), \ldots,\left(w_{n}^{1}, x_{n}^{1}\right)\right), \ldots,\left(\left(w_{1}^{m}, x_{1}^{m}\right), \ldots,\left(w_{n}^{m}, x_{n}^{m}\right)\right)\right)$. 
Analogous to the maximizing probability of winning case, the set of terminal histories induced by a strategy profile $\sigma$ conditional on reaching history $h$ is denoted by $\rho(\sigma \mid h)$, which is a subset of $\overline{H^{m}}$. The payoff for player $i \leq n$ induced by a pure strategy profile $\sigma \in \Sigma$ at any $h^{t} \in H^{t}$ is

$$
v_{i}\left(\sigma \mid h^{t}\right)=\sum_{\bar{h}^{m} \in \rho\left(\sigma \mid h^{t}\right)} q\left(\sigma, \bar{h}^{m} \mid h^{t}\right) \bar{u}_{i}\left(\bar{h}^{m}\right) .
$$

Subgame perfect equilibrium: A pure strategy profile $\sigma \in \Sigma$ is a subgame perfect equilibrium if for every state $t \leq m$, for every history $h \in H^{t}$, for every player $i \leq n$, and for every strategy $\sigma_{i}^{\prime} \in \Sigma_{i}$

$$
v_{i}(\sigma \mid h) \geq v_{i}\left(\sigma_{-i}, \sigma_{i}^{\prime} \mid h\right)
$$

A strategy profile $\sigma \in \Sigma$ is a subgame perfect equilibrium if and only if for every $h \in H, \sigma$ induces an equilibrium in the subgame starting with history $h$.

Proportional strategy profile: Here we define a very specific pure strategy profile $\sigma$. For any $t$, for any non-terminal history $h^{t-1} \in H-\bar{H}$, and for any player $i$, let

$$
\sigma_{i}^{t}\left(h^{t-1}\right)=B_{i}\left(h^{t-1}\right) \frac{x^{t}}{x^{t}+\ldots+x^{m}} .
$$

We call $\sigma$ the proportional pure strategy profile. Note that under $\sigma$, no matter what the other players do, every player proportionally allocates his available budget over the remaining states.

A dynamic contest is called population proportional if the proportional strategy profile is a subgame perfect equilibrium. The focus of the chapter is the class of dynamic contests which are population proportional. 


\subsection{Maximizing the probability of winning}

In this section, the players maximize their probability of winning the primary elections-just like Lake (1979) has assumed. Here we analyze three examples of dynamic contests: i) 3-identical-state dynamic contest. ii) 4-state dynamic contest where the first state is larger (higher delegate number) than the 3 other identical states. iii) 4-state dynamic contest where the last state is larger (higher delegate number) than the first 3 identical states. We show that 3-identical-state dynamic contest satisfies population proportionality. Furthermore, we provide nontrivial examples of 4-state battles that do not admit population proportionality. The 3-state example consists of 1-delegate states while the 4-state examples involve three 1-delegate states and one 2-delegate state with two different possible spots in the sequence of battles.

Finally, we provide a non-existence result that shows for any n-player dynamic contest with at least three states, there exists a distribution of delegates over the states such that population proportionality does not satisfy. This result too echoes Brams and Davis' (1974) and Lake (1979) result on impossibility of population proportionality. We prove an extension of this result to dynamic contests.

\subsubsection{Example I: The dynamic contest with 3 identical states}

Suppose that there are two players $A$ and $B$ with equal budget $W=100$ and three identical states. We use backward induction. If for given $h^{2}$, $V\left(h^{2}\right)$ is equal to $(A, A)$ or $(B, B)$, which means player $A$ has already won or lost the first two battles, then the contest comes to an end and player $A$ wins or loses, respectively. If for given $h^{2}, V\left(h^{2}\right)$ is equal to $(A, B)$ or $(B, A)$ then the dynamic contest continues to the last battle and in the subgame after $h^{2}$, the unique best response is to allocate all of the remaining budget to the last battle. Therefore, in any subgame after any 
non-terminal history $h^{2}$ the strategy profile $\left(\sigma_{A}^{3}, \sigma_{B}^{3}\right)$, which is to spend the remaining budget to the last state, is the unique Nash equilibrium.

Now, suppose that for $h^{1}, V\left(h^{1}\right)=(A)$. Then, player $A^{\prime}$ s best response to $B$ is to maximize his payoff

$$
v_{A}\left(\sigma \mid h^{1}\right)=v_{A}\left(\left(\left(\sigma_{A}^{1}, \sigma_{B}^{1}\right),\left(\sigma_{A}^{2}, \sigma_{B}^{2}\right),\left(\sigma_{A}^{3}, \sigma_{B}^{3}\right)\right) \mid h^{1}\right)
$$

with respect to $\sigma_{A}^{2}$. Given a fixed $\sigma_{B}^{2}$, player $A$ solves the following maximization problem

$$
\begin{aligned}
& \max _{\sigma_{A}^{2}} v_{A}\left(\sigma \mid h^{1}\right)=\max _{\sigma_{A}^{2}\left(h^{1}\right)} p_{A}^{2}\left(\sigma_{A}^{2}\left(h^{1}\right), \sigma_{B}^{2}\left(h^{1}\right)\right) \\
&+\left(1-p_{A}^{2}\left(\sigma_{A}^{2}\left(h^{1}\right), \sigma_{B}^{2}\left(h^{1}\right)\right)\right) \\
&\left.\times p_{A}^{3}\left(B_{A}\left(h^{1}\right)-\sigma_{A}^{2}\left(h^{1}\right), B_{B}\left(h^{1}\right)-\sigma_{B}^{2}\left(h^{1}\right)\right)\right) \\
&=\max _{\sigma_{A}^{2}\left(h^{1}\right)} \frac{\sigma_{A}^{2}\left(h^{1}\right)}{\sigma_{A}^{2}\left(h^{1}\right)+\sigma_{B}^{2}\left(h^{1}\right)}+\frac{\sigma_{B}^{2}\left(h^{1}\right)}{\sigma_{A}^{2}\left(h^{1}\right)+\sigma_{A}^{2}\left(h^{1}\right)} \\
&\left.\times \frac{100-w_{A}^{1}-\sigma_{A}^{2}\left(h^{1}\right)}{200-w_{A}^{1}-\sigma_{A}^{2}\left(h^{1}\right)-w_{B}^{1}-\sigma_{B}^{2}\left(h^{1}\right)}\right) \\
&=\max _{w_{A}^{2}}\left(\frac{w_{A}^{2}}{w_{A}^{2}+w_{B}^{2}}+\frac{w_{B}^{2}}{w_{A}^{2}+w_{B}^{2}}\right. \\
&\left.\times \frac{100-w_{A}^{1}-w_{A}^{2}}{200-w_{A}^{1}-w_{A}^{2}-w_{B}^{1}-w_{B}^{2}}\right) .
\end{aligned}
$$

Equality (4.10) holds, since player $A$ already won the first state, in order to win the contest player $A$ either needs to win the second battle-given $\bar{h}^{2}$ with $V\left(\bar{h}^{2}\right)=(A, A)$-, or if he loses the second then to win the third battle-given $h^{2}$ with $V\left(h^{2}\right)=(A, B)$. Moreover, if the game continues to the last state, then spending the remaining budget is the unique best reponse in the subgame after given history. Equality (4.11) follows from the contest success function. Equality (4.12) follows from the definition of a strategy which maps histories to actions in the following battle.

As player $A$ wants to maximize $v_{A}\left(\sigma \mid h^{1}\right)$, player $B$ wants to minimize it. We derive best response functions from the first-order condition of 
$v_{A}\left(\sigma \mid h^{1}\right)$ with respect to $\sigma_{A}^{2}\left(h^{1}\right)$ and $\sigma_{B}^{2}\left(h^{1}\right)$, respectively. The intersection of best responses provides us the following two conditions ${ }^{5}$

$$
\begin{aligned}
w_{A}^{2} & =\frac{1}{2}\left(100-w_{A}^{1}\right), \\
w_{B}^{2} & =\frac{1}{2}\left(100-w_{B}^{1}\right) .
\end{aligned}
$$

So far, we have showed that the pair $\left(\sigma_{A}^{3}, \sigma_{B}^{3}\right)$-in which players each allocate the remaining budget to the last state-, and the pair $\left(\left(\sigma_{A}^{2}, \sigma_{B}^{2}\right),\left(\sigma_{A}^{3}\right.\right.$, $\left.\sigma_{B}^{3}\right)$ ) - in which strategies satisfy (4.13) and (4.14) —are Nash equilibria in their respective subgames. Now, we suppose that $h=\varnothing$. Then, player $A$ best responds to $B$ by maximizing his payoff $v_{A}(\sigma \mid \varnothing)=v_{A}(\sigma)$ with respect to $\sigma_{A}^{1}(\varnothing)$

$$
\begin{gathered}
\max _{\sigma_{A}^{1}(\varnothing)} v_{A}(\sigma)=\max _{\sigma_{A}^{1}(\varnothing)}\left(p_{A}^{1}\left(w^{1}\right)\left(p_{A}^{2}\left(w^{2}\right)+\left(1-p_{A}^{2}\left(w^{2}\right)\right) p_{A}^{3}\left(w^{3}\right)\right)\right. \\
\left.+\left(1-p_{A}^{1}\left(w^{1}\right)\right) p_{A}^{2}\left(w^{2}\right) p_{A}^{3}\left(w^{3}\right)\right) .
\end{gathered}
$$

where $w^{1}=\left(\sigma_{A}(\varnothing), \sigma_{B}(\varnothing)\right)$, and $w^{2}, w^{3}$ are elements of $\left[0,100-w_{A}^{1}\right] \times$ $\left[0,100-w_{B}^{1}\right]$ and $\left[0,100-w_{A}^{1}-w_{A}^{2}\right] \times\left[0,100-w_{B}^{1}-w_{B}^{2}\right]$, respectively. But since we have already concluded that whoever wins the first battle, both players should invest equally all their remaining budget to the last two states by equations (4.13) and (4.14), we can write $w_{A}^{2}=w_{A}^{3}=$ $(1 / 2)\left(100-w_{A}^{1}\right)$ and $w_{B}^{2}=w_{B}^{3}=(1 / 2)\left(100-w_{B}^{1}\right)$. Thus, we can rewrite equation (4.15) as

$$
\max _{\sigma_{A}(\varnothing)=w_{A}^{1}} \frac{\left(w_{A}^{1}-100\right)\left(\left(w_{A}^{1}\right)^{2}+3\left(w_{A}^{1}\right)\left(w_{B}^{1}-100\right)-100 w_{B}^{1}\right)}{\left(w_{A}^{1}+w_{B}^{1}-200\right)^{2}\left(w_{A}^{1}+w_{B}^{1}\right)} .
$$

Equations (4.15) and (4.16) follows from the analogous reasoning for equations $(4.10),(4.11)$ and $(4.12)$. By the same method as for $v_{A}\left(\sigma \mid h^{1}\right)$, the first order condition of $v_{A}(\sigma)$ with respect to $\sigma_{A}(\varnothing)$ and $\sigma_{B}(\varnothing)$ yields the best response functions of players $A$ and $B$. The intersection of the

\footnotetext{
${ }^{5}$ Detailed calculations are available in the Appendix.
} 
best responses leads to the condition $w_{A}^{1}=w_{B}^{1}=100 / 3$. From equations (4.13), (4.14) and $w_{A}^{1}=w_{B}^{1}=100 / 3$, we deduce that $w_{A}^{1}=w_{A}^{2}=w_{A}^{3}=$ $w_{B}^{1}=w_{B}^{2}=w_{B}^{3}=100 / 3$, which concludes that every subgame perfect equilibrium strategy profile of Example I satisfies proportionality. ${ }^{6}$

\subsubsection{Example II: Dynamic contests with 4 states}

To illustrate our model with a nontrivial example, suppose that there are two players $A$ and $B$ with equal budget $W=100$ and 4 states, one of which is a 2-delegate state and the other states each have 1 delegate. Thus, if a player wins the 2-delegate state and one other state, or wins all the 1-delegate states, then he ends up winning the dynamic contest. We next provide two examples for 4-state case in which the 2-delegate state is the first and last battle, respectively. We show that neither contest admits a proportional subgame perfect equilibrium strategy profile. ${ }^{7}$

Case $\mathbf{I}^{8}$ : Let the 2-delegate state be the first battle. If for given $h^{2}$, $V\left(h^{2}\right)$ is equal to $(A, A)$ or $(B, B)$, which means player $A$ has already won or lost the first two battles, then the contest comes to an end and player $A$ wins or loses, respectively. If for given $h^{3}, V\left(h^{3}\right)$ is equal to $(A, B, B)$ or $(B, A, A)$, then the dynamic contest continues to the last battle. And, for the subgame after the given history the unique best response for each player $\left(\sigma_{A}^{4}\right.$ and $\left.\sigma_{B}^{4}\right)$ is to allocate all the remaining budget to the last battle.

For given $h^{2}, V\left(h^{2}\right)=(A, B)$, player $A$ best responds to $B$ by maximizing his payoff $v_{A}\left(\sigma \mid h^{2}\right)$ with respect to $\sigma_{A}^{3}$

$$
\max _{\sigma_{A}^{3}} v_{A}\left(\sigma \mid h^{2}\right)=\max _{\sigma_{A}^{3}\left(h^{2}\right)} v_{A}\left(\sigma \mid h^{2}\right)=\max _{w_{A}^{3}}\left(p_{A}^{3}+\left(1-p_{A}^{3}\right) p_{A}^{4}\right),
$$

which is analogous to the dynamic contest with 3 identical states. Hence,

\footnotetext{
${ }^{6}$ This example is similar to the benchmark example of Fu et. al. (2015, p. 9); though, our settings differ when the states are not identical.

${ }^{7}$ Calculations are available upon request.

${ }^{8}$ From this point on, we take $p_{i}^{t}$ as $p_{i}^{t}\left(w^{t}\right)$.
} 
each player allocating equally his remaining budget to states 3 and 4 is the unique best response, $\left(\sigma_{A}^{3}, \sigma_{A}^{4}\right)$ and $\left(\sigma_{B}^{3}, \sigma_{B}^{4}\right)$, for subgames starting with history $h^{2}$. Now suppose that for given $h^{1}$, if $V\left(h^{1}\right)=(A)$, then player $A$ best responds to $B$ by maximizing his payoff

$$
\max _{\sigma_{A}^{2}\left(h^{1}\right)} v_{A}\left(\sigma \mid h^{1}\right)=\max _{w_{A}^{2}}\left(p_{A}^{2}+\left(1-p_{A}^{2}\right) p_{A}^{3}+\left(1-p_{A}^{2}\right)\left(1-p_{A}^{3}\right) p_{A}^{4}\right) .
$$

We derive Equation (4.17) by an analogous method from Example I. To obtain the best response functions, we take the first order conditions of the payoff functions with respect to $\sigma_{A}^{2}\left(h^{1}\right)$ and $\sigma_{B}^{2}\left(h^{1}\right)$, respectively. The intersection of the best responses yields the following two conditions

$$
\begin{aligned}
w_{A}^{2} & =\frac{1}{3}\left(100-w_{A}^{1}\right), \\
w_{B}^{2} & =\frac{1}{3}\left(100-w_{B}^{1}\right) .
\end{aligned}
$$

Given that each player wins one battle from the first two battles, we already concluded that players allocate their remaining budget to states 3 and 4 equally. Thus, by equations (4.18) and (4.19), we conclude that whoever is the winner of the first battle, players allocating equally their remaining budget to three identical states is the best response for any subgame after the first battle.

Now, we suppose that $h^{1}=\varnothing$. Then, player $A$ best responds to $B$ by maximizing his payoff $v_{A}(\sigma \mid \varnothing)=v_{A}(\sigma)$ with respect to $\sigma_{A}^{1}(\varnothing)$ which is $\max _{\sigma_{A}^{1}(\varnothing)} v_{A}(\sigma)$, i.e.,

$$
\begin{aligned}
& \max _{\sigma_{A}^{1}(\varnothing)} v_{A}(\sigma)= \max _{w_{A}^{1}}\left(p _ { A } ^ { 1 } \left(p_{A}^{2}+\left(1-p_{A}^{2}\right) p_{A}^{3}\right.\right. \\
&\left.\left.+\left(1-p_{A}^{2}\right)\left(1-p_{A}^{3}\right) p_{A}^{4}\right)+\left(1-p_{A}^{1}\right) p_{A}^{2} p_{A}^{3} p_{A}^{4}\right) \\
&=\frac{\left(w_{A}^{1}-100\right)\left(4\left(w_{A}^{1}\right)^{2}\left(w_{B}^{1}-125\right)+\left(w_{A}^{1}\right)^{3}+10000 w_{B}^{1}\right)}{\left(w_{A}^{1}+w_{B}^{1}-200\right)^{3}\left(w_{A}^{1}+w_{B}^{1}\right)} \\
&+\frac{\left(w_{A}^{1}-100\right)\left(w_{A}^{1}\left(3\left(w_{B}^{1}\right)^{2}-1100 w_{B}^{1}+70000\right)\right)}{\left(w_{A}^{1}+w_{B}^{1}-200\right)^{3}\left(w_{A}^{1}+w_{B}^{1}\right)}
\end{aligned}
$$


Equation (4.20) is derived from a similar method applied to equation (4.17). First order condition of equation (4.20) with respect to $w_{A}^{1}$ and $w_{B}^{1}$ yields the best response functions of players $A$ and $B$. We deduce that $w_{A}^{1}=w_{B}^{1}=50$ is at the intersection of the best responses. Hence, equations (4.18) and (4.19) imply that $w_{A}^{2}=w_{A}^{3}=w_{A}^{4}=w_{B}^{2}=w_{B}^{3}=$ $w_{B}^{4}=50 / 3$. In conclusion, there exists a subgame perfect equilibrium strategy profile $\sigma$ such that on an equilibrium path of $\sigma$, players spend half of their budget to the 2-delegate state and then equally split the remaining budget among 1-delegate states. Thus, case I does not admit proportionality.

Case II: Let the 2-delegate state be the last battle. If for given $h^{2}, V\left(h^{2}\right)$ is equal to $(A, B)$ or $(B, A)$, then players allocating all their remaining budget to the 2-delegate state is the unique best response for the subgame starting from history $h^{2}$. If for given $h^{2}, V\left(h^{2}\right)$ is equal to $(A, A)$, then in the following subgame of the related history, player $A$ needs only one of the remaining states and player $B$ needs both states to win the contest which is the same case as in Example I after the first battle. Thus, the unique best responses are players distributing equally their remaining budget on the last two states. Therefore, in any subgame after any history $h^{2} \in H$, there is no equilibrium that is proportional. Hence, case II does not admit proportionality.

The following theorem provides a class of dynamic contests that does not admit population proportionality whenever players maximize probability of winning.

Theorem 4.3.1. For any $m \geq 4$ number of states and any $n \geq 2$ candidates, there exists a dynamic contest, where players maximize their probability of winning, for which population proportionality fails.

Proof. Consider a dynamic contest such that $x^{1}=\ldots=x^{m-1}=1$ and $x^{m}=3$. Consider the history $h^{m-2}$ where candidate 1 wins state 1 , candidate 2 wins state 2 , candidate 1 wins state 3 and so on up to and including 
state $m-2$. Thus, for history $h^{m-2}$, the maximum amount of delegate difference between candidate 1 and candidate 2 is 1 . In the subgame after history $h^{m-2}$, candidate 1 and candidate 2 spending all their budget to the last state is the unique Nash equilibrium. Since we reach history $h^{m-2}$ with positive probability under the proportional strategy profile, the dynamic contest does not admit population proportionality.

\subsection{Maximizing the expected number of delegates}

In this section, we assume that players maximize the expected number of delegates in the $n$-player sequential multi-battle contest with the winnertake-all feature, and the following theorem provides our main result:

Theorem 4.4.1. For any dynamic contest where players maximize their expected number of delegates, population proportionality is satisfied.

Proof. We show that the proportional strategy profile $\sigma=\left(\sigma_{i}\right)_{i \leq n}$ is robust to one-shot deviations. That is, any player $i$ at any non-terminal history $h^{t}$ can not improve his payoff by changing $\sigma_{i}^{t}$, given that all other players, $j \neq i$, follow the proportional strategy. If player $i$ switches to a strategy $\bar{\sigma}_{i}=\left(\bar{\sigma}_{i}^{t+1}, \sigma_{i}^{t+2}, \ldots, \sigma_{i}^{m}\right)$ after history $h^{t}$ such that $\bar{\sigma}_{i}^{t+1}\left(h^{t}\right) \neq$ $\sigma_{i}^{t+1}\left(h^{t}\right)$, then the expected number of delegates player $i$ wins after state $t$ given the history $h^{t}$ is denoted as $v_{i, t+1}\left(\bar{\sigma}_{i}, \sigma_{-i} \mid h^{t}\right)$, which satisfies

$$
v_{i, t+1}\left(\bar{\sigma}_{i}, \sigma_{-i} \mid h^{t}\right)=\frac{x^{t+1} \bar{\sigma}_{i}^{t+1}\left(h^{t}\right)}{\bar{\sigma}_{i}^{t+1}\left(h^{t}\right)+\sum_{j \neq i} \sigma_{j}^{t+1}\left(h^{t}\right)}+v_{i, t+2}\left(\sigma \mid h_{d e v}^{t+1}\right),
$$

where $h_{\text {dev }}^{t+1}$ is a successor of $h^{t}$ with the property that at state $t+1$ player $i$ spent $\bar{\sigma}_{i}^{t+1}\left(h^{t}\right)$, and each player $j \neq i$ spent proportionally. And the expected number of delegates of player $i$ after history $h^{t}$ if she follows $\sigma$,

$$
v_{i, t+1}\left(\sigma \mid h^{t}\right)=\frac{x^{t+1} \sigma_{i}^{t+1}\left(h^{t}\right)}{\sum_{1 \leq j \leq n} \sigma_{j}^{t+1}\left(h^{t}\right)}+v_{i, t+2}\left(\sigma \mid h^{t+1}\right),
$$


where $h^{t+1}$ is a successor of $h^{t}$ with the property that at state $t+1$, each player spent proportionally. For simplicity we take

$$
\begin{gathered}
\frac{x^{t+1}+\ldots+x^{m}}{x^{t+1}}=k, \\
B_{i}\left(h^{t}\right)=a, \\
\sum_{j \neq i} B_{j}\left(h^{t}\right)=b, \\
\bar{\sigma}_{i}^{t+1}\left(h^{t}\right)=\sigma_{i}^{t+1}\left(h^{t}\right)+\Delta=\frac{a}{k}+\Delta .
\end{gathered}
$$

where $\Delta$ is a real number. We can rewrite player $i$ 's probability of winning state $t+1$ if he plays $\bar{\sigma}_{i}^{t+1}\left(h^{t}\right)$ as

$$
\frac{\bar{\sigma}_{i}^{t+1}\left(h^{t}\right)}{\bar{\sigma}_{i}^{t+1}\left(h^{t}\right)+\sum_{j \neq i} \sigma_{j}^{t+1}\left(h^{t}\right)}=\frac{\frac{a}{k}+\Delta}{\frac{a}{k}+\Delta+\frac{b}{k}}=\frac{a+\Delta k}{a+\Delta k+b},
$$

and player $i^{\prime}$ s probability of winning state $t+1$ if he plays $\sigma_{i}^{t+1}\left(h^{t}\right)$ as

$$
\frac{\sigma_{i}^{t+1}\left(h^{t}\right)}{\sum_{1 \leq j \leq n} \sigma_{j}^{t+1}\left(h^{t}\right)}=\frac{\frac{a}{k}}{\frac{a}{k}+\frac{b}{k}}=\frac{a}{a+b} .
$$

Since $\sigma$ is a proportional strategy profile, for any $t$, for any $h^{t}$, and for successor of histories where $h^{t+1}$ is a successor of $h^{t}, h^{t+2}$ is a successor of $h^{t+1}$, and so on up to and including $h^{m}$ is a successor of $h^{m-1}$, given that players follow proportional strategy profile, we have

$$
\frac{\sigma_{i}^{t+1}\left(h^{t}\right)}{\sum_{1 \leq j \leq n} \sigma_{j}^{t+1}\left(h^{t}\right)}=\frac{\sigma_{i}^{t+2}\left(h^{t+1}\right)}{\sum_{1 \leq j \leq n} \sigma_{j}^{t+2}\left(h^{t+1}\right)}=\ldots=\frac{\sigma_{i}^{m}\left(h^{m-1}\right)}{\sum_{1 \leq j \leq n} \sigma_{j}^{m}\left(h^{m-1}\right)}=\frac{a}{a+b},
$$

which means that player $i$ wins each state after $h^{t}$ with equal probability if he/she follows $\sigma_{i}$. That is, if players follow the proportional strategy profile, the proportions of the remaining budgets stay constant throughout the battles. The same property satisfies for the strategy profile $\left(\bar{\sigma}_{i}, \sigma_{-i}\right)$ after history $h_{d e v}^{t+1}$. Hence for successor of histories where $h_{d e v}^{t+2}$ is a successor of $h_{d e v}^{t+1}, h_{d e v}^{t+3}$ is a successor of $h_{d e v}^{t+2}$, and so on up to and including $h_{d e v}^{m}$ 
is a successor of $h_{d e v}^{m-1}$, given that players follow proportional strategy profile after history $h_{d e v}^{t+1}$, we have

$$
\frac{\sigma_{i}^{t+2}\left(h_{d e v}^{t+1}\right)}{\sum_{1 \leq j \leq n} \sigma_{j}^{t+2}\left(h_{d e v}^{t+1}\right)}=\frac{\sigma_{i}^{t+3}\left(h_{d e v}^{t+2}\right)}{\sum_{1 \leq j \leq n} \sigma_{j}^{t+3}\left(h_{d e v}^{t+2}\right)}=\ldots=\frac{\sigma_{i}^{m}\left(h_{d e v}^{m-1}\right)}{\sum_{1 \leq j \leq n} \sigma_{j}^{m}\left(h_{d e v}^{m-1}\right)} .
$$

Now we can simply calculate player $i$ 's probability of winning any state after history $h_{d e v}^{t+1}$, if player $i$ follows the strategy $\overline{\sigma_{i}}$

$$
\frac{\sigma_{i}^{t+2}\left(h_{d e v}^{t+1}\right)}{\sum_{1 \leq j \leq n} \sigma_{j}^{t+2}\left(h_{d e v}^{t+1}\right)}=\frac{a-\frac{a}{k}-\Delta}{a-\frac{a}{k}-\Delta+b-\frac{b}{k}} .
$$

Therefore we can rewrite equation (4.21) as

$$
v_{i, t+1}\left(\bar{\sigma}_{i}, \sigma_{-i} \mid h^{t}\right)=x^{t+1} \frac{a+\Delta k}{a+\Delta k+b}+\frac{a-\frac{a}{k}-\Delta}{a-\frac{a}{k}-\Delta+b-\frac{b}{k}}\left(x^{t+2}+\ldots+x^{m}\right),
$$

And we can rewrite equation (4.22) as

$$
v_{i, t+1}\left(\sigma \mid h^{t}\right)=\frac{a}{a+b}\left(x^{t+1}+\ldots+x^{m}\right) .
$$

We show that $v_{i}\left(\sigma \mid h^{t}\right)-v_{i}\left(\bar{\sigma}_{i}, \sigma_{-i} \mid h^{t}\right) \geq 0$, in other words show that

$$
\begin{aligned}
v_{i}\left(\sigma \mid h^{t}\right)-v_{i}\left(\bar{\sigma}_{i}, \sigma_{-i} \mid h^{t}\right)= & x^{t+1}\left(\frac{a}{a+b}-\frac{a+\Delta k}{a+\Delta k+b}\right) \\
& +\left(x^{t+2}+\ldots+x^{m}\right) \\
& \times\left(\frac{a}{a+b}-\frac{a-\frac{a}{k}-\Delta}{a-\frac{a}{k}-\Delta+b-\frac{b}{k}}\right) \geq 0 .
\end{aligned}
$$

Since $k-1=\left(x^{t+2}+\ldots+x^{m}\right) /\left(x^{t+1}\right)$, we can rewrite inequality (4.23) as

$$
\left(\frac{a}{a+b}-\frac{a+\Delta k}{a+\Delta k+b}\right)+(k-1)\left(\frac{a}{a+b}-\frac{a-\frac{a}{k}-\Delta}{a-\frac{a}{k}-\Delta+b-\frac{b}{k}}\right) \geq 0 .
$$

We can simplify inequality (4.24) as

$$
\frac{b \Delta^{2} k^{3}}{(a+b)(a(k-1)+b(k-1)-\Delta k)(a+b+\Delta k)} \geq 0 .
$$


Inequality (4.25) satisfies because we have the following conditions

$$
\begin{gathered}
a \geq \frac{a}{k}+\Delta, \\
b(k-1) \geq 0, \\
\frac{a}{k}+\Delta \geq 0 .
\end{gathered}
$$

Thus, for any $\Delta$ we have $v_{i}\left(\sigma \mid h^{t}\right)-v_{i}\left(\bar{\sigma}_{i}, \sigma_{-i} \mid h^{t}\right) \geq 0$.

Assuming that player maximize the expected number of delegates, Brams and Davis $(1973,1974)$ showed that proportionality is satisfied in their two-player static model (U.S. presidential elections). Theorem 4.4.1 extends their result to $n$-player dynamic contests.

\subsection{Conclusion}

We have shown that, when players maximize their expected number of delegates in the $n$-player sequential multi-battle contest with the winnertake-all feature, population proportionality is satisfied. On the other hand, when players simply maximize their probability of winning, population proportionality is not satisfied with at least 4 states and at least 2 candidates.

Recall that in the 2-candidate simultaneous multi-battle static setup of the U.S. presidential elections, as studied by Brams and Davis (1974) and Lake (1979), given the winner-take-all feature of elections, the impossibility of population proportionality is not affected by what candidates maximize. The main difference between Brams and Davis' (1974) modelwhich assumes that the candidates maximize their expected electoral vote-and Lake's (1979) model—which assumes that the candidates maximize their probability of winning instead-is that in the former model candidates allocate campaign resources roughly in proportion to the $3 / 2$ 's power of the electoral votes of each state while in the latter model candidates allocate campaign resources in proportion to the 
Banzhaf power index of each voter in the electorate. ${ }^{9}$ In our $n$-candidate sequential multi-battle contest model of the U.S. presidential primaries, however, population proportionality is immediately rectified once one has candidates who maximize their electoral vote instead of simply maximizing their probability of winning, despite the presence of the winner-take-all feature.

To achieve population proportionality in at least the U.S. presidential primaries which have the winner-take-all feature, a viable policy suggestion could be to provide additional incentives for candidates to induce them to win as many delegates as possible in the entire presidential primaries overall. For instance, the electoral system may provide them additional funding in the ensuing presidential race where these incentives are positively linked to the number of delegates won by the presidential candidate in the primaries. Such incentives can be very effective at the margin. As a matter of fact, even in the absence of any such additional pecuniary incentives, for one reason or another, candidates seem to already have the behavioral trait of maximizing their expected delegates themselves and do not want to stop pumping campaign funding to their remaining primaries even though they have already guaranteed winning the majority of the delegates in the primaries. The main reason that the candidates may try win more delegates beyond what they would need to guarantee their presidential candidacy (i.e., the main reason that they still might keep investing in the remaining primaries even though they know that it will not affect their chances of winning any further delegates) could be that they care about entering the U.S. presidential race with an impressive momentum gained in the presidential primaries, as Hillary Clinton tried to do against the late surge of Bernie Sanders in the U.S. Democratic primaries in 2016 even though she had already accumulated more than sufficiently many delegates to win her party's

\footnotetext{
${ }^{9}$ Further, as mentioned before, Lake (1979, p. 130) noted that the Banzhaf and 3/2's rules give virtually the same results.
} 
presidential candidacy up to that point. Nevertheless, to ensure population proportionality, the parties or the electoral system may consider boosting candidates' tendency to maximize their expected delegates via some additional pecuniary incentives, which may help at the margin at least for the candidates who may simply try to maximize their winning probability in their U.S. presidential primaries.

\section{Appendix}

\section{Example I}

The first order conditions of $v_{A}\left(\sigma \mid h^{1}\right)$ with respect to $\sigma_{A}^{2}\left(h^{1}\right)$ and $\sigma_{A}^{2}\left(h^{1}\right)$ are

$$
\begin{aligned}
\frac{\partial v_{A}\left(\sigma \mid h^{1}\right)}{\partial \sigma_{A}^{2}\left(h^{1}\right)} & =\frac{\partial v_{A}\left(\sigma \mid h^{1}\right)}{\partial w_{A}^{2}} \\
& =\frac{w_{B}^{2}\left(w_{B}^{1}+w_{B}^{2}-100\right)\left(w_{A}^{1}+2 w_{A}^{2}+w_{B}^{1}+2 w_{B}^{2}-200\right)}{\left(w_{A}^{2}+w_{B}^{2}\right)^{2}\left(w_{A}^{1}+w_{A}^{2}+w_{B}^{1}+w_{B}^{2}-200\right)^{2}}=0 .
\end{aligned}
$$

$$
\begin{aligned}
\frac{\partial v_{A}\left(\sigma \mid h^{1}\right)}{\partial \sigma_{B}^{2}\left(h^{1}\right)}= & \frac{\partial v_{A}\left(\sigma \mid h^{1}\right)}{\partial w_{B}^{2}} \\
= & -\frac{\left(w_{A}^{2}\right)^{2}\left(w_{B}^{1}+2\left(w_{B}^{2}-50\right)\right)}{\left(w_{A}^{2}+w_{B}^{2}\right)^{2}\left(w_{A}^{1}+w_{A}^{2}+w_{B}^{1}+w_{B}^{2}-200\right)^{2}} \\
& -\frac{w_{A}^{2}\left(w_{A}^{1}\left(w_{B}^{1}+2\left(w_{B}^{2}-50\right)\right)+\left(w_{B}^{1}\right)^{2}\right)}{\left(w_{A}^{2}+w_{B}^{2}\right)^{2}\left(w_{A}^{1}+w_{A}^{2}+w_{B}^{1}+w_{B}^{2}-200\right)^{2}} \\
& -\frac{w_{A}^{2}\left(2\left(w_{B}^{2}-150\right) w_{B}^{1}+2\left(w_{B}^{2}-100\right)^{2}\right)}{\left(w_{A}^{2}+w_{B}^{2}\right)^{2}\left(w_{A}^{1}+w_{A}^{2}+w_{B}^{1}+w_{B}^{2}-200\right)^{2}} \\
& -\frac{\left(w_{A}^{1}-100\right)\left(w_{B}^{2}\right)^{2}}{\left(w_{A}^{2}+w_{B}^{2}\right)^{2}\left(w_{A}^{1}+w_{A}^{2}+w_{B}^{1}+w_{B}^{2}-200\right)^{2}}=0 .
\end{aligned}
$$

From equations (4.26) and (4.27) we conclude best response functions $B R_{A}$ and $B R_{B}$ such that

$$
B R_{A}\left(\sigma_{B}^{2}\left(h^{1}\right)\right)=\frac{1}{2}\left(-w_{A}^{1}-w_{B}^{1}-2 w_{B}^{2}+200\right),
$$




$$
\begin{aligned}
B R_{B}\left(\sigma_{A}^{2}\left(h^{1}\right)\right)= & \frac{-w_{A}^{2}\left(w_{A}^{1}+w_{A}^{2}+w_{B}^{1}-200\right)}{w_{A}^{1}+2\left(w_{A}^{2}-50\right)} \\
& +\frac{\sqrt{w_{A}^{2}\left(w_{A}^{1}+w_{A}^{2}-100\right)}}{w_{A}^{1}+2\left(w_{A}^{2}-50\right)} \\
& \times \frac{\sqrt{\left(w_{A}^{2}-w_{B}^{1}+100\right)\left(w_{A}^{1}+w_{A}^{2}+w_{B}^{1}-200\right)}}{w_{A}^{1}+2\left(w_{A}^{2}-50\right)} .
\end{aligned}
$$

The intersection of best responses provides us the following two conditions

$$
\begin{aligned}
w_{A}^{2} & =\frac{1}{2}\left(100-w_{A}^{1}\right), \\
w_{B}^{2} & =\frac{1}{2}\left(100-w_{B}^{1}\right) .
\end{aligned}
$$

The first order conditions of $v_{A}(\sigma)$ with respect to $\sigma_{A}^{1}(\varnothing)$ and $\sigma_{A}^{1}(\varnothing)$ are

$$
\begin{gathered}
\frac{\partial v_{A}(\sigma)}{\partial w_{A}^{1}}=\frac{2\left(w_{B}^{1}-100\right)\left(w_{A}^{1}\right)^{2}\left(3 w_{B}^{1}-100\right)}{\left(w_{A}^{1}+w_{B}^{1}-200\right)^{3}\left(w_{A}^{1}+w_{B}^{1}\right)^{2}} \\
+\frac{2\left(w_{B}^{1}-100\right)\left(w_{A}^{1} w_{B}^{1}\left(3 w_{B}^{1}-500\right)-200\left(w_{B}^{1}-100\right) w_{B}^{1}\right)}{\left(w_{A}^{1}+w_{B}^{1}-200\right)^{3}\left(w_{A}^{1}+w_{B}^{1}\right)^{2}}=0, \\
\frac{\partial v_{A}(\sigma)}{\partial w_{B}^{1}}=-\frac{2\left(w_{A}^{1}-100\right)\left(\left(w_{A}^{1}\right)^{2}\left(3 w_{B}^{1}-200\right)\right)}{\left(w_{A}^{1}+w_{B}^{1}-200\right)^{3}\left(w_{A}^{1}+w_{B}^{1}\right)^{2}} \\
-\frac{2\left(w_{A}^{1}-100\right) w_{A}^{1}\left(3\left(w_{B}^{1}\right)^{2}-500 w_{B}^{1}+20000\right)}{\left(w_{A}^{1}+w_{B}^{1}-200\right)^{3}\left(w_{A}^{1}+w_{B}^{1}\right)^{2}} \\
+\frac{2\left(w_{A}^{1}-100\right)\left(100\left(w_{B}^{1}\right)^{2}\right)}{\left(w_{A}^{1}+w_{B}^{1}-200\right)^{3}\left(w_{A}^{1}+w_{B}^{1}\right)^{2}}=0 .
\end{gathered}
$$

From the above equations $\partial v_{A}(\sigma) / \partial w_{A}^{1}=0$ and $\partial v_{A}(\sigma) / \partial w_{B}^{1}=0$, we find the best response functions of player $A$ and $B$.

$$
\begin{aligned}
B R_{A}\left(\sigma_{B}^{1}(\varnothing)\right)= & \frac{-3\left(w_{B}^{1}\right)^{2}+500 w_{B}^{1}}{6 w_{B}^{1}-200} \\
& -\frac{\sqrt{9\left(w_{B}^{1}\right)^{3}-600\left(w_{B}^{1}\right)^{2}-70000 w_{B}^{1}+8000000} \sqrt{w_{B}^{1}}}{6 w_{B}^{1}-200} .
\end{aligned}
$$




$$
\begin{aligned}
B R_{B}\left(\sigma_{A}^{1}(\varnothing)\right)= & \frac{-3\left(w_{A}^{1}\right)^{2}+500 w_{A}^{1}}{6 w_{A}^{1}-200} . \\
& -\frac{\sqrt{9\left(w_{A}^{1}\right)^{3}-600\left(w_{A}^{1}\right)^{2}-70000 w_{A}^{1}+8000000} \sqrt{w_{A}^{1}}}{6 w_{A}^{1}-200} .
\end{aligned}
$$

The intersection of the best responses leads to the condition $w_{A}^{1}=w_{B}^{1}=$ $100 / 3$. 


\section{Bibliography}

Asheim, G. (2010). Intergenerational equity. Annual Review of Economics 2(1), 197-222.

Balbus, Ł., A. Jaśkiewicz, and A. S. Nowak (2015). Existence of stationary markov perfect equilibria in stochastic altruistic growth economies. Journal of Optimization Theory and Applications 165(1), 295-315.

Balbus, Ł., A. Jaśkiewicz, and A. S. Nowak (2015). Stochastic bequest games. Games and Economic Behavior 90, 247-256.

Bogachev, V. I. (2007). Measure Theory. Number V.II. Springer Berlin Heidelberg.

Brams, S. J. and M. D. Davis (1973). Resource-allocation models in presidential campaigning: Implications for democratic representation. Annals of the New York Academy of Sciences 219(1), 105-123.

Brams, S. J. and M. D. Davis (1974). The 3/2's rule in presidential campaigning. American Political Science Review 68(1), 113-134.

Bruyère, V. (2017). Computer aided synthesis: A game-theoretic approach. In É. Charlier, J. Leroy, and M. Rigo (Eds.), Developments in Language Theory, Cham, pp. 3-35. Springer International Publishing.

Bruyère, V., S. Le Roux, A. Pauly, and J.-F. Raskin (2017). On the existence of weak subgame perfect equilibria. In J. Esparza and A. S. Murawski 
(Eds.), Foundations of Software Science and Computation Structures, Berlin, Heidelberg, pp. 145-161. Springer Berlin Heidelberg.

Callander, S. and J. Hörner (2009). The wisdom of the minority. Journal of Economic Theory 144(4), 1421-1439.

Chatterjee, K. and T. A. Henzinger (2012). A survey of stochastic omegaregular games. Journal of Computer and System Sciences 78(2), 394-413. Games in Verification.

Cingiz, K., J. Flesch, P. J.-J. Herings, and A. Predtetchinski (2016). Doing it now, later, or never. Games and Economic Behavior 97(Supplement C), $174-185$.

De Pril, J., J. Flesch, J. Kuipers, G. Schoenmakers, and K. Vrieze (2014). Existence of Secure Equilibrium in Multi-player Games with Perfect Information, pp. 213-225. Springer Berlin Heidelberg.

Deck, C., S. Sarangi, and M. Wiser (2017). Electoral college: A multibattle contest with complementarities.

Duffy, J. and A. Matros (2015). Stochastic asymmetric blotto games: Some new results. Economics Letters 134, 4-8.

Dziubinski, M., S. Goyal, and D. E. N. Minarsch (2017). The strategy of conquest. https://doi.org/10.17863/CAM.7846.

Flesch, J., J. Kuipers, A. Mashiah-Yaakovi, G. Schoenmakers, E. Shmaya, E. Solan, and K. Vrieze (2014, November). Non-existence of subgameperfect epsilon-equilibrium in perfect information games with infinite horizon. International Journal of Game Theory 43(4), 945-951.

Flesch, J., J. Kuipers, A. Mashiah-Yaakovi, G. Schoenmakers, E. Solan, and K. Vrieze (2010). Perfect-information games with lowersemicontinuous payoffs. Mathematics of Operations Research 35(4), 742755. 
Flesch, J. and A. Predtetchinski (2016a). Subgame-perfect epsilonequilibria in perfect information games with common preferences at the limit. Mathematics of Operations Research 41(4), 1208-1221.

Flesch, J. and A. Predtetchinski (2016b, 3). Subgame-perfect epsilonequilibria in perfect information games with sigma-discrete discontinuities. Economic Theory 61(3), 479-495.

Fu, Q., J. Lu, and Y. Pan (2015). Team contests with multiple pairwise battles. The American Economic Review 105(7), 2120-2140.

Fudenberg, D. and D. Levine (1983). Subgame-perfect equilibria of finiteand infinite-horizon games. Journal of Economic Theory 31(2), 251-268.

Gabrieli, T. and S. Ghosal (2013, Jan). Non-existence of competitive equilibria with dynamically inconsistent preferences. Economic Theory 52(1), 299-313.

Goldman, S. M. (1979). Intertemporally inconsistent preferences and the rate of consumption. Econometrica 47(3), 621-626.

Gossner, O. and J. Hörner (2010). When is the lowest equilibrium payoff in a repeated game equal to the minmax payoff? Journal of Economic Theory 145(1), 63-84.

Gossner, O. and T. Tomala (2007). Secret correlation in repeated games with imperfect monitoring. Mathematics of Operations Research 32(2), 413-424.

Grädel, E. and M. Ummels (2008). Solution concepts and algorithms for infinite multiplayer games. New Perspectives on Games and Interaction 4, 151-178.

Harris, C. and J. Vickers (1985). Patent races and the persistence of monopoly. The Journal of Industrial Economics, 461-481. 
Hellwig, M., W. Leininger, P. J. Reny, and A. J. Robson (1990). Subgame perfect equilibrium in continuous games of perfect information: an elementary approach to existence and approximation by discrete games. Journal of Economic Theory 52(2), 406-422.

Herings, P. J.-J. and K. I. M. Rohde (2006). Time-inconsistent preferences in a general equilibrium model. Economic Theory 29(3), 591-619.

Jaśkiewicz, A. and A. S. Nowak (2014). Stationary markov perfect equilibria in risk sensitive stochastic overlapping generations models. Journal of Economic Theory 151, 411-447.

Kechris, A. (2012). Classical descriptive set theory, Volume 156. Springer Science \& Business Media.

Kovenock, D. and B. Roberson (2009). Is the 50-state strategy optimal? Journal of Theoretical Politics 21(2), 213-236.

Kuipers, J., J. Flesch, G. Schoenmakers, and K. Vrieze (2016). Subgameperfection in recursive perfect information games, where each player controls one state. International Journal of Game Theory 45(1-2), 205-237.

Laibson, D. (1994). Essays in hyperbolic discounting, MIT Ph. D. dissertation.

Laibson, D. (1997). Golden eggs and hyperbolic discounting. The Quarterly Journal of Economics 112(2), 443-478.

Lake, M. (1979). A New Campaign Resource Allocation Model, pp. 118-132. Heidelberg: Physica-Verlag HD.

Le Roux, S. and A. Pauly (2014). Infinite sequential games with realvalued payoffs. In Proceedings of the Joint Meeting of the Twenty-Third EACSL Annual Conference on Computer Science Logic (CSL) and the 
Twenty-Ninth Annual ACM/IEEE Symposium on Logic in Computer Science (LICS), pp. 62. ACM.

Luttmer, E. G. J. and T. Mariotti (2006, Apr). Competitive equilibrium when preferences change over time. Economic Theory 27(3), 679-690.

Mashiah-Yaakovi, A. (2008, Oct). Periodic stopping games. International Journal of Game Theory 38(2), 169.

Mertens, J.-F. (1987). Repeated games. Proceedings of the International Congress of Mathematicians, 1528-1577.

Mertens, J.-F., S. Sorin, and S. Zamir (2015). Repeated games, Volume 55. Cambridge University Press.

O'Donoghue, T. and M. Rabin (1999, March). Doing it now or later. American Economic Review 89(1), 103-124.

Peleg, B. (1969). Equilibrium points for games with infinitely many players. Journal of the London Mathematical Society 1(1), 292-294.

Peleg, B. and M. E. Yaari (1973). On the existence of a consistent course of action when tastes are changing. The Review of Economic Studies 40(3), 391-401.

Phelps, E. S. and R. A. Pollak (1968). On second-best national saving and game-equilibrium growth. The Review of Economic Studies 35(2), 185-199.

Pollak, R. A. (1968). Consistent planning. The Review of Economic Studies 35(2), 201-208.

Purves, R. A. and W. D. Sudderth (2011). Perfect information games with upper semicontinuous payoffs. Mathematics of Operations Research 36(3), 468-473. 
Radner, R. (1980). Collusive behavior in noncooperative epsilonequilibria of oligopolies with long but finite lives. Journal of economic theory 22(2), 136-154.

Renault, J., S. Scarlatti, and M. Scarsini (2008). Discounted and finitely repeated minority games with public signals. Mathematical Social Sciences 56(1), 44-74.

Renault, J., M. Scarsini, and T. Tomala (2007). A minority game with bounded recall. Mathematics of Operations Research 32(4), 873-889.

Sela, A. and E. Erez (2013). Dynamic contests with resource constraints. Social Choice and Welfare 41(4), 863-882.

Shiryaev, A. N. (1996). Probability. Second Edition, Springer-Verlag, Berlin.

Solan, E. (2000). Absorbing team games. Games and Economic Behavior 31(2), 245-261.

Solan, E. (2005). Subgame-perfection in quitting games with perfect information and differential equations. Mathematics of Operations Research 30(1), 51-72.

Solan, E. and N. Vieille (2003). Deterministic multi-player dynkin games. Journal of Mathematical Economics 39(8), 911-929.

Strotz, R. H. (1955). Myopia and inconsistency in dynamic utility maximization. The Review of Economic Studies 23(3), 165-180.

von Stengel, B. and D. Koller (1997). Team-maxmin equilibria. Games and Economic Behavior 21(1), 309-321.

von Stengel, B. and S. Zamir (2010). Leadership games with convex strategy sets. Games and Economic Behavior 69(2), 446-457. 
Voorneveld, M. (2010). The possibility of impossible stairways: Tail events and countable player sets. Games and Economic Behavior 68(1), 403-410. 



\section{Valorization}

In this chapter we discuss how we can create value from the knowledge provided in this thesis. ${ }^{10}$ This dissertation consists of three papers. The first two paper are about infinite player games, and the last paper is about infinite action multi battle $n$-player dynamic contests. These papers fall into the realm of non-cooperative game theory which analyzes individuals and self-enforced group strategies when they face a strategic interaction.

\section{Doing it now, later or never}

In this paper, we distinguish decision makers into two types, sophisticated and naive, and corresponding solution concepts sophisticated and naive equilibria. Sophisticated decision makers know how their own preferences will change in the future whereas a naive decision maker has erroneous beliefs about his/her future preferences. We show that a decision maker who faces an ambiguous due date for a task, sophisticated decision makers are more inclined to execute the task earlier than the naive ones. We achieve the ambiguous due date for a task by supposing that the game consists of infinite time periods. For example, consider a

\footnotetext{
${ }^{10}$ Article 23 of the "Regulation governing the attainment of doctoral degrees" at Maastricht University states: "Knowledge valorization refers to the 'process of creating value from knowledge, by making knowledge suitable and / or available for social (and / or economic) use and by making knowledge suitable for translation into competitive products, services, processes and new commercial activities' (adapted definition based on the National Valorization Committee 2011:8)."
} 
person who tries to quit smoking. Each day he has two choices, quitting today or quitting tomorrow. It is clear that never quitting is the worst scenario for your health so any other choice is better. Here our study suggests that the quiting probability of a sophisticated person is higher than a naive person.

Moreover to show the existence of naive and sophisticated equilibria we suppose that the payoffs are upper semi-continuous but not necessarily continuous. An example of such payoffs are the cases where stopping the game corresponds to making a costly investment, such as in the reduction of carbon dioxide emissions. Since it is too late to make investment to avoid the disastrous outcome, not making the investment at all is preferred in such cases.

\section{Perfect information games where each player acts only once}

In this paper, we study perfect information games played by an infinite sequence of players, each acting only once in the course of the game. For example, consider a game where a player can choose either 0 or 1 . Choosing 1 (safe move) leads to a payoff of 1 whereas choosing 0 (risky move) leads to a payoff of 2 if minority of the players chose that action, otherwise leads to a payoff of 0 . This game does not admit a subgame perfect $\epsilon$-equilibrium for any $\epsilon$ sufficiently small. We call this type of games as frequency-based minority game. A minority game is a type of game where players make choices sequentially and those who end up on the minority side win. Sometimes people prefer to be on the minority; such examples can be found in fashion and stock exchange transactions.

Furthermore we show that games with certain conditions admits subgame perfect $\epsilon$-equilibrium which is a stable state where there is no profitable unilateral deviations with $\epsilon>0$ error in payoffs. 


\section{Multi-battle $n$-player dynamic contests}

Primary elections are how political parties in the United States pick their strongest candidate to run for president. The parties do this by holding mini-elections in each of the states and the candidates with the most delegates from these elections become their party's official nominee. These nominees then face each other in the national election for presidency.

Winner-take-all representation rule characterizes a state election by popular vote: winner in each state wins all the electoral votes of that state. Proportional rule characterizes a state election by distributing delegates in proportion to their votes. There are eight states in primary elections that are winner-take-all, and they're all on the Republican party.

The US presidential primaries is an example of sequential multiplebattle $n$-player dynamic contests whereas presidential elections provide an example of simultaneous but static (i.e., not dynamic) multi-battle contests.

In this context, campaign resource allocation proportional to delegate numbers is desirable. We show that when players (candidates) maximize their expected number of delegates, there is an equilibrium-a stable state- in which players (candidates) allocate their resources proportionally throughout the states. However, when players maximize their probability of winning, proportionality is not satisfied for dynmaic contests with at least 4 number of states and at least 2 delegates. 



\section{Short Curriculum Vitae}

Kutay Cingiz was born in Unye, Turkey in 1986. After obtaining his Bachelor of Science degree in Mathematics at Istanbul Bilgi University in 2011, he received his Master of Arts degree in Economics at Sabanci University in 2013. He started his doctoral studies at Maastricht University Department of Economics in 2013. During his PhD, he was also a visiting scholar at Massachusetts Institute of Technology. 
